\title{
Active Flight Path Control for an Induced Spin Flight Termination System
}

\author{
Poorva J. Shukla
}

\begin{abstract}
Thesis submitted to the Faculty of the
Virginia Polytechnic Institute and State University

in partial fulfillment of the requirements for the degree of
\end{abstract}

\author{
Master of Science \\ in
}

Engineering Mechanics

Craig A. Woolsey, Chair

Muhammad R. Hajj

Nicole T. Abaid

July 25, 2017

Blacksburg, Virginia

Keywords: Linear Control Theory, Aerodynamic Flight Termination, Active Flight Path Control Copyright 2017, Poorva J. Shukla 
Active Flight Path Control for an Induced Spin Flight Termination System

Poorva J. Shukla

ABSTRACT

In this thesis, we describe a method for controlling the cycle-averaged velocity direction of a fixed-wing aircraft in an unpowered, helical descent. While the aircraft propulsion system is disabled, either intentionally or due to a failure, the aerodynamic control surfaces (aileron, elevator, and rudder) are assumed to be functional. Our approach involves two steps: (i) establishing a stable, steady, helical motion for which the control surfaces are not fully deflected and (ii) modulating the aircraft control surfaces about their nominal positions to "slant" the helical flight path in a desired direction relative to the atmosphere, whether to attain a desired impact location, to counter a steady wind, or both. The effectiveness of the control law was evaluated in numerical simulations of a general transport model (GTM). 


\section{Active Flight Path Control for an Induced Spin Flight Termination System}

Poorva J. Shukla

\section{GENERAL AUDIENCE ABSTRACT}

When an unmanned aircraft is near an authorized airspace (a region of space where the aircraft is not authorized to fly) and experiences a failure such as loss of communication with the control tower,or failure of the GPS or propulsion system, then the aircraft is generally put into an aerodynamic flight termination. In this flight termination method, the aircraft propulsion system is switched off and the control surfaces (aileron, elevator and rudder) are fixed to induce a spin in the aircraft causing it to descend in a helical fashion. However, in the presence of external gusts the aircraft might drift into the unauthorized airspace; or once the aircraft is put into spin, one may want to be able to change the impact location to a safer place. To the best of our knowledge, there exist no control strategies to alter the impact location of the aircraft once it is put into spin and while is continues to spin. In this thesis we describe a method to do so.

The aircraft impact location is altered by controlling the cycle-averaged velocity direction of a fixed-wing aircraft in an unpowered, helical descent. While the aircraft propulsion system is disabled, either intentionally or due to a failure, the aerodynamic control surfaces (aileron, elevator, and rudder) are assumed to be functional. Our approach involves two steps: (i) establishing a stable, steady, helical motion for which the control surfaces are not fully deflected and (ii) modulating the aircraft control surfaces about their nominal positions to "slant" the helical flight path in a desired direction relative to the atmosphere, whether to attain a desired impact location, to counter a steady wind, or both. The effectiveness of the control law was evaluated in numerical simulations of a general transport model (GTM). 


\section{Dedication}

To all who read it from the start to the end, or in any order, but completely. 


\section{Acknowledgments}

It has been an honor and privilege to work with my advisor, Craig Woolsey. I am thankful to him for introducing me to control theory. He did not mind when I took detours to study topics not directly related to my thesis but those that intrigued me and studying them were some of my most fun times. But I have also learnt to have discipline in my research from him. He has also been an amazing mentor, guiding me through angst when I would get stuck somewhere or start questioning the usefulness of the project.

I thank my committee members - Nicole Abaid and Muhammad Hajj for their valuable feedback. I am grateful to my department, Biomedical Engineering and Applied Mechanics, for funding me throughout my masters program. Many thanks to the various professors who taught me at Virginia Tech especially Andrew Kurdila, Jake Socha, Shane Ross and Scott Hendricks. They have not only helped me strength my concepts, but have been an inspiration in the way they are excited about their work.

I am thankful to my lab group members - Hunter, Javier, Meghan, Harsh and Jean-Mitchel for their valuable feedback and interest in my project. It was sometimes a guilty relief to know they also had bottlenecks in their work, especially when I had some too.

Special thanks to my parents, Jignasa and Jahnu, who have supported me always and encouraged me to pursue whatever interested me. 


\section{Contents}

1 Introduction $\quad 1$

1.1 Related Work . . . . . . . . . . . . . . . . . . . . 2

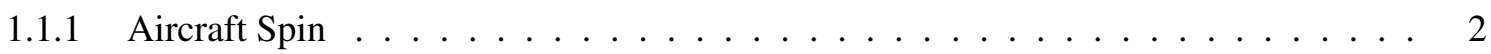

1.1.2 Bifurcation Analysis of Aircraft Dynamics $\ldots \ldots \ldots \ldots$

1.1.3 Continuation Methods $\ldots \ldots \ldots \ldots \ldots \ldots$

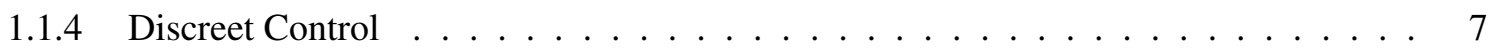

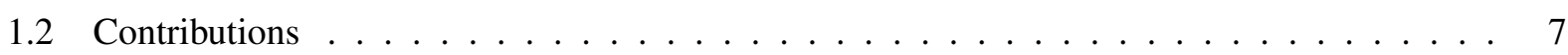

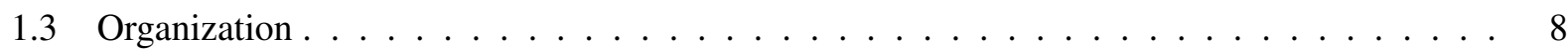

2 Problem Statement $\quad 10$

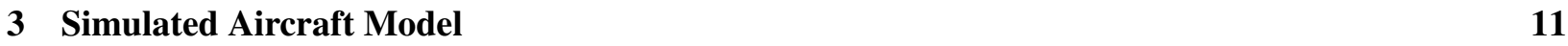

3.1 Notation . . . . . . . . . . . . . . . . . . . . . . . . 11

3.2 Equations of Motion $\ldots \ldots \ldots \ldots \ldots \ldots \ldots \ldots \ldots$

4 Nominal Solution for Spinning Descent 16

4.1 Solving for an Initial Estimate $\ldots \ldots \ldots \ldots \ldots \ldots \ldots \ldots$

4.1.1 Kinematic Angle Definitions and Assumptions . . . . . . . . . . . . . . 17

4.1 .2 Translational Dynamics $\ldots \ldots \ldots \ldots \ldots \ldots$

4.1 .3 Rotational Dynamics . . . . . . . . . . . . . . . . . . . . 22 
4.1.4 Rotational Kinematics . . . . . . . . . . . . . . . . . . . . . . . 23

4.1.5 Initial Estimate of States for a Steady Steep Spin . . . . . . . . . . . . . . . . 24

4.2 Artificial Parameter Continuation Method . . . . . . . . . . . . . . . . 25

4.3 Exact Nominal Solution . . . . . . . . . . . . . . . . . . . . . . . . . . . . . 29

4.4 Results . . . . . . . . . . . . . . . . . . . . . . . . . . 29

5 Discrete-Time Control Formulation $\quad 31$

6 Application in Simulated Flight Termination $\quad 35$

6.1 Nondimensionalization . . . . . . . . . . . . . . . . . . . . 35

6.2 Small Perturbation Equations $\ldots \ldots \ldots \ldots \ldots \ldots \ldots \ldots$

6.3 Stability of the Nominal Solution . . . . . . . . . . . . . . . . . . . 37

6.4 Region of Attraction Around the Nominal Solution . . . . . . . . . . . . . . . . 37

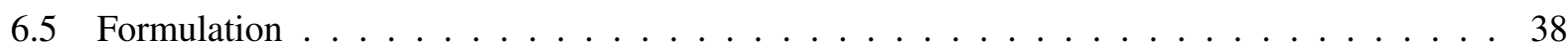

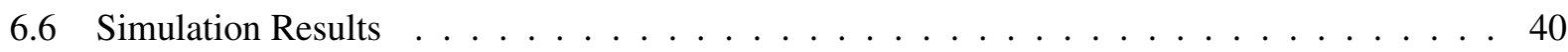

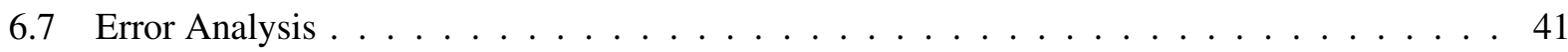

7 Conclusions and Future Work $\quad 45$

$\begin{array}{lll}\text { Appendix A } & \text { Aerodynamic Model } & 46\end{array}$

$\begin{array}{lll}\text { Appendix B Plots for Stable Equilibrium Spin Solutions } & 47\end{array}$

Appendix C Plots for Stable and Unstable Equilibrium Spin Solutions $\quad 52$

$\begin{array}{ll}\text { Bibliography } & 57\end{array}$ 


\section{List of Figures}

1.1 A schematic of spin modes based on the angle of attack. Left: Steep Spin - the angle of attack is less than $45^{\circ}$. Right: Flat Spin - the angle of attack is greater than $45^{\circ} \ldots \ldots$. . . 4

1.2 A schematic of left and right erect spins. In an erect spin, the roll and yaw rates are both in the same direction - both positive or both negative. The dotted curve represents the path behind the vertical spin axis, and the solid curve represents the path in front of the spin axis. Left: Erect Left Spin - the yaw rate is positive or pointed down. Right: Erect Right Spin -

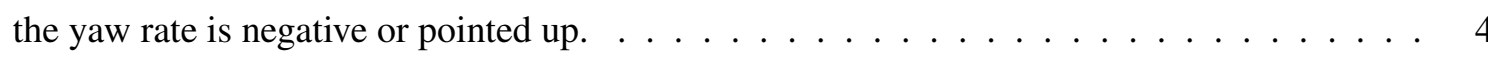

1.3 A schematic of left and right inverted spins. In an inverted spin, the roll and yaw rates are both in the opposite directions - one positive, one negative. The dotted curve represents the path behind the vertical spin axis, and the solid curve represents the path in front of the spin axis. Left: Inverted Left Spin - the yaw rate is negative or pointed up. Right: Inverted Right Spin - the yaw rate is positive or pointed down. . . . . . . . . . . 5

1.4 A schematic of the bad paths of an equilibrium curve on which conventional iterative solvers often perform poorly. . . . . . . . . . . . . . . . . . . . . . . .

1.5 Left: Vertical steady spin before the perturbed control application. Right: Slanted helix after the perturbed control application. . . . . . . . . . . . . . . . . .

3.1 A schematic diagram of the body and wind coordinate frames with respect to the aircraft. Here $\alpha$ is the angle of attack, and $\beta$ is the sideslip angle of the aircraft. . . . . . . . . . 12

3.2 A schematic diagram to show the body, wind and inertial coordinate frame transformations.

4.1 A schematic of the helical path (left) before the wing tilt and the forces (right) acting on an aircraft after the wing tilt in a steady steep spin. Adapted from [6] . . . . . . . . . . . 18

4.2 A schematic of the kinematic angle $\chi$ (left) the resulting wing tilt $\theta_{y}$ (right) with respect to the horizontal plane. Adapted from [49] and [6] . . . . . . . . . . . . . . . . . . . 


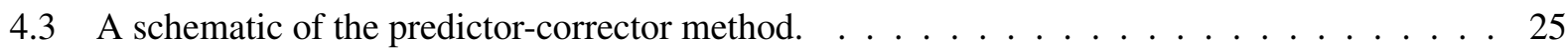

4.4 Equilibrium spin solutions for $-15<\delta_{a}<0,10<\delta_{e}<30,-30<\delta_{r}<30$. Yellow circles are stable equilibria and blue circles are unstable equilibria. The black cross is the nominal solution chosen for the control application in chapter $6 \ldots \ldots \ldots$. . . . . . . . . . 30

6.1 Estimation of the region of attraction: Monte-Carlo simulation results, when a maximum of $40 \%$ perturbation is applied to the state variable about the reference spin equilibrium. The solid black line is the nondimensional nominal state and the dashed black line is at a $10 \%$ perturbation from the nominal value. . . . . . . . . . . . . . . . . . . 39

6.2 Schematic to show $\boldsymbol{\varepsilon}_{d} \ldots \ldots \ldots \ldots \ldots \ldots \ldots \ldots \ldots \ldots$

6.3 The nominal trajectory of an aircraft in a steep vertical spin is shown in black. The controlled trajectory of an aircraft to direct it along $\theta_{1 \mathrm{~d}}=10^{\circ}$ and $\theta_{2 \mathrm{~d}}=0.1^{\circ}$ is demonstrated by a nonlinear model in blue and by a linear model in red. . . . . . . . . . . . . . . . .

6.4 Variation of the achieved inclination angle $\lambda_{1 \mathrm{~g}}$ with varying desired inclination angles $\lambda_{1 \mathrm{w}}$ and desired azimuth angles $\lambda_{2 \mathrm{w}}$. Here the time interval for which the constant control is applied $\Delta T$ is $\frac{1}{5}$. The dashed black line is a slope 1 line for reference. . . . . . . . . . . .

6.5 Variation of the achieved inclination angle $\lambda_{1 \mathrm{~g}}$ with varying desired inclination angles $\lambda_{1 \mathrm{w}}$ and desired azimuth angles $\lambda_{2 \mathrm{w}}$. Here the time interval for which the constant control is applied $\Delta T$ is $\frac{1}{10}$. The dashed black line is a slope 1 line for reference. . . . . . . . .

6.6 Variation of the achieved inclination angle $\lambda_{1 \mathrm{~g}}$ with varying desired inclination angles $\lambda_{1 \mathrm{w}}$ and desired azimuth angles $\lambda_{2 \mathrm{w}}$. Here the time interval for which the constant control is applied $\Delta T$ is $\frac{1}{15}$. The dashed black line is a slope 1 line for reference. . . . . . . . .

6.7 Variation of the achieved azimuth angle $\lambda_{2 \mathrm{~g}}$ with varying desired inclination angles $\lambda_{1 \mathrm{w}}$ and desired azimuth angles $\lambda_{2 \mathrm{w}}$. Here the time interval for which the constant control is applied $\Delta T$ is $\frac{1}{5}$. The dashed black line is a slope 1 line for reference. . . . . . . . . .

6.8 Variation of the achieved azimuth angle $\lambda_{2 \mathrm{~g}}$ with varying desired inclination angles $\lambda_{1 \mathrm{w}}$ and desired azimuth angles $\lambda_{2 \mathrm{w}}$. Here the time interval for which the constant control is applied $\Delta T$ is $\frac{1}{10}$. The dashed black line is a slope 1 line for reference. . . . . . . . . .

6.9 Variation of the achieved azimuth angle $\lambda_{2 \mathrm{~g}}$ with varying desired inclination angles $\lambda_{1 \mathrm{w}}$ and desired azimuth angles $\lambda_{2 \mathrm{w}}$. Here the time interval for which the constant control is applied $\Delta T$ is $\frac{1}{15}$. The dashed black line is a slope 1 line for reference. . . . . . . . . . . 
B.1 Relation between the stability of the steady spin equilibria and the control inputs. The solutions are for the radius of the helix varying from $1-5 \mathrm{~m}$. Red circles: unstable equilibria,

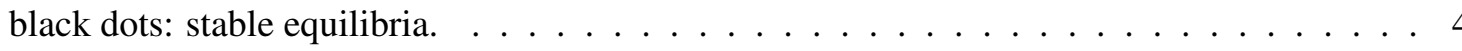

B.2 Variation of $\phi$ and $\theta$ at stable spin equilibria, with the control inputs $\delta_{a}, \delta_{e}$ and $\delta_{r}$. The equilibria are restricted to the radius of the helix varying from $1-5 \mathrm{~m} \ldots \ldots \ldots \ldots$

B.3 Variation of the body velocities $u, v$ and $w$ at the stable spin equilibria, with the control inputs $\delta_{a}, \delta_{e}$ and $\delta_{r}$. The equilibria are restricted to the radius of the helix varying from $1-5 \mathrm{~m}$. . .

B.4 Variation of the body angular velocities $p, q$ and $r$ at the stable spin equilibria, with the control inputs $\delta_{a}, \delta_{e}$ and $\delta_{r}$. The equilibria are restricted to the radius of the helix varying

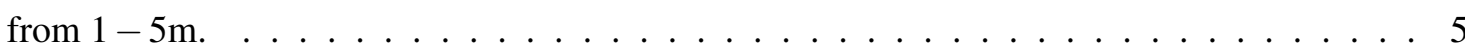

B.5 Variation of the angular speed (which is equal to the yaw rate at equilibrium) $\omega$, the linear speed $V$ and the 2-norm of the eigenvalues $\|\lambda\|_{2}$ with the control inputs $\delta_{a}, \delta_{e}$ and $\delta_{r}$. The equilibria are restricted to the radius of the helix varying from $1-5 \mathrm{~m} \ldots \ldots \ldots \ldots \ldots$

C.1 Relation between the stability of the steady spin equilibria and the control inputs. Red circles: unstable equilibria, black dots: stable equilibria. The radius of the helical descent of the equilibria is restricted to $200 \mathrm{~m} \ldots \ldots \ldots \ldots \ldots \ldots$

C.2 Variation of $\phi$ and $\theta$ at stable spin equilibria, with the control inputs $\delta_{a}, \delta_{e}$ and $\delta_{r}$. The radius of the helical descent of the equilibria is restricted to $200 \mathrm{~m} \ldots \ldots \ldots \ldots$

C.3 Variation of the body velocities $u, v$ and $w$ at the stable spin equilibria, with the control inputs $\delta_{a}, \delta_{e}$ and $\delta_{r}$. The radius of the helical descent of the equilibria is restricted to $200 \mathrm{~m}$. . . . 5

C.4 Variation of the body angular velocities $p, q$ and $r$ at the stable spin equilibria, with the control inputs $\delta_{a}, \delta_{e}$ and $\delta_{r}$. The radius of the helical descent of the equilibria is restricted to

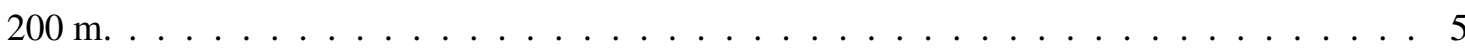

C.5 Variation of the angular speed (which is equal to the yaw rate at equilibrium) $\omega$, the linear speed $V$ and the 2-norm of the eigenvalues $\|\lambda\|_{2}$ with the control inputs $\delta_{a}, \delta_{e}$ and $\delta_{r}$. The radius of the helical descent of the equilibria is restricted to $200 \mathrm{~m} \ldots \ldots \ldots \ldots$ 


\section{List of Tables}

1.1 Spin modes classified by NASA, from $[11] \ldots \ldots \ldots \ldots$ 


\section{Chapter 1}

\section{Introduction}

In a survey of emergency recovery and flight termination (ERFT) systems, Stansbury et al [46] noted that the safe operation of unmanned aircraft systems (UAS) in public airspace requires ...

... technologies and flight procedures to ensure that neither people nor property ... are endangered by the failure of an on-board component, by inappropriate unmanned aircraft (UA) response to pilot commands, or by inadvertent entry by the UA into prohibited airspace. The aircraft must be equipped with emergency recovery (ER) procedures and technologies that ensure that in the event of such a failure that the UA is recovered intact with minimal risk to other aircraft, people, or property. Finally, should ER procedures prove ineffective and it is impossible to recover the UA, the pilot-in-command and/or the UAS may engage flight termination (FT) procedures-activities to ensure that the UA is safely destroyed (should the UA be so equipped) or immediately grounded.

The authors describe the FT protocols offered by a number of commercial autopilots and the FT systems that are integrated into several commercial UAS. One commonly used FT protocol is "aerodynamic termination" in which the malfunctioning aircraft, if able, executes a control sequence that will intentionally crash the aircraft "in a somewhat controlled manner" [46]. For a fixed-wing UAS, the control sequence might establish a gliding descent, a slow downward spiral, or a steep spin.

Aerodynamic flight termination using spin is ideal when the UAS is experiencing link loss (LL), GPS loss, has a failure in the propulsion system or has low fuel, and is near an unauthorized airspace. The nominal, helical descent, which is nearly vertical, prevents deeper intrusion into the unauthorized airspace. This high-drag, rotating motion effectively dissipates kinetic energy also. This type of flight termination protocol was successfully, if inadvertently, demonstrated during a March 29, 1999 flight test of the Northrop Grumman Global Hawk [33]. A similar FT system is used by the Insitu Scan Eagle, the smaller scale NASA 
Airborne Subscale Transport Aircraft Research (AirSTAR) UAS [14], and a number of autopilots including the Cloudcap Piccolo [48], Micropilot autopilots [27], and the Kestrel autopilots [25]. The failsafe is even mandated by the AIAA for entries in its popular annual Student Design/Build/Fly Competition [4]

In this thesis, we develop a method to "slant" the helical flight path in a desired direction relative to the atmosphere, whether to attain a desired impact location, to counter a steady wind, or both. The effectiveness of the control law was evaluated in numerical simulations of a general transport model (GTM).

In fact the control strategy developed is general and can be used to steer the averaged output of any periodic system, like flapping flight, walking, running etc.

\subsection{Related Work}

Our method involves two steps: (i) establishing a stable, steady, helical motion for which the control surfaces are not fully deflected and (ii) modulating the aircraft control surfaces about their nominal positions to control the cycle-averaged velocity direction of a fixed-wing aircraft in an unpowered, helical descent. With that context, previous work on computing and analyzing an aircraft spin, and related control strategies are discussed in the rest of the chapter.

\subsubsection{Aircraft Spin}

Spins are steady or oscillatory states with a non-zero angular rate. Entry into a spin typically occurs by a jump phenomenon due to the onset of an instability on a coexisting low- $\alpha$ branch of steady states [22]. Generally it is due to a stall instability from a level flight trim condition, in which case the spin is called a stall-spin.

In a stall-spin, an aircraft has atleast one of its wings stalled, or stalled deeper than the other, leading to non-zero angular rates. In this case, the resulting angle of attack on the two wings is different. This generates unequal lift and drag forces on the wings. The asymmetric lift tends to roll the aircraft, while the asymmetric drag tends to yaw the aircraft. In most aircraft, while all the rotary damping coefficients are stable at low angles of attack, some of the coefficients (mostly the roll damping coefficient or $C_{l}$ ) changes sign at high angles of attack near stall, becomes unstable and promotes spin. Thus once a roll is initiated, the airplane has an autorotative tendency that builds up the roll moment. Near stall angles, the ailerons lose their effectivity or result in a control reversal (a right aileron control leads to a left roll and vise-versa). So the rolling moment cannot be stopped by the aileron control alone. A detailed explanation can be found in any standard atmospheric flight book, like [19] and [6]. The airplane's inertial roll-yaw coupling develops a yawing motion. Near stall, there is a drop in the overall lift produced by the wings and the airplane nose drops. This combined roll-pitch-yaw motion of a stalled aircraft is called post-stall gyration. Once the yaw 


\begin{tabular}{|l||c|l|c|l|}
\hline spin mode & steep & $\begin{array}{l}\text { moderately } \\
\text { steep }\end{array}$ & $\begin{array}{l}\text { moderately } \\
\text { flat }\end{array}$ & flat \\
\hline angle of attack & $20^{\circ}-30^{\circ}$ & $30^{\circ}-45^{\circ}$ & $45^{\circ}-65^{\circ}$ & $65^{\circ}-90^{\circ}$ \\
\hline nose attitude & \multicolumn{2}{|c|}{ extreme nose-down } & \multicolumn{2}{|c|}{ less nose-down } \\
\hline rate of descent & \multicolumn{2}{|c|}{ very rapid } & less rapid \\
\hline rate of roll & extreme & moderate \\
\hline yaw rate & moderate & extreme \\
\hline $\begin{array}{l}\text { wingtip to wingtip difference in angle } \\
\text { of attack }\end{array}$ & modest & large \\
\hline nose to tail difference in slip & large & large \\
\hline
\end{tabular}

Table 1.1: Spin modes classified by NASA, from [11].

rate builds up, the relative air-speed on the wings increase, and the nose rises. The flight path steepens, and the aircraft starts losing height. The airplane is now said to be in a spin, and descends vertically downwards in a more or less helical path.

It is difficult to recover from a fully developed stable spin. In fact there have been several aircraft crashes in the 1900s due to stall/spin. Most of the early aircrafts flew at low altitudes, so if a stall-spin departure occured, the aircraft crashed before the spin was completely developed, making it difficult to analyze the cause. During the seven year period 1965-72, an average of two aircrafts per month were lost by the US Navy and the Marine Corps together. See [18], [3], [1] and the references therein for a historical review of spin. In the 1970s NASA conducted a series of experiments on the spin characteristics of the general aviation aircrafts ([12], [11]) and developed a classification of the spin modes based on the angle of attack as described in table 1.1 .

Unlike a level flight, spin is difficult to study via theoretical analysis because of the nonlinear, inertial cross coupling between the longitudinal and lateral degrees of freedom. The aerodynamics during the transition to spin are also extremely complex because of the extensive flow separation over the wing and tail surfaces. Therefore, most of the early spin analysis was done experimentally (eg. see [9] and [26]). However, it is possible to study developed or steady-state spin via theoretical analysis. Early attemps to theoretically study spin used approximate methods or reduced order models to predict equilibrium spin states and control combinations ([47] and [8] are good examples). These were mainly based on force and moment equilibrium under steady conditions, for small sideslip angles $\beta$ and pitch-rates $\dot{q}$. Detailed pictures representing the kinetics and angles can be found in [49] and similar schematic diagrams are included in this thesis in chapter 4. One of these approximate methods is implemented in chapter 4 to obtain an initial guess for a steady spin state, which is fed to a continuation method to obtain a manifold of equilibria with varying control inputs. Another analytical criteria for aircraft spin susceptibility, based on the fact that the entry into a steady steepspin mode occurs through a saddle node bifurcation, is given by [42].

The introduction of bifurcation methods and continuation algorithms has made it possible to numerically 


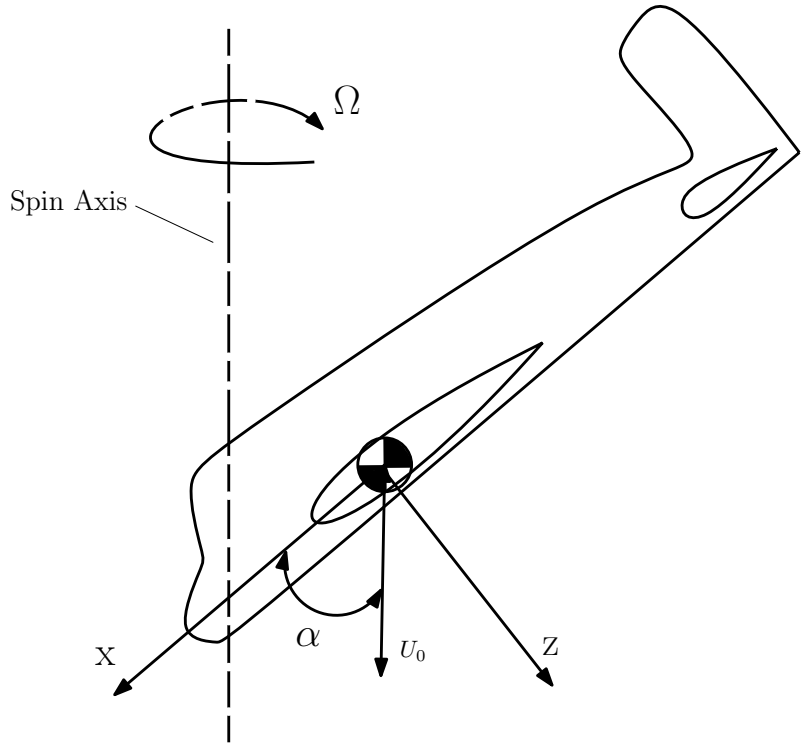

(a) Steep Spin

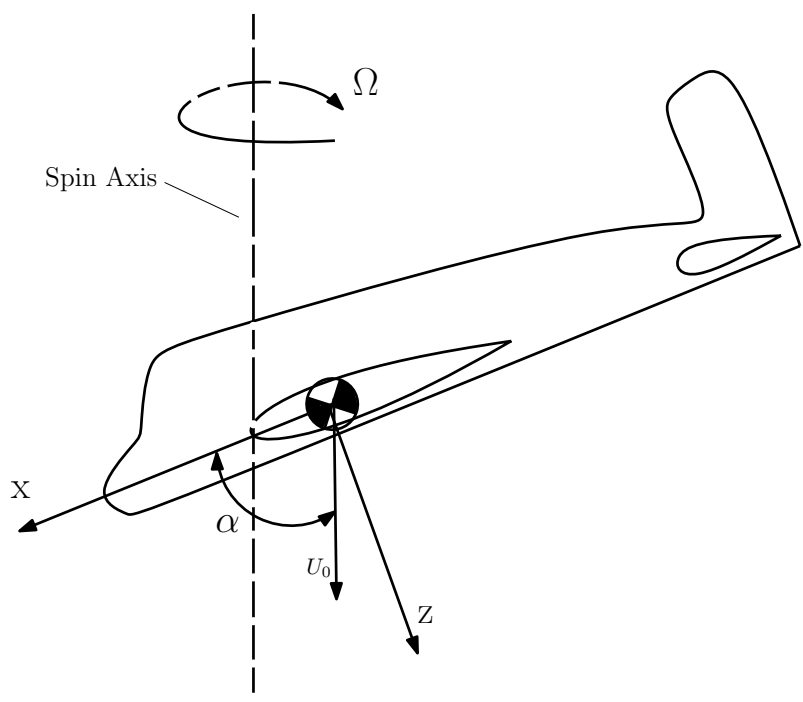

(b) Flat Spin

Figure 1.1: A schematic of spin modes based on the angle of attack. Left: Steep Spin - the angle of attack is less than $45^{\circ}$. Right: Flat Spin - the angle of attack is greater than $45^{\circ}$.

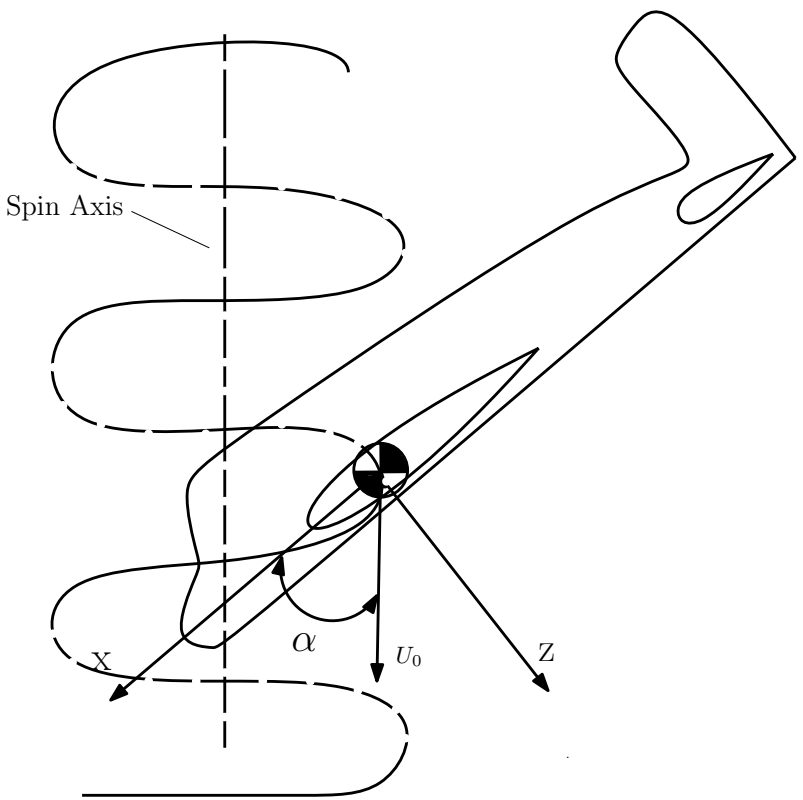

(a) Erect Left Spin

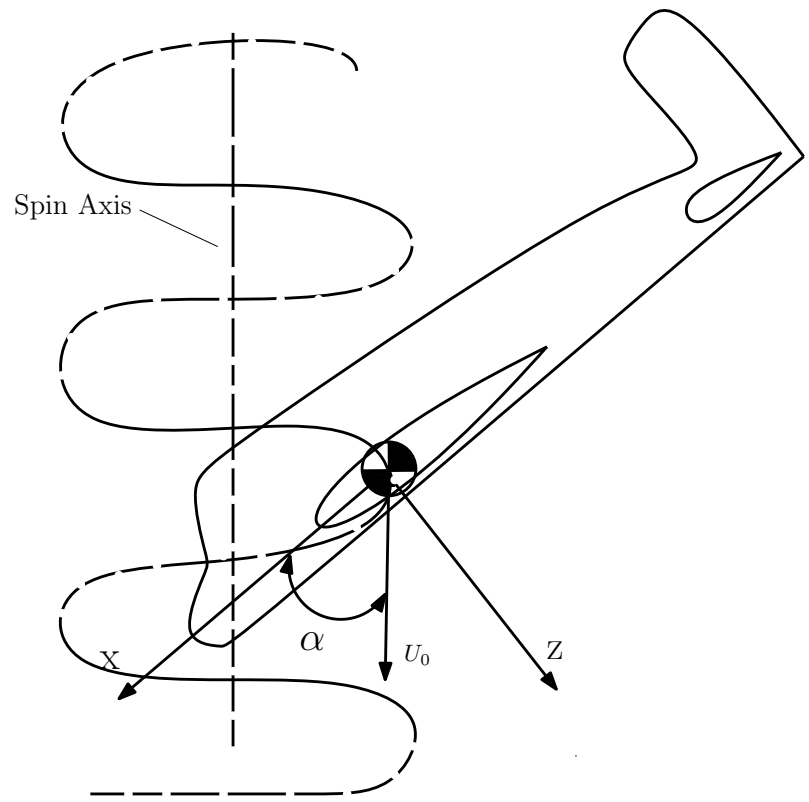

(b) Erect Right Spin

Figure 1.2: A schematic of left and right erect spins. In an erect spin, the roll and yaw rates are both in the same direction - both positive or both negative. The dotted curve represents the path behind the vertical spin axis, and the solid curve represents the path in front of the spin axis. Left: Erect Left Spin - the yaw rate is positive or pointed down. Right: Erect Right Spin - the yaw rate is negative or pointed up. 


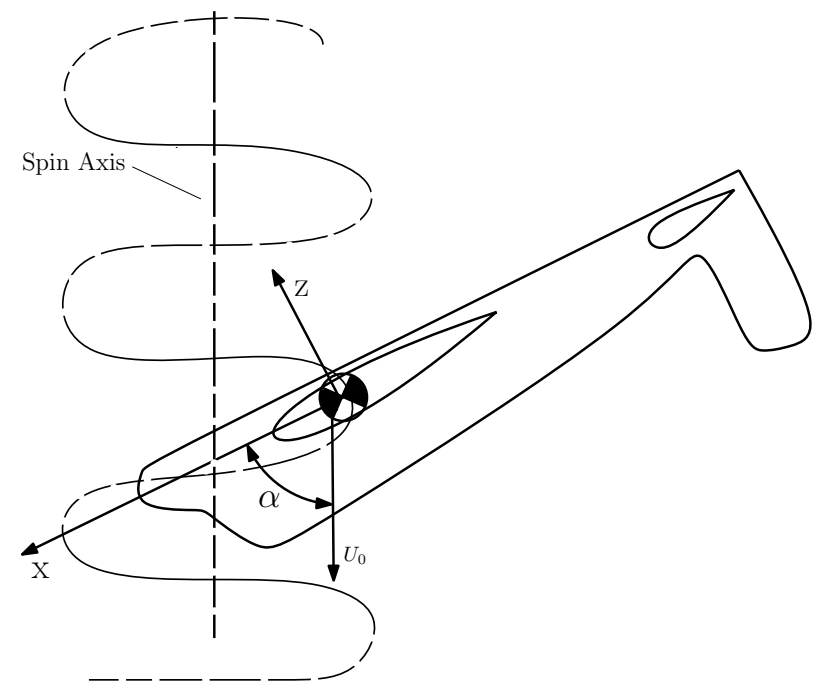

(a) Inverted Left Spin

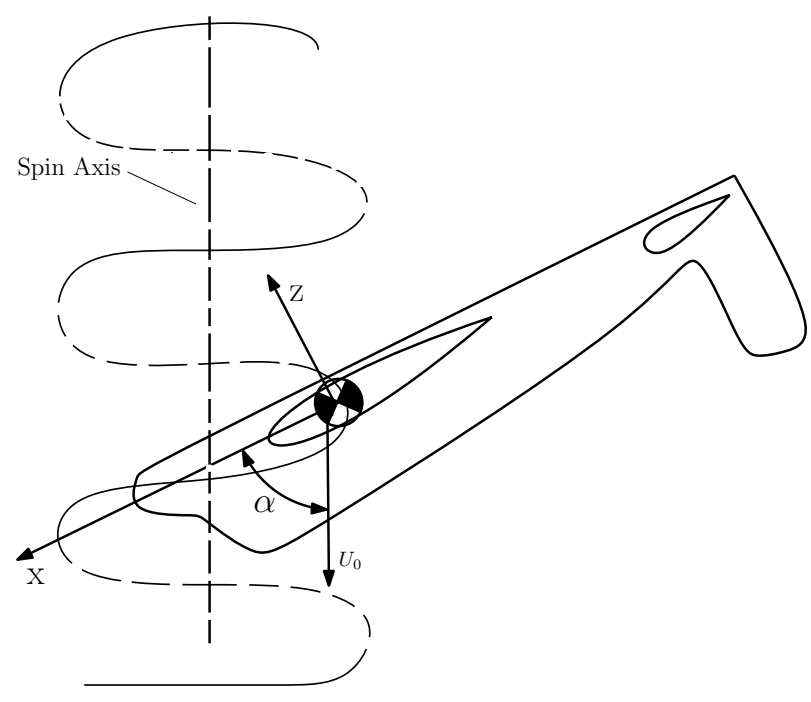

(b) Inverted Right Spin

Figure 1.3: A schematic of left and right inverted spins. In an inverted spin, the roll and yaw rates are both in the opposite directions - one positive, one negative. The dotted curve represents the path behind the vertical spin axis, and the solid curve represents the path in front of the spin axis. Left: Inverted Left Spin - the yaw rate is negative or pointed up. Right: Inverted Right Spin - the yaw rate is positive or pointed down.

compute the complete set of spin solutions for a given aircraft without any approximations (eg. [21], [13] and [43]). The spin solutions can be steady equilibrium points (resulting in a uniform vertical helical trajectory) or limit cycles (resulting in oscillatory spins), stable or unstable, erect (roll and yaw in the same direction) or inverted (roll and yaw in the opposite directions), flat or steep (depending on the angle of attack), right or left handed (depending on the yaw rate direction). Refer to figures 1.1 - 1.3 for schematic diagrams. The nature of the spin can be predicted from a bifurcation diagram.

\subsubsection{Bifurcation Analysis of Aircraft Dynamics}

The macroscopic motion of an aircraft is defined by a set of coupled nonlinear ordinary differential equations (ODEs) of the form:

$$
\dot{x}=f(x, u)
$$

Here $\boldsymbol{x}$ is a vector of the state variables and $\boldsymbol{u}$ is a vector of the control inputs. In special situations, the equations are linearized about a reference condition, making it possible to study small perturbations about the reference condition by a set of linear ODEs. However, in this thesis, in order to choose a stable steady spin state with a relatively large region of attraction, the global spin characteristics of the aircraft is studied; so linearization would not work. Techniques from dynamical systems theory, specifically a bifurcation analysis, can be applied to study the global characteristics of the nonlinear ODEs. 
Bifurcation analysis helps to determine if and when the qualitative nature of the solutions change with a change in the system parameters, here the control inputs. Mehra, Kessel and Carol [38] were among the first to suggest a dynamical systems approach to achieve a global qualitative understanding of nonlinear aircraft dynamics. Since then, a bifurcation analysis has been used several times to study various aspects of flight regimes ([28], [21], [2]) and it is now a well-established approach. While the earlier works were devoted mainly to the theoretical methods and associated numerical procedures which can be used in aircraft dynamics, the later publications more often present the results applied to real aircraft ([37], [5], [20] and the references there in).

Bifurcation analysis has been previously applied to the GTM and among other flight regimes, spin was also studied [39]. A rigorous assessment of the ability to control and regulate an aircraft around bifurcations is provided in [17] and [36], with a focus on loss of control. They define safe sets in which the aircraft can be positively controlled by studying the controllability and observalibility of a closed-loop GTM model. We apply a similar procedure to identify the spin equilibria where the aircraft can be controlled in order to slant the helical descent in a desired direction.

\subsubsection{Continuation Methods}

The qualitative analysis of any system of ODEs involves solving for the equilibrium curves and/or surfaces for the given dynamical system and identifying the bifurcations. A continuation method is most often used to contruct the equilibrium curves. D.F. Davidenko's seminal work [15] formed the foundations of most contemporary continuation methods, though the method has evolved considerably since then. R. W. Klopfenstein [34] and H.B. Keller [30] have greatly refined the method to bring it to its present sophistication. The foundations of the continuation method and details about its variations are summarized in [13].

In the recent past, continuation methods have gained a lot of popularity because of their significant advantages over conventional alternatives (such as iterative nonlinear solvers like the Newton-Raphson method). Although Newton's method is a rapidly convergent scheme, it requires a good initial approximation and finds only one solution (not a curve of solutions). Certain variations of continuation methods can find all solutions without an initial guess. Conventional iterative solvers often perform poorly when the equilibrium path is steep, split, divergent or backtracking (see Fig. 1.4). An artificial parameter continuation method overcomes all of these problems.

An aircraft model is high dimensional $\left(\boldsymbol{x} \in \mathbb{R}^{8}\right)$ and with the coupled nonlinearities the equilibrium paths can be fairly complex (eg. see [41]). Therefore in this thesis, an artificial parameter continuation method based on pseudo arclength is used to compute the equilibrium paths. 


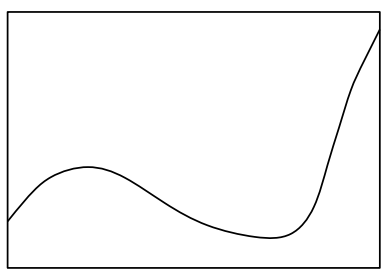

(a) Steep

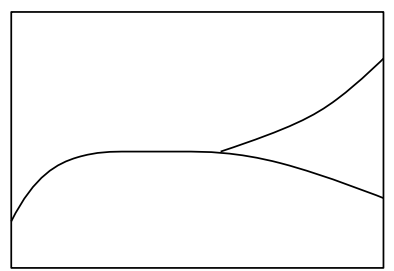

(b) Split

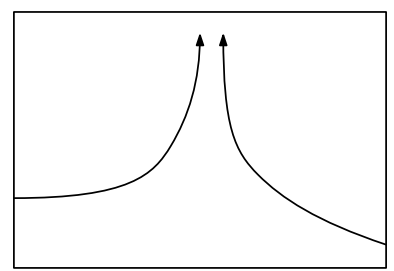

(c) Divergent

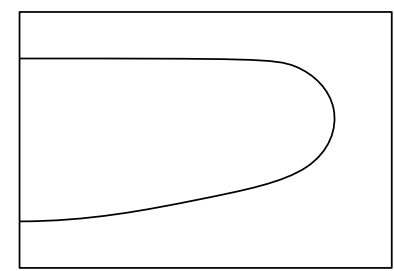

(d) Backtracking

Figure 1.4: A schematic of the bad paths of an equilibrium curve on which conventional iterative solvers often perform poorly.

\subsubsection{Discreet Control}

The proposed algorithm involves applying control deviations during a fixed fraction of each helical rotation. The approach to control is reminiscent of flapping-wing micro-air vehicle (FWMAV) control strategies that operate over a fraction of a nominal flapping cycle [16, 7]. Assuming the nominal motion is sufficiently stable, one expects the transient effect of small perturbations caused by modulating the control surfaces to decay after the surfaces return to their nominal state. It is important to note, however, that the spinning equilibrium is just one of many solutions for the nonlinear equations of motion of an unpowered aircraft; a sufficiently large excursion could induce an entirely different stable motion, such as a flat spin, a phenomenon well-known to aerobatic pilots [32]. I demonstrate the feasibility of the proposed strategy in simulation, but true validation would require an experimental flight test program.

\subsection{Contributions}

In this thesis, I describe an approach to control the direction of the helical descent by a fixed-wing aircraft in a steep spin. In this approach, the aircraft propulsion system is disabled, whether intentionally or due to a failure, but the aircraft retains control of the aerodynamic surfaces (the aileron, elevator, and rudder). Note that this assumption is implicit in the use of an aerodynamic FT system. It is also assumed that the UAS retains navigation and control functionality (i.e., motion sensing and control computation) required to implement the procedure we describe.

Our approach involves two steps: (i) establishing a stable, steady, helical motion for which the control surfaces are not fully deflected and (ii) modulating the aircraft control surfaces about their nominal positions to "slant" the helical flight path in a desired direction relative to the atmosphere, whether to attain a desired impact location, to counter a steady wind, or both. As illustrated by simulations of the AirSTAR UAS FT system, a steady wind can significantly affect the impact location for an aircraft at altitude [14].

Following is an outline of the approach used to formulate a vertical spin solution and to develop a flight 


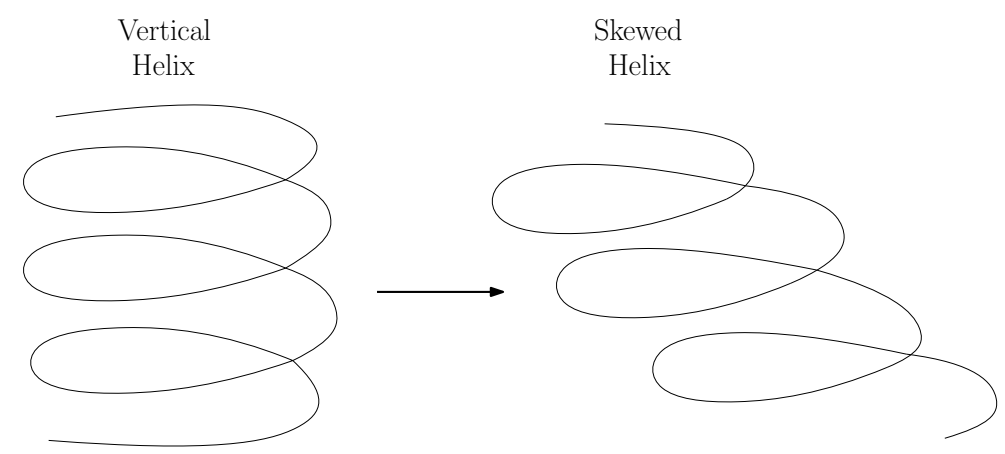

Figure 1.5: Left: Vertical steady spin before the perturbed control application. Right: Slanted helix after the perturbed control application.

control law:

1. Obtain an initial guess for the state variable and control deflection values for an aircraft in a steady, vertical spin for given values of the spinning motion parameters (e.g., the spin rate $\Omega$ ).

2. Using the state values defined in Step 1 as initial values for a numerical root-finding scheme (e.g., Newton-Raphson), with the control deflections held fixed, obtain the true nominal values of the state variables.

3. Linearize the nine equations of motion (three attitude kinematic equations, three translational dynamics equations, and three attitude dynamics equations) about this nominal solution and non-dimensionalize the resulting linear equations.

4. Compute the cycle-averaged inertial velocity of the aircraft when the system is subjected to a pulsewidth modulated (PWM) input in the control deflections, where the period corresponds to the spinning motion and the duty cycle is chosen to effect motion in a desired direction. The resulting state perturbations due to the PWM input are assumed to evolve according to the linear state equations.

5. Compare the cycle-averaged motion with the desired motion to extract the required PWM input.

\subsection{Organization}

The thesis is organized as follows. Chapter 2 provides a brief overview of the problem formulation. Chapter 3 describes the notation and the equations of motion for the aircraft. Chapter 4 describes the problem of finding steady, helical motions. Considering NASA's General Transport Model (GTM) [23], we find and linearize about a steady, helical motion and verify stability by examining the eigenvalues. Chapter 5 
describes the control strategy and chapter 6 presents the results of implementation in flight simulations. We conclude in Chapter 7. 


\section{Chapter 2}

\section{Problem Statement}

As described in chapter 1, the problem comprises two parts.

Problem 1 (Determining a stable, steady, spinning motion). Values for the state and input variables are required which correspond to a stable, steady, vertical spinning motion (in still air). This steady state motion serves as a nominal flight condition from which the control law (Problem 2) induces small perturbations. The nominal control inputs obtained in Problem 1 must therefore not be saturated, so that bi-directional control authority remains available for use in Problem 2.

Problem 2 (Controlling the cycle-averaged velocity direction during descent). A piece-wise constant input is sought to drive the cycle-averaged velocity direction to a desired value. In addition to exhibiting the desired motion, the close-loop system should be robust to model parameter uncertainty and small disturbances. 


\section{Chapter 3}

\section{Simulated Aircraft Model}

The aircraft model used in this chapter is the Matlab representation of the NASA generic transport model (GTM). The GTM is a 5.5\% dynamically scaled, remotely piloted, civil transport aircraft, which was developed for NASA's Aviation Safety Program [29]. Previous studies have shown that the GTM has good spin characteristics [6], which is why this model is chosen for the current study. An analytical nonlinear aerodynamic model for this aircraft (as opposed to a look-up table of aerodynamic data) is available in [23]. This made it possible to code a nonlinear aerodynamic model with lower computer memory requirements, and to formulate smooth analytical functions for control applications.

In this chapter, I first introduce the notation that will be used throughout the thesis. Next I describe the nonlinear six-degree-of-freedom equations of motion for a rigid aircraft in atmospheric flight. The Matlab model for the GTM used in this study is based on these equations.

\subsection{Notation}

The general notation used throughout the thesis largely follows convention (e.g. see [19] for standard flight dynamics notation). Additional notation is introduced as needed. The aircraft attitude relative to inertial space is parameterized by the conventional $X-Y-Z$ Euler angles $\phi, \theta$, and $\psi$. The velocity of the aircraft with respect to inertial space, but expressed in a body-fixed frame, has components $u, v$, and $w$. The body angular velocity vector has components $p, q$, and $r$. We let $F_{x}, F_{y}$, and $F_{z}$ denote the aerodynamic force components in the body frame and we let $\mathscr{L}, \mathscr{M}$, and $\mathscr{N}$ denote the aerodynamic moments. The aircraft's total speed relative to the air is $V=\sqrt{u^{2}+v^{2}+w^{2}}$. The drag force, aerodynamic side force, and lift force acting on the aircraft are denoted $D, Y$, and $L$, respectively, with corresponding non-dimensional force coefficients $C_{D}$, $C_{Y}$, and $C_{L}$. These force coefficients depend on the aerodynamic angles $\alpha$ and $\beta$, the angle of attack and side-slip angle, among other state and control variables. The moment coefficients corresponding to $\mathscr{L}, \mathscr{M}$, 


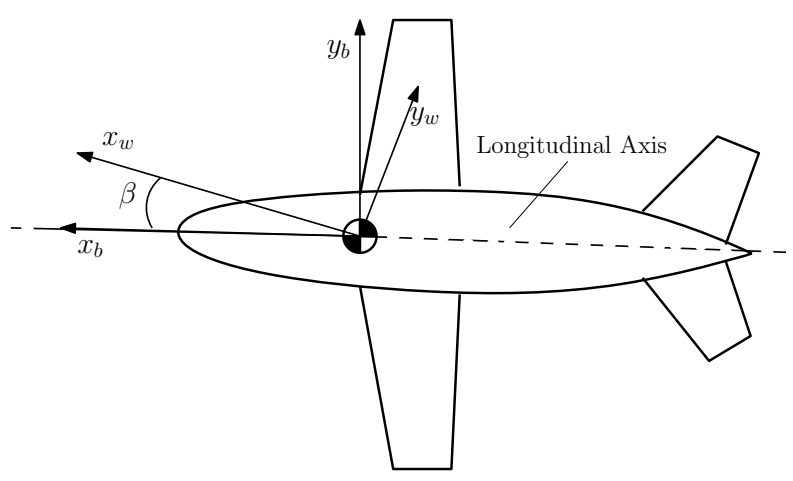

(a) Top View

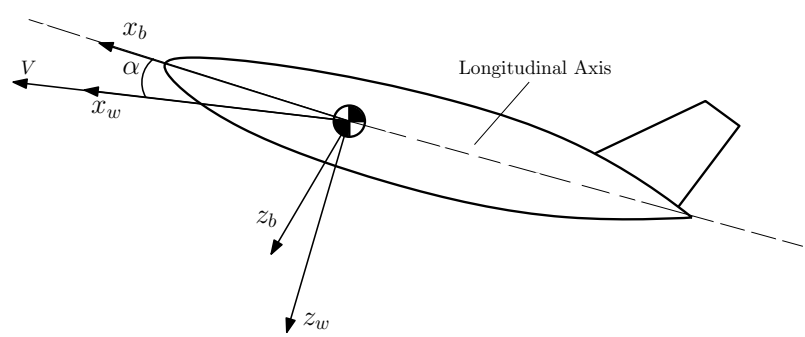

(b) Side View

Figure 3.1: A schematic diagram of the body and wind coordinate frames with respect to the aircraft. Here $\alpha$ is the angle of attack, and $\beta$ is the sideslip angle of the aircraft.

and $\mathscr{N}$ are denoted $C_{l}, C_{m}$, and $C_{n}$, respectively. The aileron, elevator and rudder defections are denoted $\delta_{a}$, $\delta_{e}$, and $\delta_{r}$, respectively.

The aircraft wingspan is denoted $b$ and the mean aerodynamic chord length is $c$. The wing planform area is $S$. Air density is denoted $\rho$. The aircraft mass is denoted $m$ and its weight is $W$. Because of symmetry, the nonzero elements of the inertia matrix include only the diagonal elements $\left\{I_{x}, I_{y}, I_{z}\right\}$ and the cross-product of inertia $I_{x z}$.

Define a body-fixed, orthonormal reference frame centered at the geometric center of the aircraft and represented by the unit vectors $\boldsymbol{b}_{1}, \boldsymbol{b}_{2}$ and $\boldsymbol{b}_{3}$. The vector $\boldsymbol{b}_{1}$ is aligned with the longitudinal axis of the aircraft, $\boldsymbol{b}_{2}$ points out of the right wing, and $\boldsymbol{b}_{3}$ completes the right handed triad; see Fig. 3.1 for a schematic representation of the body frame with respect to the aircraft. Define another orthonormal reference frame, denoted by the unit vectors $\boldsymbol{i}_{1}, \boldsymbol{i}_{2}$ and $\boldsymbol{i}_{3}$, which is fixed in inertial space such that $\boldsymbol{i}_{3}$ is aligned with the force due to gravity. The relative orientation of these two frames is given by the proper rotation matrix $\boldsymbol{R}_{\mathrm{IB}}$, which maps free vectors from the body-fixed reference frame into the inertial reference frame. Let $\boldsymbol{e}_{i}$ represent

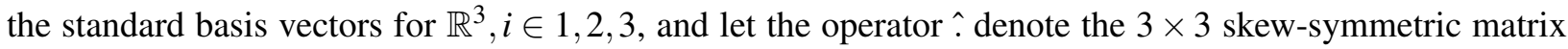
satisfying $\hat{\boldsymbol{a}} \boldsymbol{b}=\boldsymbol{a} \times \boldsymbol{b}$ for vectors $\boldsymbol{a}$ and $\boldsymbol{b}$. Then in terms of conventional Euler angles (roll angle $\phi$, pitch angle $\theta$, and yaw angle $\psi$ ), we have

$$
\boldsymbol{R}_{\mathrm{IB}}(\phi, \theta, \psi)=e^{\widehat{\boldsymbol{e}_{3}} \psi} e^{\widehat{\boldsymbol{e}_{2}} \theta} e^{\widehat{\boldsymbol{e}_{1}} \phi}
$$

The orthonormal wind reference frame, which is commonly used in aircraft dynamics, is defined to be centered at the geometric center of the aircraft and represented by the unit vectors $\boldsymbol{w}_{1}, \boldsymbol{w}_{2}$ and $\boldsymbol{w}_{3}$. The vector $\boldsymbol{w}_{1}$ is aligned with the relative aircraft velocity, and $\boldsymbol{w}_{3}$ lies in the plane of symmetry of the vehicle. The vector $\boldsymbol{w}_{2}$ completes the right handed orthogonal co-ordinate system. The relative orientation of the body frame to the wind frame is given by the proper rotation matrix $\boldsymbol{R}_{\mathrm{BW}}$ : 


$$
\boldsymbol{R}_{\mathrm{BW}}(\alpha, \beta)=e^{-\widehat{\boldsymbol{e}_{2}} \alpha} e^{\widehat{\boldsymbol{e}_{3}} \beta}
$$

where,

$$
\alpha=\tan ^{-1} \frac{u}{w}, \quad \beta=\sin ^{-1} \frac{v}{V}
$$

is the angle of attack and the sideslip angle respectively. Refer to Fig. 3.1 for a schematic diagram of the wind frame with respect to the aircraft.

Similarly, the relative orientation of the wind frame with respect to the inertial frame is given by the proper rotation matrix $\boldsymbol{R}_{\mathrm{IW}}$ :

$$
\boldsymbol{R}_{\mathrm{IW}}(\mu \gamma, \chi)=e^{\widehat{\boldsymbol{e}_{3}} \chi} e^{\widehat{\boldsymbol{e}_{2}} \gamma} e^{\widehat{\hat{e}_{1}} \mu}
$$

Refer to Fig. 3.2 for a schematic diagram summarizing the coordinate frame transformations. From the diagram it can be observed that

$$
\boldsymbol{R}_{\mathrm{IW}}=\boldsymbol{R}_{\mathrm{IB}} \boldsymbol{R}_{\mathrm{BW}}
$$

In the rest of the thesis (3.1) is used to represent $\boldsymbol{R}_{\mathrm{IW}}$ as a function of $(\phi, \theta, \psi, \alpha, \beta)$. We will not use $(\mu, \gamma, \chi)$ in this context henceforth, and these variables might be assigned to represent other quantities as will be specified later.

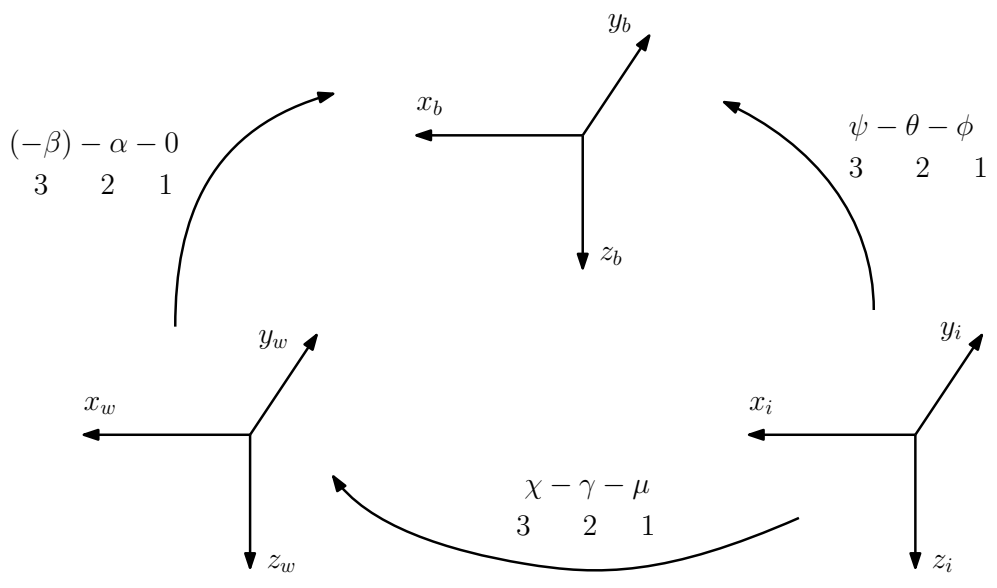

Figure 3.2: A schematic diagram to show the body, wind and inertial coordinate frame transformations. 


\subsection{Equations of Motion}

The location of the body frame with respect to the inertial frame is given by the inertial vector $\boldsymbol{r}=(x, y, z)^{\top}$. Let $\boldsymbol{v}=(u, v, w)^{\top}$ be the translational velocity and let $\boldsymbol{\omega}=(p, q, r)^{\top}$ be the rotational velocity of the aircraft with respect to the inertial space, where both $\boldsymbol{v}$ and $\boldsymbol{\omega}$ are expressed in the body frame. Then the kinematic equations are

$$
\begin{aligned}
\dot{\boldsymbol{r}} & =\boldsymbol{R}_{\mathrm{IB}}(\phi, \theta, \psi) \boldsymbol{v} \\
\dot{\boldsymbol{R}}_{\mathrm{IB}} & =\boldsymbol{R}_{\mathrm{IB}}(\phi, \theta, \psi) \hat{\boldsymbol{\omega}}
\end{aligned}
$$

In terms of the conventional Euler angles notation (3.3) can be replaced by:

$$
\left(\begin{array}{c}
\dot{\phi} \\
\dot{\theta} \\
\dot{\psi}
\end{array}\right)=\left(\begin{array}{ccc}
1 & \sin \phi \tan \theta & \cos \phi \tan \theta \\
0 & \cos \phi & -\sin \phi \\
0 & \sin \phi \sec \theta & \cos \phi \sec \theta
\end{array}\right) \omega
$$

We will denote this in short-hand notation as $\dot{\boldsymbol{\Phi}}=\boldsymbol{L}_{\mathrm{IB}}(\phi, \theta) \boldsymbol{\omega}$, where $\boldsymbol{\Phi}=\left(\begin{array}{ccc}\phi & \theta & \psi\end{array}\right)^{\top}$ and $\boldsymbol{L}_{\mathrm{IB}}(\phi, \theta)=\left(\begin{array}{ccc}1 & \sin \phi \tan \theta & \cos \phi \tan \theta \\ 0 & \cos \phi & -\sin \phi \\ 0 & \sin \phi \sec \theta & \cos \phi \sec \theta\end{array}\right)$.

Let $m$ be the mass of the aircraft. Then the mass matrix $\boldsymbol{M}$ is defined as $\boldsymbol{M}=m \mathbb{I}$. Let $\boldsymbol{I}$ be the moment of inertia matrix of the aircraft in the body frame, given by

$$
\boldsymbol{I}=\left(\begin{array}{ccc}
I_{\mathrm{x}} & I_{\mathrm{xy}} & I_{\mathrm{xz}} \\
I_{\mathrm{xy}} & I_{\mathrm{y}} & I_{\mathrm{yz}} \\
I_{\mathrm{xz}} & I_{\mathrm{yz}} & I_{\mathrm{z}}
\end{array}\right)
$$

Assumption 1. Throughout the rest of the thesis it is assumed that $I_{x y}=I_{y z}=0$. That is the aircraft has a longitudinal symmetry.

Define the linear momentum as $\boldsymbol{p}=\boldsymbol{M} \boldsymbol{v}$ and the angular momentum as $\boldsymbol{h}=\boldsymbol{I} \boldsymbol{\omega}$. The dynamic equations of motion are given by:

$$
\begin{aligned}
\dot{\boldsymbol{p}} & =\boldsymbol{p} \times \boldsymbol{\omega}+m g \boldsymbol{R}_{\mathrm{BI}}(\phi, \theta, \psi) \boldsymbol{e}_{3}+\boldsymbol{F}_{\mathrm{visc}} \\
\dot{\boldsymbol{h}} & =\boldsymbol{h} \times \boldsymbol{\omega}+\boldsymbol{T}_{\mathrm{visc}}
\end{aligned}
$$

Let the thrust force acting on the aircraft be $\boldsymbol{T}$. Then $\boldsymbol{F}_{\text {visc }}$ is the sum of the aerodynamic and thrust forces 
on the aircraft, and is given by:

$$
\boldsymbol{F}_{\text {visc }}=\left(F_{x}+T, F_{y}, F_{z}\right)^{\top}
$$

Assumption 2. Throughout the rest of the thesis it is assumed that the aircraft has no thrust forces acting on it unless specified otherwise. So $T=0$.

$\boldsymbol{T}_{v i s c}$ are the moments acting on the aircraft due to the aerodynamic forces, and are defined as:

$$
\boldsymbol{T}_{\text {visc }}=(\mathscr{L}, \mathscr{M}, \mathscr{N})^{\top}
$$

The aerodynamic forces $F_{x}, F_{y}, F_{z}$ depend on the force coefficients $C_{L}, C_{D}, C_{Y}$, and the aerodynamic moments $\mathscr{L}, \mathscr{M}, \mathscr{N}$ depend on the moment coefficients $C_{l}, C_{m}, C_{n}$. These coefficients in turn are a nonlinear function of the angle of attack $\alpha$, the sideslip angle $\beta$, the body angular rates $p, q, r$, the control inputs $\delta_{a}, \delta_{e}, \delta_{r}$, the mean aircraft chord $c$, the aircraft span $b$ and the air density $\rho$. The exact aerodynamic model used in this thesis is given in appendix A.

Assumption 3. For all the computations, the air density $\rho$ is assumed to be constant and equal to $1.225 \frac{\mathrm{kg}}{\mathrm{m}^{3}}$.

Note that $\dot{\boldsymbol{\Phi}}, \dot{\boldsymbol{p}}$ and $\dot{\boldsymbol{h}}$, do not depend on $\dot{\boldsymbol{r}}$. Therefore, for the rest of the computations, we consider our state vector to be $\zeta=\left(\boldsymbol{\Phi}^{\top}, \boldsymbol{p}^{\top}, \boldsymbol{h}^{\top}\right)^{\top}$. Correspondingly, we will consider the following reduced set of equations:

$$
\begin{aligned}
\dot{\boldsymbol{\Phi}} & =\boldsymbol{L}_{\mathrm{IB}}(\boldsymbol{\phi}, \theta) \boldsymbol{\omega} \\
\dot{\boldsymbol{p}} & =\boldsymbol{p} \times \boldsymbol{\omega}+m g \boldsymbol{R}_{\mathrm{BI}}(\phi, \theta, \psi) \boldsymbol{e}_{3}+\boldsymbol{F}_{\mathrm{visc}} \\
\dot{\boldsymbol{h}} & =\boldsymbol{h} \times \boldsymbol{\omega}+\boldsymbol{T}_{\mathrm{visc}}
\end{aligned}
$$




\section{Chapter 4}

\section{Nominal Solution for Spinning Descent}

The equations of motion and the aerodynamic model implemented in this chapter are nonlinear, and the system exhibits a large family of steady, spinning flight conditions as demonstrated by the bifurcation diagram for the GTM in [41] and later in this chapter in section 4.4. Typically, an aircraft enters spin via a jump condition due to an instability at a co-existing low- $\alpha$ branch of steady states. Thus, in computing a desired numerical trim solution, it is important to provide an accurate initial guess.

One approach to obtain an approximate steady steep spin solution for the six-dimensional system $(u, v, w, p, q, r)$ is described in [47] and more recently in [6]. This method is specialized here for the eight dimensional system $(\phi, \theta, u, v, w, p, q, r)$ by imposing additional constraints. The solution thus obtained is used as the starting point for a continuation method to obtain a manifold of equilibria with the varying control inputs $\delta_{r}, \delta_{e}, \delta_{a}$.

I have implemented a one-parameter continuation method in $\delta_{r}$, varying the rudder input in a specified interval, keeping the other two control inputs fixed at any time. At the end of the cycle, one of the other control inputs $\left(\delta_{a}, \delta_{e}\right)$ is varied by a small angle and the process is repeated. Two and three-parameter continuation methods exist, see [35] for reference. Though they are complicated to implement, they are computationally more efficient than a one-parameter continuation method and provide a more accurate bifurcation diagram.

In the rest of the chapter, first the method used to obtain an initial estimate is described in section 4.1. Next the artificial parameter continuation method based on pseudo-arclength is described in section 4.2. I summarize the process used to obtain the family of exact solutions for a spinning descent in section 4.3 and produce the results for the spinning descent solutions in section 4.4. 


\subsection{Solving for an Initial Estimate}

To obtain an initial estimate of a steady spin state, we consider the 8-dimensional system for the reduced state $-(\phi, \theta, u, v, w, p, q, r)$. The equations of motion are given in (3.6) and are reproduced here in (4.1), except the $\psi$ dynamics is ignored here.

$$
\begin{aligned}
\dot{\phi} & =p+q \sin \phi \tan \theta+r \cos \phi \tan \theta, \\
\dot{\theta} & =q \cos \phi-r \sin \phi, \\
\dot{u} & =r v-q w-g \sin \theta+\frac{F_{x}}{m}, \\
\dot{v} & =p w-r u+g \sin \phi \cos \theta+\frac{F_{y}}{m}, \\
\dot{w} & =q u-p v+g \cos \phi \cos \theta+\frac{F_{z}}{m}, \\
\dot{p} & =-d_{0}\left(p q d_{1}+q r d_{2}+\mathscr{L} I_{\mathrm{z}}+\mathscr{N} I_{\mathrm{xz}}\right), \\
\dot{q} & =\frac{1}{I_{\mathrm{y}}}\left(\mathscr{M}+p r\left(I_{\mathrm{z}}-I_{\mathrm{x}}\right)+I_{\mathrm{xz}}\left(r^{2}-p^{2}\right)\right), \\
\dot{r} & =-\frac{1}{d_{0}}\left(p q d_{3}-q r d_{1}+\mathscr{L} I_{\mathrm{xz}}+\mathscr{N} I_{\mathrm{x}}\right)
\end{aligned}
$$

In these equations

$$
d_{0}=I_{\mathrm{xz}}^{2}-I_{\mathrm{x}} I_{\mathrm{z}}, \quad d_{1}=I_{\mathrm{xz}}\left(I_{\mathrm{x}}-I_{\mathrm{y}}+I_{\mathrm{z}}\right), \quad d_{2}=I_{\mathrm{y}} I_{\mathrm{z}}-I_{\mathrm{xz}}^{2}-I_{\mathrm{z}}^{2}, \quad d_{3}=I_{\mathrm{x}}^{2}+I_{\mathrm{xz}}^{2}-I_{\mathrm{x}} I_{\mathrm{z}}
$$

The unknowns are the eight system states $(\phi, \theta, u, v, w, p, q, r)$ and the three control inputs $\left(\delta_{a}, \delta_{e}, \delta_{r}\right)$. Therefore, three more constraints need to be imposed for the system to be well defined. Here, the values of the angle of attack $\alpha$, the tilt angle $\chi$ (which will be defined shortly) and the angular speed $\Omega$ are chosen.

In the rest of the section, first the required angles are defined and, the body translational and angular velocities are expressed in terms of the given quantities for a steep spin. Next, the assumptions imposed to strike out some of the nonlinear terms are described. Finally the translational dynamics is solved, followed by the rotational dynamics and the rotational kinematics to obtain an approximate steady spin state.

\subsubsection{Kinematic Angle Definitions and Assumptions}

For the helical path shown in Fig. 4.1(a), define the constant helix angle $\gamma=\tan ^{-1}\left(\frac{\Omega R}{U_{0}}\right)$. The spinning airplane experiences sideslip because of the helical motion. The sideslip angle $\beta$ is: 


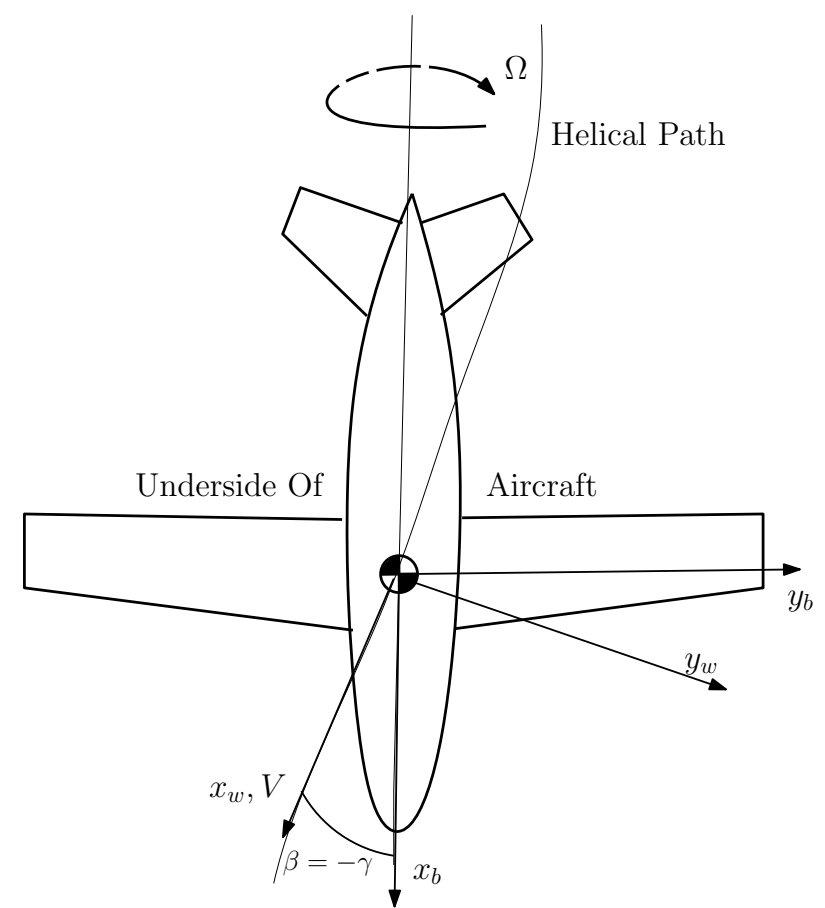

(a) Illustration of the helical path in a steep spin, in an aircraft without wing tilt $(\chi=0)$. The helix angle is defined to be $\gamma=$ $\tan ^{-1} \frac{\Omega R}{U_{0}}$. The sideslip angle in this case is $\beta=-\gamma$.

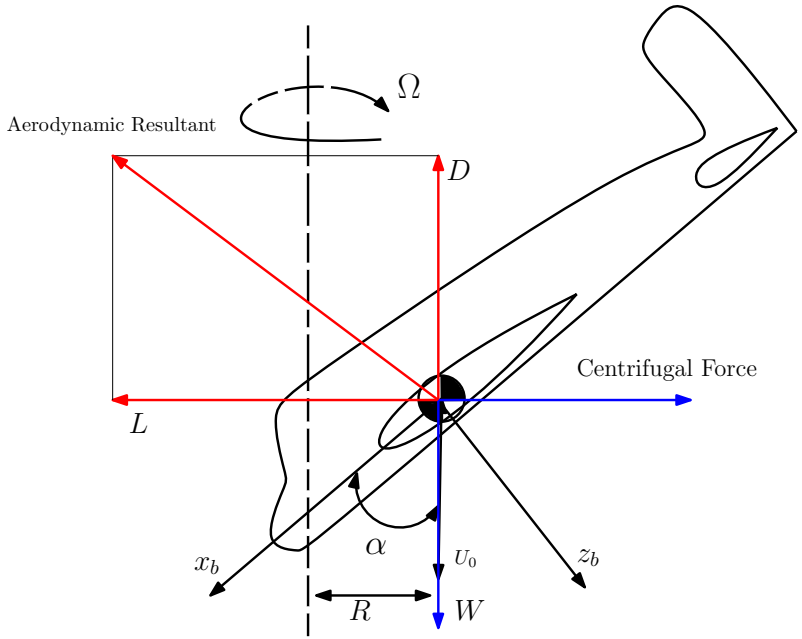

(b) Forces acting on an airplane in a steady steep spin. The aerodynamic forces are in red, and the centrifugal and gravitational force are in blue. The forces in red balance the forces in blue.

Figure 4.1: A schematic of the helical path (left) before the wing tilt and the forces (right) acting on an aircraft after the wing tilt in a steady steep spin. Adapted from [6]

$$
\beta=\sin ^{-1}\left(\frac{v}{V}\right)=\sin ^{-1}\left(\frac{-\Omega R}{V}\right) \sim-\tan ^{-1}\left(\frac{\Omega R}{U_{0}}\right)=-\gamma
$$

Note that the small angle assumption used above will not be valid for high $\frac{\Omega R}{V}$, or when $V \nsim U_{0}$. Here we are solving for a steep spin solution, which implies that $V \sim U_{0}$ and that $R$ is small. In a flight termination scheme, a smaller $R$ implies smaller uncertainty in the aircraft's point of impact, though at the cost of a larger impact speed $V$.

The centrifugal force acting on an aircraft in spin is directed radially outwards and passes through the spin axis, see fig. 4.1(b). So the resultant aerodynamic forces acting on the aircraft need to pass through the spin axis to achieve a moment balance. The sideslip plays an important role in achieving this moment balance. The only way to obtain the required sideslip is via tilting the aircraft wings about the horizontal plane, by rotating the aircraft about the body $\mathrm{z}$ axis. Let $\chi$ denote the angle by which the aircraft is rotated about its body $\mathrm{z}$ axis, see figure 4.2 (a). The resulting wing tilt $\theta_{y}$ with respect to the horizontal plane, as in fig. 4.2(b), is given by:

$$
\sin \left(\theta_{y}\right)=-\cos (\alpha) \sin (\chi)
$$




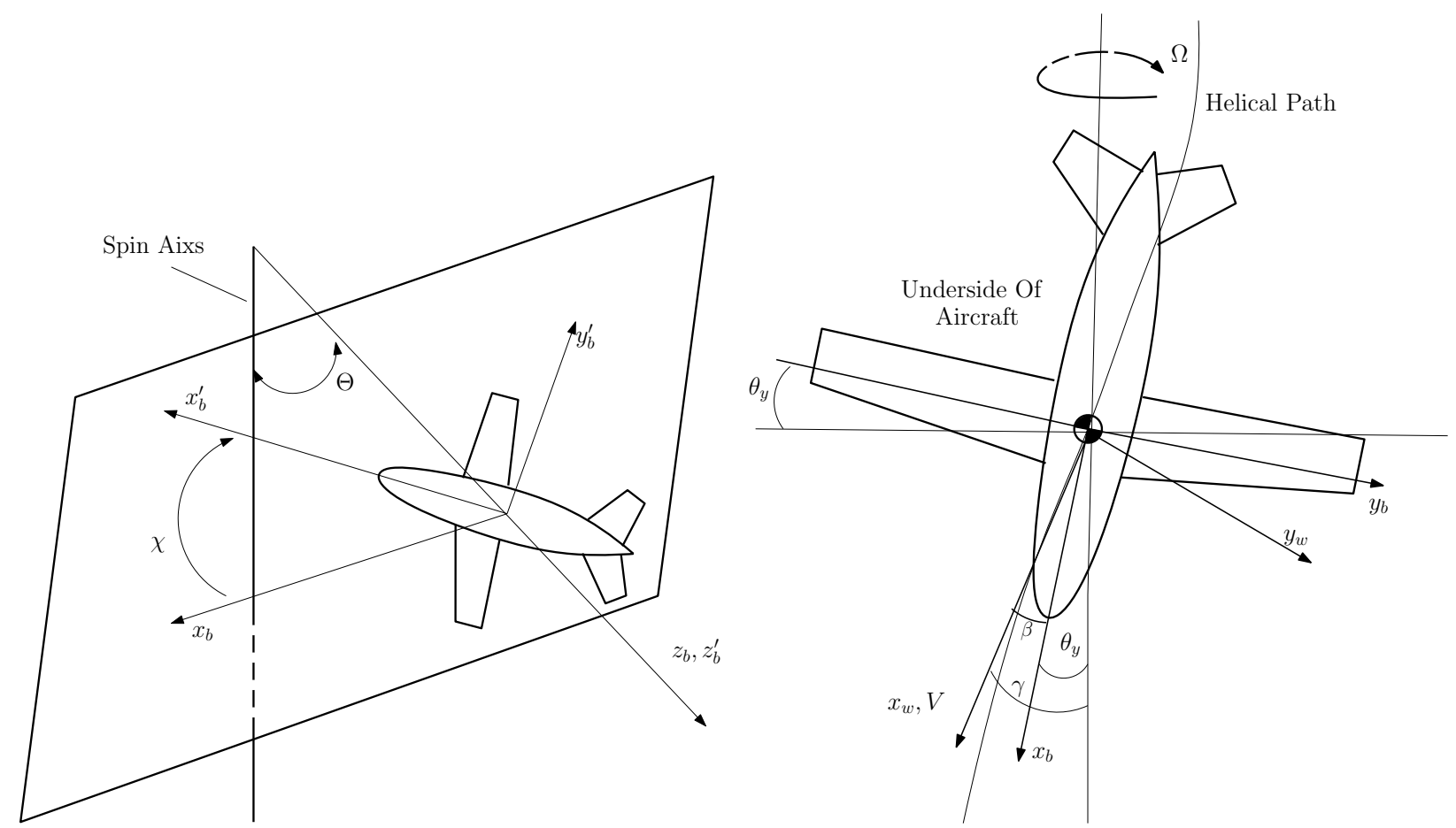

(a) The aircraft is initially oriented along the $x_{b}-y_{b}-z_{b}$ axes. (b) The aircraft rotation about its body z axis by an angle $\chi$ reIt rotates in the plane formed by the $x_{b}-y_{b}$ axes, about the body sults in a wing tilt with respect to the horizontal plane by an angle $\mathrm{z}$ axis by angle $\chi$. It is now oriented along the $x_{b}^{\prime}-y_{b}^{\prime}-z_{b}^{\prime}$ axes. $\theta_{y}$. The sideslip angle after the wing tilt is $\beta=\theta_{y}-\gamma$.

The angle formed by the body $\mathrm{z}$ axis with the spin axis remains constant.

Figure 4.2: A schematic of the kinematic angle $\chi$ (left) the resulting wing tilt $\theta_{y}$ (right) with respect to the horizontal plane. Adapted from [49] and [6].

The side-slip angle after the wing tilt is:

$$
\beta=\theta_{y}-\gamma
$$

The body angular velocity components in this orientation are:

$$
p=\Omega \cos (\alpha) \cos (\chi), \quad q=\Omega \cos (\alpha) \sin (\chi), \quad r=\Omega \sin (\alpha)
$$

It is assumed that $\chi$ doesn't have significant effect on the body velocity vector, which is given by $\boldsymbol{v}_{B}=$ $\boldsymbol{R}_{\mathrm{BW}}\left(V w_{1}\right)$ or explicitly as:

$$
u=V \cos \alpha \cos \beta, \quad v=V \sin \beta, \quad w=V \sin \alpha \cos \beta
$$

Assumption 4. In seeking an initial estimate of the state and control variables for spinning flight, we make the following simplifying assumptions in solving the translational equations to get rid of some of the 
nonlinearities:

(a) The deviation angle $\chi$ of the wings about the aircraft's body $z$ axis is negligible.

(b) The deviation angle $\chi$ has a negligible effect on the body velocity vector, which is given by 4.7.

(c) The side-slip angle is small so that $\cos \beta \sim 1$ and $\sin \beta \sim-\frac{\Omega R}{U_{0}}$.

(d) The speed of descent $U_{0}$ is almost equal to the total speed of the aircraft $V$.

(e) The sideslip forces $F_{y}$ are negligible.

(f) The elevator angle $\delta_{e}$ is negligible.

Note that many of these variables and parameters play an important role in the attitude dynamics, where these assumptions are not valid.

\subsubsection{Translational Dynamics}

In this subsection, assumption 4 is used to strike out some of the nonlinear terms in the translational equations. Then for the given values of $\Omega$ and $\alpha$, the aircraft speed $V$ is solved for a steady steep spin.

For an aircraft in steady spin, the body velocity components are constant, so the rate of change of the linear momentum is zero: $\dot{\boldsymbol{p}}=0$. Let $U_{0}$ be the constant speed of descent and let $R$ denote the radius of the circle that the helical path describes when viewed from above, see fig. 4.1(b). By assumption 4(a), $\chi=0$. So the aircraft wings are assumed to be in the horizontal plane; see fig. 4.1(a).

On applying assumptions 4(a) to the body angular velocity components in (4.6) we get:

$$
p=\Omega \cos \alpha, \quad q=0, \quad r=\Omega \sin \alpha
$$

On applying assumptions 4(b), 4(c) and 4(d) to the body velocity components in (4.7) we get:

$$
u=U_{0} \cos \alpha, \quad v=-\Omega R, \quad w=U_{0} \sin \alpha
$$

By assumptions 4(a), 4(c), and 4(e), the forces acting on the vehicle are given by

$$
F_{x}=L \sin \alpha-D \cos \alpha, \quad F_{y}=0, \quad F_{z}=-L \cos \alpha-D \sin \alpha
$$


The components of the aircraft weight $\boldsymbol{W}$ in the body frame are:

$$
W_{x}=W \cos \alpha, \quad W_{y}=0, \quad W_{z}=W \sin \alpha
$$

The translational equations from (4.1) are:

$$
\begin{aligned}
m \dot{u} & =m(r v-q w)+F_{x}+W_{x} \\
m \dot{v} & =m(p w-r u)+F_{y} \\
m \dot{w} & =m(q u-p v)+F_{z}+W_{z}
\end{aligned}
$$

On substituting equations (4.8)-(4.11) into (4.13) and setting the velocity rates to zero for a steady spin we get:

$$
\begin{aligned}
L \sin \alpha-D \cos \alpha+W \cos \alpha & =m \Omega^{2} R \sin \alpha, \\
-L \cos \alpha-D \sin \alpha+W \sin \alpha & =-m \Omega^{2} R \cos \alpha .
\end{aligned}
$$

Solving (4.13) for the lift and drag forces, we get:

$$
L=m \Omega^{2} R \quad \text { and } \quad D=W
$$

Define dynamic pressure as $Q=\frac{\rho V^{2}}{2}$. Then the lift and drag coefficients are defined in the standard manner as: $C_{L}=\frac{L}{Q S}, C_{D}=\frac{D}{Q S}$. Substituting the values of $L$ and $D$ from (4.14) in their respective force coefficients we get:

$$
C_{L}=\frac{2\left(m \Omega^{2} R\right)}{\rho V^{2} S} \quad \text { and } \quad C_{D}=\frac{2 W}{\rho V^{2} S}
$$

Now solving (4.15) for $V$ and $R$ results in:

$$
V=\sqrt{\frac{2 W}{\rho S C_{D}}} \quad \text { and } \quad R=\frac{\rho V^{2} S C_{L}}{2 m \Omega^{2}}
$$

Here $C_{L}$ and $C_{D}$ are given by the aerodynamic model for a GTM from [23].

$$
\begin{aligned}
& C_{D}=\theta_{1}+\theta_{2} \alpha+\theta_{3} \alpha \bar{q}+\theta_{4} \alpha \delta_{e}+\theta_{5} \alpha^{2}+\theta_{6} \alpha^{2} \bar{q}+\theta_{7} \alpha^{2} \delta_{e}+\theta_{8} \alpha^{3}+\theta_{9} \alpha^{3} \bar{q}+\theta_{10} \alpha^{4}, \\
& C_{L}=\theta_{16}+\theta_{17} \alpha+\theta_{18} \bar{q}+\theta_{19} \delta_{e}+\theta_{20} \alpha \bar{q}+\theta_{21} \alpha^{2}+\theta_{22} \alpha^{3}+\theta_{23} \alpha^{4}
\end{aligned}
$$

$\theta_{i}$ above are the fixed numerical coefficients which depend on the aircraft model considered, and $\bar{q}$ is the non-dimensional pitch rate which is defined as: $\bar{q}=\frac{c q}{2 V}$. By assumption 4 (f), $\delta_{e}=0$ and by (4.8), $\bar{q}=0$. So $C_{D}$ and $C_{L}$ simplify to 


$$
\begin{aligned}
& C_{D}=\theta_{1}+\theta_{2} \alpha+\theta_{5} \alpha^{2}+\theta_{8} \alpha^{3}+\theta_{10} \alpha^{4}, \\
& C_{L}=\theta_{16}+\theta_{17} \alpha+\theta_{21} \alpha^{2}+\theta_{22} \alpha^{3}+\theta_{23} \alpha^{4}
\end{aligned}
$$

and are a function of $\alpha$ alone. Therefore, for any given values of $\Omega$ and $\alpha$, we have found the approximate values of $V$ and $R$ for a steep steady vertical spin.

\subsubsection{Rotational Dynamics}

In this subsection, for given values of $\alpha, \chi$ and $\Omega$, using the approximate aircraft speed $V$ from (4.16) the rotational equations of motion are solved and the required control deflections are computed to maintain a steep steady spin.

For a steady spin, the rate of change of the angular momentum is zero: $\boldsymbol{h}=0$. As mentioned in assumption 4, $\chi$ is assumed to be non-zero for the attitude dynamics equations. The body angular velocity components in this case are given by (4.6).

The rotational equations of motion from (4.1) are rearranged and rewritten here for convenience:

$$
\begin{aligned}
\mathscr{L} & =\dot{p} I_{x}+\dot{r} I_{x z}+I_{x z} p q+\left(I_{z}-I_{y}\right) q r \\
\mathscr{M} & =\dot{q}+I_{x z}\left(r^{2}-p^{2}\right)+\operatorname{pr}\left(I_{x}-I_{z}\right) \\
\mathscr{N} & =\dot{p} I_{x z}+\dot{r} I_{z}+(I y-I x) p q-I_{x z} q r
\end{aligned}
$$

The moments $\mathscr{L}, \mathscr{M}, \mathscr{N}$ are defined in terms of their respective moment coefficients as:

$$
\mathscr{L}=Q S b C_{l}, \quad \mathscr{M}=Q S c C_{m}, \quad \mathscr{N}=Q S b C_{n}
$$

The moment coefficients are in turn defined by the aerodynamic model used, here for a GTM from [23].

$$
\begin{aligned}
C_{l} & =\theta_{24} \beta+\theta_{25} \bar{p}+\theta_{26} \bar{r}+\theta_{27} \delta_{a}+\theta_{28} \delta_{r}, \\
C_{m} & =\theta_{29}+\theta_{30} \alpha+\theta_{31} \bar{q}+\theta_{32} \delta_{e}+\theta_{33} \alpha \bar{q}+\theta_{34} \alpha^{2} \bar{q}+\theta_{35} \alpha^{2} \delta_{e}+\theta_{36} \alpha^{3} \bar{q}+\theta_{37} \alpha^{3} \delta_{e}+\theta_{38} \alpha^{4}, \\
C_{n} & =\theta_{39} \beta+\theta_{40} \bar{p}+\theta_{41} \bar{r}+\theta_{42} \delta_{a}+\theta_{43} \delta_{r}+\theta_{44} \beta^{2}+\theta_{45} \beta^{3} .
\end{aligned}
$$

In the LHS of (4.19), substitute the moments $(\mathscr{L}, \mathscr{M}, \mathscr{N})$ by their definition in terms of the moment coefficients $\left(C_{l}, C_{m}, C_{n}\right)$ from (4.20), and then substitute the moment coefficients by the aerodynamic model in (4.21). In the RHS of (4.19), substitute the angular velocities from (4.6) and set the angular velocity rates to zero for a steady motion. This finally gives us: 


$$
\begin{aligned}
\theta_{24} \beta & +\theta_{25} \bar{p}+\theta_{26} \bar{r}+\theta_{27} \delta_{a}+\theta_{28} \delta_{r} \\
= & \frac{1}{Q S b} \frac{\Omega^{2}}{2}\left(I_{x z} \cos ^{2} \alpha \sin 2 \chi-\left(I_{z}-I_{y}\right) \sin 2 \alpha \sin \chi\right), \\
\theta_{29} & +\theta_{30} \alpha+\theta_{31} \bar{q}+\theta_{32} \delta_{e}+\theta_{33} \alpha \bar{q}+\theta_{34} \alpha^{2} \bar{q}+\theta_{35} \alpha^{2} \delta_{e}+\theta_{36} \alpha^{3} \bar{q}+\theta_{37} \alpha^{3} \delta_{e}+\theta_{38} \alpha^{4} \\
= & \frac{1}{Q S c}\left(2 I_{x z}\left(\cos ^{2} \alpha \cos ^{2} \chi-\sin ^{2} \alpha\right)-\left(I_{z}-I_{x}\right) \sin 2 \alpha \cos \chi\right), \\
\theta_{39} \beta & +\theta_{40} \bar{p}+\theta_{41} \bar{r}+\theta_{42} \delta_{a}+\theta_{43} \delta_{r}+\theta_{44} \beta^{2}+\theta_{45} \beta^{3} \\
= & \frac{1}{Q S b} \frac{\Omega^{2}}{2}\left(\left(I_{x}-I_{y}\right) \cos ^{2} \alpha \sin 2 \chi-I_{x z} \sin 2 \alpha \sin \chi\right) .
\end{aligned}
$$

Before solving for the required control deflections, we first consolidate terms by introducing the following notation:

$$
\begin{array}{lrlrl}
L_{1}=\frac{I_{x z} p q+\left(I_{z}-I_{y}\right) q r}{Q S b}, & M_{1}= & \frac{I_{x z}\left(r^{2}-p^{2}\right)+p r\left(I_{x}-I_{z}\right)}{Q S c}, & & N_{1}=\frac{(I y-I x) p q-I_{x z} q r}{Q S b}, \\
L_{2}=L_{1}-k_{l}, & M_{2}= & M_{1}-k_{m}, & N_{2}= & N_{1}-k_{n}, \\
k_{l}=\theta_{24} \beta+\theta_{25} \bar{p}+\theta_{26} \bar{r}, & k_{m}= & \theta_{29}+\theta_{30} \alpha+\theta_{31} \bar{q}+\theta_{33} \alpha \bar{q} & & k_{n}=\theta_{39} \beta+\theta_{40} \bar{p}+\theta_{41} \bar{r} \\
& +\theta_{34} \alpha^{2} \bar{q}+\theta_{36} \alpha^{3} \bar{q}+\theta_{38} \alpha^{4}, & & +\theta_{44} \beta^{2}+\theta_{45} \beta^{3}
\end{array}
$$

In the definitions of $L_{1}, M_{1}, N_{1}$, I have used $(p, q, r)$ instead of their values in terms of $(\alpha, \chi, \Omega)$ from (4.6) for the compactness of the notation. Now solving (4.22) for the control deflections we get:

$$
\delta_{a}=\frac{N_{2} \theta_{28}-L_{2} \theta_{43}}{\theta_{28} \theta_{42}-\theta_{43} \theta_{27}}, \quad \delta_{e}=\frac{M_{2}}{\theta_{32}+\theta_{35} \alpha^{2}+\theta_{37} \alpha^{3}}, \quad \delta_{r}=\frac{L_{2} \theta_{42}-N_{2} \theta_{27}}{\theta_{28} \theta_{42}-\theta_{43} \theta_{27}}
$$

Therefore, for a given choice of $\alpha, \chi$ and $\Omega$, we have found an estimate of the required control deflections for a steep steady vertical spinning motion.

\subsubsection{Rotational Kinematics}

For a vertical steady spin the euler angle rates $\dot{\phi}$ and $\dot{\theta}$ are equal to zero. Substituting these conditions into the rotational kinematic equations in (3.4), reproduced and rearranged here for convenience:

$$
\left(\begin{array}{c}
p \\
q \\
r
\end{array}\right)=\left(\begin{array}{ccc}
1 & 0 & -\sin \theta \\
0 & \cos \phi & \cos \theta \sin \phi \\
0 & -\sin \phi & \cos \theta \cos \phi
\end{array}\right)\left(\begin{array}{c}
\dot{\phi} \\
\dot{\theta} \\
\dot{\psi}
\end{array}\right)
$$


results in the body angular velocity expressed in terms of the euler angles and the yaw rate:

$$
\begin{aligned}
p & =-\dot{\psi} \sin \theta, \\
q & =\dot{\psi} \cos \theta \sin \phi, \\
r & =\dot{\psi} \cos \theta \cos \phi .
\end{aligned}
$$

Dividing the expression for $q$ in (4.27) by the expression for $r$ in (4.28) and solving for $\phi$ gives:

$$
\phi=\arctan \left(\frac{q}{r}\right)=\arctan \left(\frac{-\sin (\chi) \cos (\alpha)}{\sin (\alpha)}\right)
$$

Here, we have substituted the values of $q$ and $r$ from (4.6).

We know that $p^{2}+q^{2}+r^{2}=\dot{\psi}^{2}$ from (4.26)-(4.28) and that $p^{2}+q^{2}+r^{2}=\Omega^{2}$ from (4.6). This implies $\dot{\psi}= \pm \Omega$. We assume $\dot{\psi}=\Omega$ in the rest of the computations. Substituting the expression for $p$ from (4.6) into (4.26) and solving for $\theta$ gives:

$$
\theta=\arcsin \left(\frac{-p}{\dot{\psi}}\right)=\arcsin \left(\frac{-\Omega \cos (\alpha) \cos (\chi)}{\Omega}\right)=\arcsin (-\cos (\alpha) \cos (\chi))
$$

\subsubsection{Initial Estimate of States for a Steady Steep Spin}

So far, for a given choice of $\alpha, \chi$ and $\Omega$, I have computed the approximate required controls $-\delta_{a}, \delta_{e}, \delta_{r}$. Equations (4.6), (4.7), (4.29), and (4.30) provide an initial guess for the states of an aircraft in a steady vertical spin. These equations have been gathered below for convenience. Here $\beta$ is given by (4.5), $\theta_{y}$ by (4.4) and $V$ by (4.16).

$$
\begin{aligned}
\phi_{0} & =\arctan \left(\frac{-\sin (\chi) \cos (\alpha)}{\sin (\alpha)}\right), & & \theta_{0}=\arcsin (-\cos (\alpha) \cos (\chi)), \\
u_{0} & =V \cos (\alpha) \cos (\beta), & & p_{0}=\Omega \cos (\alpha) \cos (\chi), \\
v_{0} & =V \sin (\beta), & q_{0} & =-\Omega \cos (\alpha) \sin (\chi), \\
w_{0} & =V \sin (\alpha) \cos (\beta), & r_{0} & =\Omega \sin (\alpha)
\end{aligned}
$$

Note that this approach does not give an exact solution; recall that the deviation angle $\chi$ and the elevator deflection $\delta_{e}$ were assumed to be zero for the translational dynamics but not the rotational dynamics. However, the approach does provide an approximation for the steady state values $-\left\{\phi_{0}, \theta_{0}, u 0, v 0, w 0, p 0, q 0, r 0\right\}$ - that can be used to initialize a recursive trim solver. 


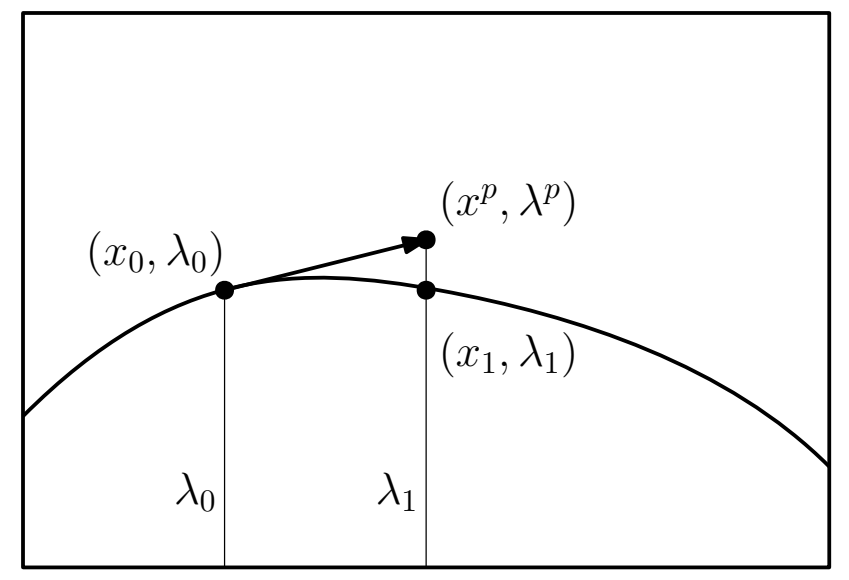

Figure 4.3: A schematic of the predictor-corrector method.

\subsection{Artificial Parameter Continuation Method}

Continuation methods are numerical methods to solve nonlinear equations of the form

$$
\boldsymbol{f}(\boldsymbol{x}, \lambda)=0
$$

Here $f \in \mathbb{R}^{n} \times \mathbb{R} \rightarrow \mathbb{R}^{n}$ is a vector function, $x \in \mathbb{R}^{n}$ in a vector and $\lambda \in I \subset \mathbb{R}$ is a parameter that is varied in the interval $I$. A continuation method doesn't merely solve for a single solution, but generates paths of solutions, i.e. sets of the form:

$$
\Gamma:=\{(\boldsymbol{x}(\lambda), \lambda): \lambda \in I, \boldsymbol{f}(\boldsymbol{x}(\lambda), \lambda)=0\}
$$

Let a dynamical system be defined by the following ODE: $\dot{\boldsymbol{x}}=\boldsymbol{f}(\boldsymbol{x}, \boldsymbol{u})$, where $\boldsymbol{x} \in \mathbb{R}^{n}$ is the state vector, and $\boldsymbol{u} \in \mathbb{R}^{m}$ is the control vector. Therefore, a continuation method can be used to compute the equilibrium solutions of a dynamical system, with the parameters represented by the control vector $u$. At a time, only one control input is varied to obtain the curve of equilibrium points.

The general idea of a continuation method is that of a predictor-corrector scheme. Suppose we have a solution $\left(x_{0}, \lambda_{0}\right)$ on a continuation path. The goal is to find a solution at $\lambda_{0}+\delta \lambda$ for a small perturbation $\delta \lambda$. The first step is to predict the next point on the path for the perturbation $\delta \lambda$. In the next step, the predicted point is corrected towards the solution curve, generally using a Newton's method or some variant of Newton's method, see fig. 4.2. These two steps are iteratively solved by updating the parameter $\lambda$ successively to obtain the entire solution curve. A continuation method performs better compared to other iterative nonlinear solvers due to an adequate prediction of the initial guess for the corrector. Different variants of the continuation method differ in their prediction algorithm. 
For numerically solving a problem, it is important to know the existence of a solution to the problem a priori. So the basic tool for a continuation method is the Implicit Function Theorem (IFT). It ensures the existence of a solution curve $\Gamma$ through a given point $\left(x_{0}, \lambda_{0}\right)$ under certain conditions. IFT can be stated in more general abstract spaces, but for this thesis, a statement based on a Euclidean n-space is sufficient.

Theorem 1. (Implicit Function Theorem) Suppose $\boldsymbol{f}: \mathbb{R}^{n} \times \mathbb{R} \rightarrow \mathbb{R}^{n}, \boldsymbol{x}_{0} \in \mathbb{R}^{n}, \lambda_{0} \in R$ such that

1. $f\left(x_{0}, \lambda_{0}\right)=0$;

2. $\boldsymbol{f}_{\boldsymbol{x}}\left(\boldsymbol{x}_{0}, \lambda_{0}\right)$ is nonsingular;

3. $\boldsymbol{f}, \boldsymbol{f}_{\boldsymbol{x}}\left(\boldsymbol{x}_{0}, \lambda_{0}\right)$ are continuous on the $\operatorname{set}\left\{(\boldsymbol{x}, \lambda):\left\|\boldsymbol{x}-\boldsymbol{x}_{0}\right\|<\varepsilon_{1},\left|\lambda-\lambda_{0}\right|<\varepsilon_{2}\right\}$ for some $\varepsilon_{1}, \varepsilon_{2}>0$.

Then there exists a continuous function $\psi:\left(\lambda_{0}-\varepsilon_{2}, \lambda_{0}+\varepsilon_{2}\right) \rightarrow \mathbb{R}$ such that

- $x_{0}=\psi\left(\lambda_{0}\right)$

- $f(\psi(\lambda), \lambda)=0$ for all $\lambda \in\left(\lambda_{0}-\varepsilon_{2}, \lambda_{0}+\varepsilon_{2}\right)$;

- For each $\lambda \in\left(\lambda_{0}-\varepsilon_{2}, \lambda_{0}+\varepsilon_{2}\right), \psi(\lambda)$ is a unique solution of $\boldsymbol{f}(\boldsymbol{x}, \lambda)=0$ in the set $\left\{\boldsymbol{x}:\left\|\boldsymbol{x}-\boldsymbol{x}_{0}\right\|<\varepsilon_{1}\right\}$.

The proof can be found in any standard book on continuation methods [30]. Therefore, IFT ensures that when a function $\boldsymbol{f}$ satisfies the first three conditions of the hypothesis, (4.32) can be solved locally for $\boldsymbol{x}$ as a function of the parameter $\lambda$. Furthermore, the solution curve thus obtained $\Gamma$, is unique in an appropriate neighborhood. Generally, while implementing a continuation algorithm, condition 2 of the hypothesis is regularly monitored while tracing a continuation curve.

Before giving a detailed formulation of the artificial parameter continuation method, we define a regular solution $\left(\boldsymbol{x}_{0}, \lambda\right)$ of $(4.32)$.

Definition 1. A solution $\left(\boldsymbol{x}_{0}, \lambda_{0}\right)$ of $\boldsymbol{f}(\boldsymbol{x}, \lambda)=0$ is regular if

$$
\operatorname{rank}\left[\boldsymbol{f}_{\boldsymbol{x}}\left(\boldsymbol{x}_{0}, \lambda_{0}\right) \quad \boldsymbol{f}_{\lambda}\left(\boldsymbol{x}_{0}, \lambda_{0}\right)\right]=n
$$

A path $\Gamma:=\{(\boldsymbol{x}(s), \lambda(s)): \boldsymbol{f}(\boldsymbol{x}(s), \lambda(s))=0, s \in I\}$ is regular if all points on $\Gamma$ are regular.

The Implicit Function Theorem guarantees that a unique solution branch passes through any regular point, i.e., any point that satisfies the full-rank condition (4.34). If $\boldsymbol{f}_{\boldsymbol{x}}\left(\boldsymbol{x}_{0}, \lambda_{0}\right)$ is nonsingular at a solution $\left(\boldsymbol{x}_{0}, \lambda_{0}\right)$, then $f_{\boldsymbol{x}}\left(\boldsymbol{x}_{0}, \lambda_{0}\right)$ has rank $n$, and therefore the full rank condition holds. However, if at a regular solution $\boldsymbol{f}_{\boldsymbol{x}}\left(\boldsymbol{x}_{0}, \lambda_{0}\right)$ is singular and has rank $n-1$, then equivalently the null space of $\boldsymbol{f}_{\boldsymbol{x}}\left(\boldsymbol{x}_{0}, \lambda_{0}\right)$ has dimension 1. This happens at a fold. Now for the rank condition in (4.34) to hold, $\boldsymbol{f}_{\lambda}\left(\boldsymbol{x}_{0}, \lambda_{0}\right)$ must not lie in the range space of $f_{\boldsymbol{x}}\left(\boldsymbol{x}_{0}, \lambda_{0}\right)$. This has been summarized in the following theorem. 
Theorem 2. Suppose $\left(\boldsymbol{x}_{0}, \lambda_{0}\right)$ is a solution of $\boldsymbol{f}(\boldsymbol{x}, \lambda)=0$. Then $\left(\boldsymbol{x}_{0}, \lambda_{0}\right)$ is regular if and only if

1. $\boldsymbol{f}_{\boldsymbol{x}}\left(\boldsymbol{x}_{0}, \lambda_{0}\right)$ is nonsingular; $O R$

2. $\operatorname{dimN}\left(\boldsymbol{f}_{\boldsymbol{x}}\left(\boldsymbol{x}_{0}, \lambda_{0}\right)\right)=1$ AND $\boldsymbol{f}_{\lambda}\left(\boldsymbol{x}_{0}, \lambda_{0}\right) \notin$ Range $\boldsymbol{f}_{\boldsymbol{x}}\left(\boldsymbol{x}_{0}, \lambda_{0}\right)$.

Now we define the formulation of an artificial parameter continuation method. It involves defining an artificial parameter $s$ by a constraint equation $g(\boldsymbol{x}, \lambda, s)=0$ and then solving the augmented system

$$
\boldsymbol{F}(\boldsymbol{v}, s):=\left\{\begin{array}{l}
\boldsymbol{f}(\boldsymbol{x}, \lambda)=0 \\
g(\boldsymbol{x}, \lambda, s)=0
\end{array}\right.
$$

The augmented state variable is $\boldsymbol{v}=\left(\begin{array}{ll}\boldsymbol{x} & \lambda\end{array}\right)^{\top}$, and the new system parameter is $s$. Then given a point $\left(\boldsymbol{v}_{0}, s_{0}\right)$ that satisfies $\boldsymbol{F}\left(\boldsymbol{v}_{0}, s_{0}\right)=0$, the next point on the solution curve is predicted in the following three steps:

- Step 1: Increment the artificial parameter $s$ by a step length $\Delta s$.

- Step 2: Define the direction vector along the tangent $\dot{\boldsymbol{v}}_{0}$ to the solution curve at $\left(\boldsymbol{v}_{0}, s_{0}\right)$ according to

$$
\boldsymbol{F}_{\boldsymbol{v}}\left(\boldsymbol{v}_{0}, s_{0}\right) \dot{\boldsymbol{v}}_{0}=-\boldsymbol{F}_{s}\left(\boldsymbol{v}_{0}, s_{0}\right)
$$

Equation 4.36 is obtained by differentiating 4.35 with respect to $s$. So $\dot{v}=\frac{d v}{d s}$.

- Step 3: Predict the updated augmented state variable $v$ by linear extrapolation along the direction vector:

$$
\boldsymbol{v}_{p}:=\boldsymbol{v}_{0}+\Delta s \dot{\boldsymbol{v}}_{0}
$$

Therefore, the next point predicted on the solution curve $\left(\boldsymbol{x}_{p}, \lambda_{p}\right)$ is explicitly given by:

$$
\begin{aligned}
s_{p} & =s_{0}+\Delta s \\
\left(\begin{array}{c}
\boldsymbol{x}_{p} \\
\lambda_{p}
\end{array}\right) & =\left(\begin{array}{c}
\boldsymbol{x}_{0} \\
\lambda_{0}
\end{array}\right)-\Delta s\left(\begin{array}{cc}
\boldsymbol{f}_{\boldsymbol{x}}\left(\boldsymbol{x}_{0}, \lambda_{0}\right) & \boldsymbol{f}_{\lambda}\left(\boldsymbol{x}_{0}, \lambda_{0}\right) \\
g_{\boldsymbol{x}}\left(\boldsymbol{x}_{0}, \lambda_{0}, s_{0}\right)^{\top} & g_{\lambda}\left(\boldsymbol{x}_{0}, \lambda_{0}, s_{0}\right)
\end{array}\right)^{-1}\left(\begin{array}{c}
0 \\
g_{s}\left(\boldsymbol{x}_{0}, \lambda_{0}, s_{0}\right)
\end{array}\right)
\end{aligned}
$$

Next we apply a Newton corrector. It is given in terms of $\boldsymbol{v}$ as

$$
\boldsymbol{v}_{p}^{k+1}=\boldsymbol{v}_{p}^{k}-\left(\boldsymbol{F}_{\boldsymbol{v}}\left(\boldsymbol{v}_{p}^{k}, s_{p}\right)\right)^{-1} \boldsymbol{F}\left(\boldsymbol{v}_{p}^{k}, s_{p}\right)
$$


or equivalently in terms of $x$ and $\lambda$ as

$$
\left(\begin{array}{c}
\boldsymbol{x}_{p}^{k+1} \\
\lambda_{p}^{k+1}
\end{array}\right)=\left(\begin{array}{c}
\boldsymbol{x}_{p}^{k} \\
\lambda_{p}^{k}
\end{array}\right)-\left(\begin{array}{cc}
\boldsymbol{f}_{\boldsymbol{x}}\left(\boldsymbol{x}_{p}^{k}, \lambda_{p}^{k}\right) & \boldsymbol{f}_{\lambda}\left(\boldsymbol{x}_{p}^{k}, \lambda_{p}^{k}\right) \\
g_{\boldsymbol{x}}\left(\boldsymbol{x}_{p}^{k}, \lambda_{p}^{k}, s_{p}\right)^{\top} & g_{\lambda}\left(\boldsymbol{x}_{p}^{k}, \lambda_{p}^{k}, s_{p}\right)
\end{array}\right)^{-1}\left(\begin{array}{c}
\boldsymbol{f}\left(\boldsymbol{x}_{p}^{k}, \lambda_{p}^{k}\right) \\
g_{s}\left(\boldsymbol{x}_{p}^{k}, \lambda_{p}^{k}, s_{p}\right)
\end{array}\right)
$$

Recall that the IFT guarantees the existence of a unique solution curve through any point at which the full rank condition (4.34) holds. A fold is a regular point where the Jacobian of $\boldsymbol{f}$ is singular. A natural parameter method has a $f_{x}^{-1}$ term in the predictor and corrector steps, which is not well-defined at folds. An artificial parameter method circumvents this problem by defining an artificial parameter via $g(\boldsymbol{x}, \lambda, s)=0$ such that, the Jacobian of the augmented system is always nonsingular. The Jacobian of $\boldsymbol{F}$ in the augmented system, takes the form of matrix $\boldsymbol{M}$ in the following theorem, which states conditions on the singularity (or nonsingularity) of $\boldsymbol{M}$.

Theorem 3. Suppose $\boldsymbol{A}, \boldsymbol{b}$, c and d are linear operators, $\boldsymbol{A}: \mathbb{R}^{n} \rightarrow \mathbb{R}^{n}, b: \mathbb{R} \rightarrow \mathbb{R}^{n}, \boldsymbol{c}^{\top}: \mathbb{R}^{n} \rightarrow \mathbb{R}$ and $d: \mathbb{R} \rightarrow \mathbb{R}$ and define

$$
\boldsymbol{M}:=\left(\begin{array}{cc}
\boldsymbol{A} & b \\
\boldsymbol{c}^{\top} & d
\end{array}\right)
$$

1. If $\boldsymbol{A}$ is nonsingular, then $M$ is nonsingular iff $d-\boldsymbol{c}^{\top} \boldsymbol{A}^{-1} \boldsymbol{b} \neq 0$.

2. If $\boldsymbol{A}$ is singular with $\operatorname{dim} N(\boldsymbol{A})=1$, then $\boldsymbol{M}$ is nonsingular iff $\boldsymbol{b} \notin \operatorname{Range}(\boldsymbol{A})$ and $\boldsymbol{c}^{\top} \notin \operatorname{Range}(A)$.

3. If $\operatorname{dim} N(\boldsymbol{A}) \geq 2$, then $\boldsymbol{M}$ is singular.

The proof can be found in [30].

A pseudoarclength parameterization ensures that Jacobian of $\boldsymbol{F}$ is always nonsingular. It is given by:

$$
g(\boldsymbol{x}, \lambda, s)=\dot{\boldsymbol{x}}_{0}^{\top}\left(\boldsymbol{x}-\boldsymbol{x}_{0}\right)+\dot{\lambda}_{0}\left(\boldsymbol{\lambda}-\lambda_{0}\right)-s
$$

Here the directions $\dot{\boldsymbol{x}}_{0}$ and $\dot{\lambda}_{0}$ are defined according to the normalized direction vector $\dot{\boldsymbol{v}}_{0}$ in (4.36), that is $\hat{\boldsymbol{v}}_{0}=\frac{\dot{\boldsymbol{v}}_{0}}{\left\|\dot{\boldsymbol{v}}_{0}\right\|}$. So at each step $\dot{\boldsymbol{x}}_{0}$ and $\dot{\lambda}_{0}$ are rescaled such that $\left\|\dot{\boldsymbol{x}}_{0}^{\top}\right\|^{2}+\left|\dot{\lambda}_{0}\right|^{2}=1$. The following theorem summarizes the formal result.

Theorem 4. Suppose $\left(\boldsymbol{x}_{0}, \lambda_{0}\right)$ is a regular point for $\boldsymbol{f}(\boldsymbol{x}, \lambda)=0$. Then $\boldsymbol{F}_{\boldsymbol{v}}$ is nonsingular for $g$ defined as in (4.41).

The proof is provided in [31] and is based on theorems 2 and 3. 


\subsection{Exact Nominal Solution}

To solve for the equilibrium curves of (4.1) with the varying control inputs $\left(\delta_{a}, \delta_{e}, \delta_{r}\right)$, start with the controls obtained from (4.24) and feed the initial guess for the state variables from (4.31) into an artificial parameter continuation method using MATLAB. Next, check the stability of the equilibrium solutions by checking the eigenvalues of the Jacobian matrix.

Once a set of equilibrium solutions are obtained, the nominal spin solutions is chosen such that it is stable, and the nominal controls are such that there is still a bidirectional control available to apply perturbed control inputs to slant the helix. Denote the nominal states obtained with a subscript "n": $\left\{\phi_{\mathrm{n}}, \theta_{\mathrm{n}}, u_{\mathrm{n}}, v_{\mathrm{n}}, w_{\mathrm{n}}, p_{\mathrm{n}}, q_{\mathrm{n}}, r_{\mathrm{n}}\right\}$.

\subsection{Results}

Figure 4.4 shows the variation in the equilibrium spin states with a varying rudder input $\delta_{r}$. The aileron, elevator and rudder inputs are bounded such that:

$$
\begin{aligned}
& -15^{\circ}<\delta_{a}<0^{\circ} \\
& 10^{\circ}<\delta_{e}<30^{\circ} \\
& -30^{\circ}<\delta_{r}<30^{\circ}
\end{aligned}
$$

These bounds were chosen to get an almost linear equilibrium manifold. The yellow circles in the figure are the stable equilibria and the blue circles are the unstable equilibria. The black cross is the nominal solution chosen for the control application in chapter 6.

From the plots, it can be observed that around the chosen nominal solution, $\phi, \theta, v, w, q, r$ increase with and increase in $\delta_{r}$, whereas $u, p$ decrease with an increase in $\delta_{r}$. Detailed plots for the equilibria for a wider range of control inputs can be found in appendices B and $\mathrm{C}$. 

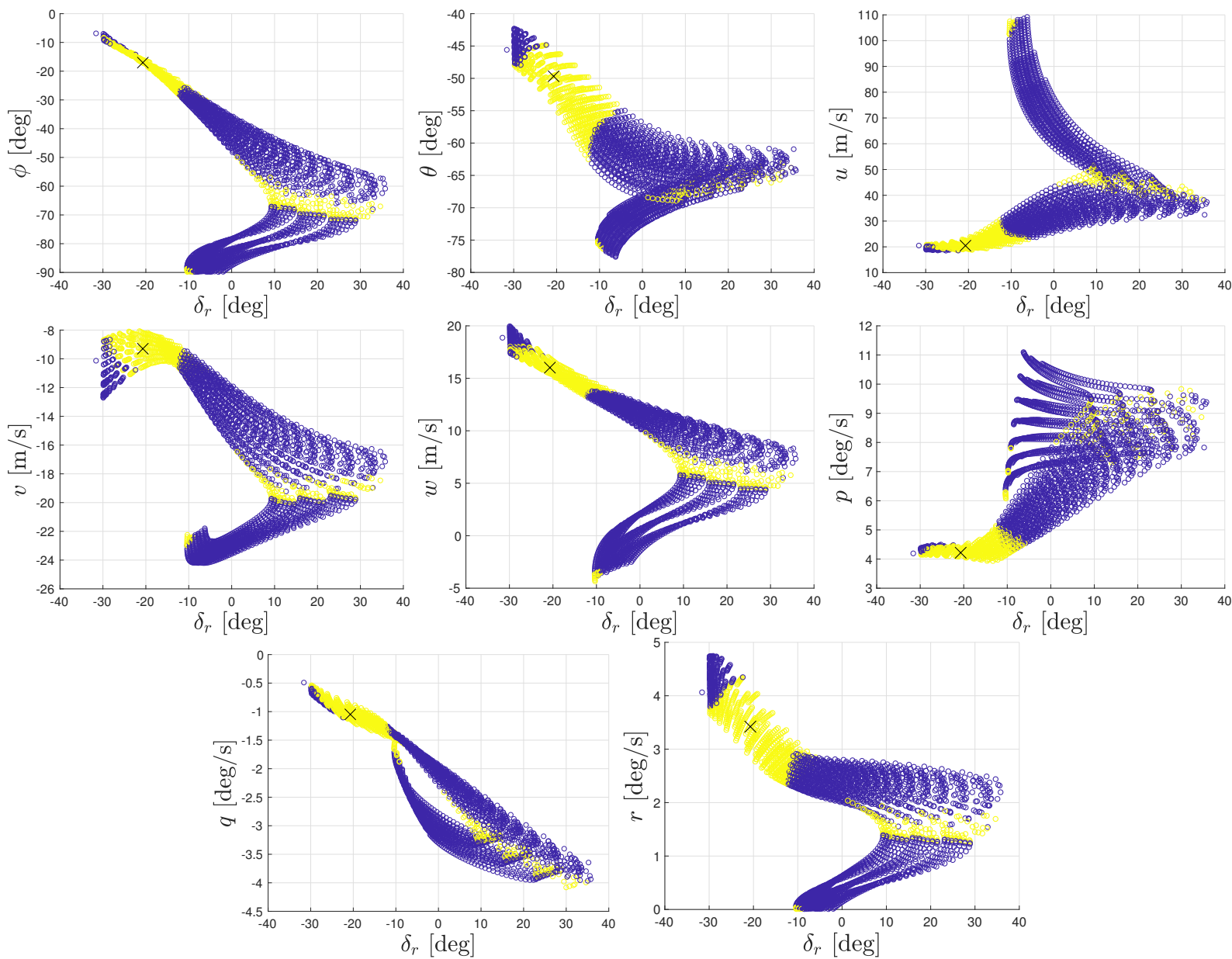

Figure 4.4: Equilibrium spin solutions for $-15<\delta_{a}<0,10<\delta_{e}<30,-30<\delta_{r}<30$. Yellow circles are stable equilibria and blue circles are unstable equilibria. The black cross is the nominal solution chosen for the control application in chapter 6. 


\section{Chapter 5}

\section{Discrete-Time Control Formulation}

Consider the following deterministic continuous-time linear time-varying system $S 1$,

$$
S 1=\left\{\begin{array}{l}
\dot{\boldsymbol{x}}(t)=\boldsymbol{A} \boldsymbol{x}(t)+\boldsymbol{B} \boldsymbol{u}(t), \boldsymbol{x}\left(t_{0}\right)=\boldsymbol{x}_{0} \\
\boldsymbol{y}(t)=\boldsymbol{C}(t) \boldsymbol{x}(t) \\
z(t)=\int_{t_{0}}^{t} \boldsymbol{y}(\tau) d \tau
\end{array}\right.
$$

where $\boldsymbol{x} \in \mathbb{R}^{n}$ is the state vector, $\boldsymbol{u} \in \mathbb{R}^{m}$ in the input vector, $\boldsymbol{y} \in \mathbb{R}^{p}$ is the output vector and $\boldsymbol{z} \in \mathbb{R}^{p}$ is the integrated output vector. Matrices $\boldsymbol{A}$ and $\boldsymbol{B}$ are time-invariant. Matrix $\boldsymbol{C}(t)$ is periodic with a time period $T$. The triplet $\Sigma:=(\boldsymbol{A}, \boldsymbol{B}, \boldsymbol{C}(t))$ is assumed to be known a priori. Both the output measurement $\boldsymbol{y}(t)$ and system states $\boldsymbol{x}(t)$ are assumed to be available. In this chapter we consider the following specific problem.

Problem 3. The objective of this problem is to estimate the constant input signal $\boldsymbol{u}_{0}$, applied over a fraction of the time period $T$, such that the integral of the output signal, defined as $z(t)$, at integer multiples of the time period $T$ is equal to the desired values $z_{d}(k T)$, where $k \in \mathbb{Z}$.

The main assumption imposed to solve this problem is:

Assumption 5. The system $S 1$ is assumed to be square $(m=p)$.

\section{Control Formulation}

Let the fraction of the time period $T$ over which a constant control input is applied be $\Delta T$. Without loss of generality, we develop the control input for one cycle. So the initial time $t_{0}=0$ and the final time $t_{f}=T$. 
The same approach can be iteratively applied for each successive cycle.

$$
\boldsymbol{u}(t)= \begin{cases}\boldsymbol{u}_{0}, & \text { for } 0 \leq t<\Delta T \\ 0, & \text { for } \Delta T \leq t<T\end{cases}
$$

By the variation of parameters formula, $\boldsymbol{y}(t)$ is given by $\boldsymbol{y}(t)=C(t) e^{A t} \boldsymbol{x}_{0}+\int_{t_{0}}^{t} C(t) e^{A(\sigma)} B u(t-\sigma) d \sigma, t \geq 0$. Let $t_{0}=0$ and $\Delta T<t \leq T$. Substituting the control input from (5.2), we get

$$
\boldsymbol{y}(t)=C(t) e^{A t} \boldsymbol{x}_{0}+\int_{0}^{\Delta T} C(t) e^{A(\sigma)} B u_{0} d \sigma
$$

Correspondingly, $z(T)$ is given by:

$$
\boldsymbol{z}(T)=\int_{0}^{T}\left(C(t) e^{A t} \boldsymbol{x}_{0}+\int_{0}^{\Delta T} C(t) e^{A(\sigma)} B u_{0} d \sigma\right) d t
$$

Define $\bar{C}=\int_{0}^{T} C(t) e^{A t} d t$ and $\bar{D}=\int_{0}^{T} C(t)\left(\int_{0}^{\Delta T} e^{A(\sigma)} d \sigma\right) d t B$. Then

$$
z(T)=\bar{C} x_{0}+\bar{D} u_{0}
$$

We want $z(T)=z_{d}(T)$. Equating $z(T)$ from (5.5) to $z_{d}(T)$ we get

$$
u_{0}=\bar{D}^{-1}\left(z_{d}(T)-\bar{C} x_{0}\right)
$$

Remark 1. The advantage of this method is that we need to compute $\bar{C}$ and $\bar{D}$ only once per cycle. The disadvantage is that this method does not account for any disturbances in the state of input variables in the middle of a cycle. This problem can be circumvented by a continuous-time control strategy.

\section{Lower Bound on the Maximum Control Input $\left\|u_{\max }\right\|$ Feasible}

Remark 2. Assuming the linear system assumption is valid, the following section provides a lower bound on the maximum control input feasible to achieve the desired output. However, the bounds are too conservative and are not helpful in a practical application.

Let $\boldsymbol{u}_{\max }$ be the maximum control input allowed, so $\left\|u_{0}\right\| \leq\left\|u_{\max }\right\|$. Then for the control formulation in (5.6), take norms on both the sides to get: 


$$
\left\|\bar{D} \boldsymbol{u}_{0}\right\|=\left\|\boldsymbol{z}_{d}(T)-\bar{C} \boldsymbol{x}_{0}\right\| \leq\|\bar{D}\|\left\|\boldsymbol{u}_{\max }\right\|
$$

Given an initial perturbation in the state $\boldsymbol{x}_{0}$ and a given $\Delta T$, using the last inequality above I find a lower bound on the minimum feasible $\left\|\boldsymbol{u}_{\max }\right\|$ so that the desired output $z_{d}(T)$ can be achieved, for a linear stable system.

In (5.6), the only term that depends on $\Delta T$ is $\bar{D}$, which is defined as $\bar{D}=\int_{0}^{T} C(t)\left(\int_{0}^{\Delta T} e^{A(\sigma)} d \sigma\right) d t B$. Note that $\left(\int_{0}^{\Delta T} e^{A(\sigma)} d \sigma\right)$ does not depend on $t$ and on integrating is equal to $A^{-1}\left(e^{A \Delta T}-\mathbb{I}\right)$. The simplified expression for $\bar{D}$ is then:

$$
\bar{D}=\int_{0}^{T} C(t) A^{-1}\left(e^{A \Delta T}-\mathbb{I}\right) d t B
$$

Let $A=P^{-1} J P$, where $J$ is the Jordan form of $A$ and $P$ is the corresponding matrix of eigenvectors. On substituting $P^{-1} J P$ for $A$ and simplifying $\bar{D}$ we get:

$$
\begin{aligned}
\bar{D} & =\int_{0}^{T} C(t)\left(P^{-1} J P\right)^{-1} P^{-1}\left(e^{J \Delta T}-\mathbb{I}\right) P d t B \\
& =\int_{0}^{T} C(t) P^{-1} J^{-1}\left(e^{J \Delta T}-\mathbb{I}\right) P d t B \\
& =\int_{0}^{T} C(t) d t P^{-1} J^{-1}\left(e^{J \Delta T}-\mathbb{I}\right) P B
\end{aligned}
$$

Define the integral of $C(t)$ over a time period as $\tilde{C}=\int_{0}^{T} C(t) d t$. Then

$$
\bar{D}=\tilde{C} P^{-1} J^{-1}\left(e^{J \Delta T}-\mathbb{I}\right) P B
$$

Now, $\left\|\bar{D} u_{0}\right\| \leq\|\bar{D}\|\left\|u_{0}\right\|$ by the Cauchy-Schwarz inequality. From (5.8) $\|\bar{D}\| \leq\|\tilde{C}\|\left\|P^{-1}\right\|\left\|J^{-1}\right\|\left\|\left(e^{J \Delta T}-\mathbb{I}\right)\right\|\|P\|\|B\|$ by a successive application of the Cauchy-Schwarz inequality. Let $K=\|\tilde{C}\|\left\|P^{-1}\right\|\left\|J^{-1}\right\|\|\| P\|\| B \|$. So we have:

$$
\begin{aligned}
\left\|\bar{D} u_{0}\right\| & \leq\|\bar{D}\|\left\|u_{0}\right\| \\
& \leq\|\bar{D}\|\left\|u_{\max }\right\| \\
& \leq\|\tilde{C}\|\left\|P^{-1}\right\|\left\|J^{-1}\right\|\left\|\left(e^{J \Delta T}-\mathbb{I}\right)\right\|\|P\|\|B\|\|\| u_{\max } \| \\
& \leq K\left\|\left(e^{J \Delta T}-\mathbb{I}\right)\right\|\left\|u_{\max }\right\| \\
& \leq K\left(e^{\sigma_{\max }(J) \Delta T}+1\right)\left\|u_{\max }\right\|
\end{aligned}
$$

In the last step, by the triangle inequality we have $\left\|\left(e^{J \Delta T}-\mathbb{I}\right)\right\| \leq\left\|e^{J \Delta T}\right\|+\|\mathbb{I}\|=\left\|e^{J \Delta T}\right\|+1$. Now, for an 
exponential matrix $\left\|e^{J \Delta T}\right\| \leq e^{\sigma_{\max }(J) \Delta T}$. This gives us $\left\|\left(e^{J \Delta T}-\mathbb{I}\right)\right\| \leq\left(e^{\sigma_{\max }(J) \Delta T}+1\right)$.

Taking a norm on both sides of (5.6) we get $\left\|\bar{D} u_{0}\right\|=\left\|z_{d}(T)-\bar{C} \boldsymbol{x}_{0}\right\|$. So we have:

$$
\left\|\boldsymbol{z}_{d}(T)-\bar{C} \boldsymbol{x}_{0}\right\| \leq K\left(e^{\sigma_{\max }(J) \Delta T}+1\right)\left\|u_{\max }\right\|
$$

For a system with a zero eigenvalue but stable in the rest of the eigen directions we have $\sigma_{\max }(J)=0$ (the system of interest here has all negative eigenvalues except one zero eigenvalue as will be observed in the next chapter). Therefore,

$$
\left\|z_{d}(T)-\bar{C} x_{0}\right\| \leq 2 K\left\|u_{\max }\right\|
$$

or

$$
\left\|u_{\max }\right\| \geq \frac{\left\|z_{d}(T)-\bar{C} x_{0}\right\|}{2 K}
$$

Similar conditions can be obtained for a stable system with no zero eigenvalues and an unstable system.

Remark 3. If the system given by 5.1 is obtained by linearizing a nonlinear system about some nominal condition, then even if the minimum bound on $\left\|\boldsymbol{u}_{\text {max }}\right\|$ is satisfied, the desired integrated output $\boldsymbol{z}_{d}(T)$ might not be atainable if the actual nonlinear system does not satisfy the linear assumption for the corresponding input $\boldsymbol{u}_{0}$ obtained from 5.6. In such cases, there will be further constraints on $\Delta T$ and $\left\|\boldsymbol{u}_{\text {max }}\right\|$ imposed by the validity of the linear assumption, depending on the structure of the nonlinear system. 


\section{Chapter 6}

\section{Application in Simulated Flight Termination}

In this chapter, the method developed in chapter 5 is used to control the direction of the axis of the helical descent of an aircraft in steady vertical spin. The first step is to linearize the nonlinear aircraft dynamical equations to obtain a system of the form (5.1). To this end, first the system is non-dimensionalized. Then it is linearized about a reference nominal equilibrium spin. Next the region of attraction around this equilibrium is studied using Monte-Carlo simulations to get an estimate of the maximum state perturbations allowed. Then the control input for the system is formulated based on the discussion in chapter 5. Finally the control input is applied to slant the helical descent for a range of different angles.

\subsection{Nondimensionalization}

Let the reference quantities for nondimensionalizing distance, time and mass be denoted $S_{0}, T_{0}$ and $m_{0}$, respectively.

$$
S_{0}=b, \quad T_{0}=\frac{2 \pi}{\dot{\psi}_{\mathrm{n}}}, \quad m_{0}=m
$$

where $\dot{\psi}_{\mathrm{n}}$ is given by the corresponding Euler angular rate equation in 6.1.

$$
\dot{\psi}_{\mathrm{n}}=q_{\mathrm{n}} \sin \phi_{\mathrm{n}} \sec \theta_{\mathrm{n}}+r_{\mathrm{n}} \cos \phi_{\mathrm{n}} \sec \theta_{\mathrm{n}}
$$

Accordingly, the nondimensional distance, time and mass are given by:

$$
\hat{s}=\frac{s}{S_{0}}, \quad \tau=\frac{t}{T_{0}}, \quad \hat{m}=\frac{m}{m_{0}}
$$


The nondimensional velocity, angular velocity, force, moment and moment of inertia about the body $x$-axis are defined below. We have also defined the nondimensional acceleration due to gravity and the derivative of a quantity with respect to nondimensional time.

$$
(\cdot)=\frac{d}{d \tau}, \quad \hat{u}=\frac{u T_{0}}{S_{0}}, \quad \hat{p}=p T_{0}, \quad \hat{g}=\frac{g T_{0}^{2}}{S_{0}}, \quad \hat{F}_{x}=\frac{F_{x} T_{0}^{2}}{m_{0} S_{0}}, \quad \hat{\mathscr{L}}=\frac{\mathscr{L} T_{0}^{2}}{m_{0} S_{0}^{2}}, \quad \hat{I}_{\mathrm{x}}=\frac{I_{\mathrm{x}}}{m_{0} S_{0}^{2}}
$$

The remaining nondimensional variables are computed in a similar fashion. Henceforth, for convenience, the overhats $\hat{\bullet}$ are dropped with the understanding that terms and equations are nondimensional unless specified otherwise.

\subsection{Small Perturbation Equations}

The operator $\nabla_{\zeta}(.)_{\mathrm{n}}$ computes the gradient of the scalar it acts on, with respect to the state vector $\zeta$, at the nominal condition. In the following work, whenever $\nabla_{\zeta}(.)_{\mathrm{n}}$ acts on a vector, we compute it by its action on the corresponding scalars, resulting in a tensor of appropriate dimensions. Let $\boldsymbol{U}=\left(\boldsymbol{\delta}_{a}, \boldsymbol{\delta}_{e}, \boldsymbol{\delta}_{r}\right)^{\top}$ denote the control vector. Let $\boldsymbol{\zeta}_{\mathrm{n}}, \boldsymbol{U}_{\mathrm{n}}$ be the nominal state and control vectors respectively and $\boldsymbol{\zeta}_{p}, \boldsymbol{U}_{p}$ the perturbations in the state and control vectors respectively, from the nominal value. Then, $\nabla_{\boldsymbol{\zeta}}(.)_{\mathrm{n}}=$ $\left.\frac{\partial(.)}{\partial \zeta}\right|_{\zeta=\zeta_{\mathrm{n}}, U=U_{\mathrm{n}}}$. Similarly $\nabla_{\boldsymbol{U}}(.)_{\mathrm{n}}=\left.\frac{\partial(.)}{\partial \boldsymbol{U}}\right|_{\zeta=\zeta_{\mathrm{n}}, U=U_{\mathrm{n}}}$. The perturbed equations, to the first order, are given by

$$
\begin{aligned}
\dot{\boldsymbol{\phi}}_{p} & =\nabla_{\boldsymbol{\zeta}}\left(\boldsymbol{L}_{\mathrm{IB}}(\boldsymbol{\phi}, \boldsymbol{\theta}) \boldsymbol{\omega}\right)_{\mathrm{n}} \cdot \boldsymbol{\zeta}_{p}+\nabla_{U}\left(\boldsymbol{L}_{\mathrm{IB}}(\boldsymbol{\phi}, \boldsymbol{\theta}) \boldsymbol{\omega}\right)_{\mathrm{n}} \cdot U_{p} \\
\dot{\boldsymbol{p}}_{p} & =\nabla_{\boldsymbol{\zeta}}\left(\boldsymbol{p} \times \boldsymbol{\omega}+m g \boldsymbol{R}_{\mathrm{BI}}(\boldsymbol{\phi}, \boldsymbol{\theta}, \psi) \boldsymbol{e}_{3}+\boldsymbol{F}_{\mathrm{visc}}\right)_{\mathrm{n}} \cdot \boldsymbol{\zeta}_{p}+\nabla_{U}\left(\boldsymbol{p} \times \boldsymbol{\omega}+m g \boldsymbol{R}_{\mathrm{BI}}(\boldsymbol{\phi}, \theta, \psi) \boldsymbol{e}_{3}+\boldsymbol{F}_{\mathrm{visc}}\right)_{\mathrm{n}} \cdot U_{p} \\
\dot{\boldsymbol{h}}_{p} & =\nabla_{\boldsymbol{\zeta}}\left(\boldsymbol{h} \times \boldsymbol{\omega}+\boldsymbol{T}_{\mathrm{visc}}\right)_{\mathrm{n}} \cdot \boldsymbol{\zeta}_{p}+\nabla_{U}\left(\boldsymbol{h} \times \boldsymbol{\omega}+\boldsymbol{T}_{\mathrm{visc}}\right)_{\mathrm{n}} \cdot U_{p}
\end{aligned}
$$

On simplifying (6.3) we get:

$$
\dot{\zeta}_{p}=A \zeta_{p}+B U_{p}
$$

Here $\boldsymbol{A} \in \mathbb{R}^{9 \times 9}$ and $\boldsymbol{B} \in \mathbb{R}^{9 \times 3}$, and they are respectively given by:

$$
\begin{aligned}
& \boldsymbol{A}=\left(\begin{array}{c}
\nabla_{\boldsymbol{\zeta}}\left(\boldsymbol{L}_{\mathrm{IB}}(\boldsymbol{\phi}, \boldsymbol{\theta}) \boldsymbol{\omega}\right) \\
\nabla_{\boldsymbol{\zeta}}\left(\boldsymbol{p} \times \boldsymbol{\omega}+m g \boldsymbol{R}_{\mathrm{BI}}(\boldsymbol{\phi}, \boldsymbol{\theta}, \psi) \boldsymbol{e}_{3}+\boldsymbol{F}_{\mathrm{visc}}\right) \\
\nabla_{\boldsymbol{\zeta}}\left(\boldsymbol{h} \times \boldsymbol{\omega}+\boldsymbol{T}_{\mathrm{visc}}\right)
\end{array}\right)_{\mathrm{n}} \\
& \boldsymbol{B}=\left(\begin{array}{c}
0_{9 \times 3} \\
\nabla_{U} \boldsymbol{F}_{\text {visc }} \\
\nabla_{U} \boldsymbol{T}_{\mathrm{visc}}
\end{array}\right)_{\mathrm{n}}
\end{aligned}
$$




\subsection{Stability of the Nominal Solution}

The nominal nondimensional state used in this section is

$$
x_{\mathrm{n}}=(-0.2972,-0.8671,11.1393,-5.0600,8.7169,4.7906,-1.1905,3.8873)^{\mathrm{T}}
$$

The nominal controls are $\delta_{a}=-8^{\circ}, \delta_{e}=20^{\circ}$, and $\delta_{r}=-21^{\circ}$.

The eigenvalues of the state matrix $A$ for dynamic equations linearized around this nominal solution are

$$
\begin{array}{ll}
0 & \\
-2.6526+8.9534 i & -2.6526-8.9534 i \\
-0.3384+6.4586 i & -0.3384-6.4586 i \\
-2.0803+5.3882 i & -2.0803-5.3882 i \\
-0.7994+0.0000 i & -2.2604+0.0000 i
\end{array}
$$

The right eigenvector corresponding to the zero eigenvalue is $(0,0,1,0,0,0,0,0,0)^{\top}$, corresponding to motion in the $\psi$ direction. In the nominal state, $\psi$ increases steadily in time: $\psi(t)=\left(\dot{\psi}_{\mathrm{n}}+\delta \dot{\psi}(t)\right) t$.

The dynamic equations are nonlinear and the system exhibits many neighboring steady spin solutions, as shown in the bifurcation diagram for the GTM in Ref. [41]. One of the challenges in formulating a control law, therefore, is to ensure that the system does not converge to a different steady motion.

\subsection{Region of Attraction Around the Nominal Solution}

The region of attraction around the chosen reference equilibrium solution is analyzed via Monte-Carlo simulations. We determine an approximate value of the maximum percentage of perturbations in the state variables, with the controls held constant at the nominal value, above which the system diverges. For the equilibrium chosen in this chapter, a maximum of $40 \%$ perturbations can be applied to the state variables before they diverge. The angle $\phi$ is the first to diverge as evident from the eigenvector corresponding to the eigenvalues with the largest real part after $0--0.3384+6.4586 i,-0.3384-6.4586 i$. The eigenvector corresponding to $-0.3384+6.4586 i$, is 


$$
\begin{gathered}
0.5903+\quad 0.0000 i \\
-0.0103+0.3881 i \\
-0.4366-0.0205 i \\
0.2294-0.1342 i \\
0.3190-\quad 0.0438 i \\
-0.0913+0.2176 i \\
0.0555+\quad 0.0898 i \\
-0.1373-0.2003 i \\
0.0961-\quad 0.0207 i
\end{gathered}
$$

Therefore, the largest component of the eigenvalue is in the direction of $\phi$. The simulation results are shown in fig. 6.1.

\subsection{Formulation}

Matrices $\boldsymbol{A}$ and $\boldsymbol{B}$ are given by the perturbed equations developed in (6.5). Let $\boldsymbol{\eta}=\boldsymbol{R}_{\mathrm{IW}} \boldsymbol{e}_{1}$, be the output we are interested in. The vector $\boldsymbol{\eta}$ denotes the direction of the velocity vector in the inertial frame. The rotation matrix from the wind to the inertial frame is defined as: $\boldsymbol{R}_{\mathrm{IW}}=\boldsymbol{R}_{\mathrm{IB}} \boldsymbol{R}_{\mathrm{BW}}$. Following an approach similar to that in section 6.2 , the perturbed equation for $\eta$ to the first order is given by:

$$
\dot{\boldsymbol{\eta}}_{p}=\nabla_{\boldsymbol{\zeta}}\left(\boldsymbol{R}_{\mathrm{IB}}(\phi, \theta, \psi) \boldsymbol{R}_{\mathrm{BW}}(\alpha, \beta) \boldsymbol{e}_{1}\right)_{\mathrm{n}} \cdot \boldsymbol{\zeta}_{p}+\nabla_{\boldsymbol{U}}\left(\boldsymbol{R}_{\mathrm{IB}}(\phi, \theta, \psi) \boldsymbol{R}_{\mathrm{BW}}(\alpha, \beta) \boldsymbol{e}_{1}\right)_{\mathrm{n}} \cdot \boldsymbol{U}_{p}
$$

The above equation simplifies to

$$
\dot{\eta}_{p}=\boldsymbol{C}(t) \cdot \zeta_{p}
$$

where

$$
\boldsymbol{C}(t)=\nabla_{\boldsymbol{\zeta}}\left(\boldsymbol{R}_{\mathrm{IB}}(\phi, \theta, \psi) \boldsymbol{R}_{\mathrm{BW}}(\alpha, \beta) \boldsymbol{e}_{1}\right)_{\mathrm{n}}
$$

Matrix $\boldsymbol{C}(t)$ is a function of $\psi_{\mathrm{n}}=\dot{\psi}_{\mathrm{n}} t$, and so is periodic with a period $T=\frac{2 \pi}{\dot{\psi}_{\mathrm{n}}}$.

Let the integrated perturbed output be $\boldsymbol{\varepsilon}_{p}(t)=\int_{0}^{t} \eta_{p}(\tau) d \tau$. So the complete system considered here, similar to that in (5.1), is given by: 

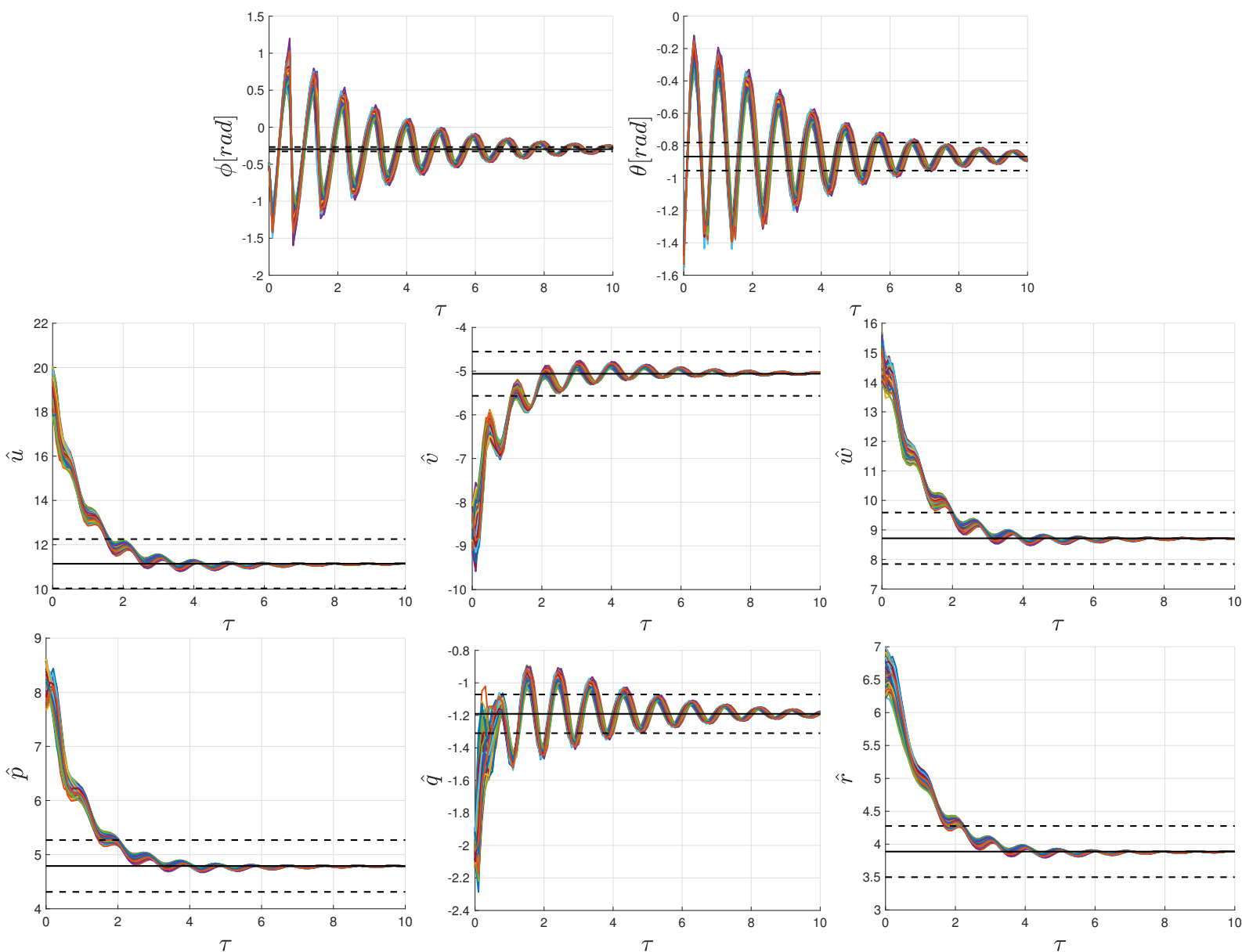

Figure 6.1: Estimation of the region of attraction: Monte-Carlo simulation results, when a maximum of 40 $\%$ perturbation is applied to the state variable about the reference spin equilibrium. The solid black line is the nondimensional nominal state and the dashed black line is at a $10 \%$ perturbation from the nominal value.

$$
S 2=\left\{\begin{array}{l}
\dot{\boldsymbol{\zeta}}_{p}(t)=\boldsymbol{A} \boldsymbol{\zeta}_{p}(t)+\boldsymbol{B} \boldsymbol{U}_{p}(t), \quad \boldsymbol{\zeta}_{p}\left(t_{0}\right)=\boldsymbol{\zeta}_{p 0} \\
\boldsymbol{\eta}_{p}(t)=\boldsymbol{C}(t) \boldsymbol{\zeta}_{p}(t) \\
\boldsymbol{\varepsilon}_{p}(t)=\int_{t_{0}}^{t} \boldsymbol{\eta}_{p}(\tau) d \tau
\end{array}\right.
$$

From now on, the $p$ in the subscript is dropped with the understanding that the quantities considered are still the perturbed quantities. Note that system $S 2$ in (6.8) is a special case of system $S 1$ in (5.1).

We seek to direct the average inertial velocity direction per time-period $T$, given by $\boldsymbol{\varepsilon}(T)$, to a desired value $\boldsymbol{\varepsilon}_{d}$. The constant control input applied for a time $\Delta T$ every cycle, to drive $\boldsymbol{\varepsilon}(T)$ to $\boldsymbol{\varepsilon}_{d}$ is given by (5.6).

Remark 4. Note that once the system is perturbed, $\psi$ may take less or more time than $T$ to complete a cycle from 0 to $2 \pi$. In that case, $T$ has to be replaced by this new time $T^{\prime}$ in calculating the control input. 
Correspondingly $\bar{C}, \bar{D}, \Delta T$ will change to $\bar{C}^{\prime}, \bar{D}^{\prime}, \Delta T^{\prime}$ also.

\subsection{Simulation Results}

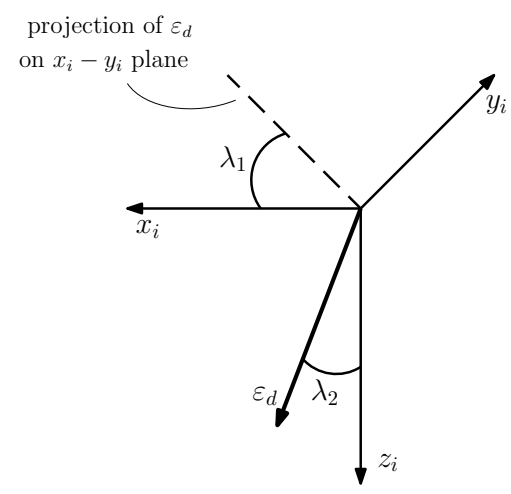

Figure 6.2: Schematic to show $\boldsymbol{\varepsilon}_{d}$.

We have formulated a control strategy of the form $u_{0}=\bar{D}^{-1}\left(\boldsymbol{\varepsilon}_{d}(T)-\bar{C} \boldsymbol{\zeta}_{0}\right)$. Considering the given nominal steady motion for the GTM and taking $n=10$, so that the input is applied over one-tenth of a helical revolution. The desired direction $\boldsymbol{\varepsilon}_{\mathrm{d}}$ is defined in terms of two angles: $\theta_{1}$ from the inertial $x$ direction and $\theta_{2}$ from the inertial $z$ direction. So $\boldsymbol{\varepsilon}_{\mathrm{d}}$ is defined as:

$$
\boldsymbol{\varepsilon}_{\mathrm{d}}=\left(\cos \lambda_{1} \sin \lambda_{2}, \sin \lambda_{1} \sin \lambda_{2}, \cos \lambda_{2}\right)
$$

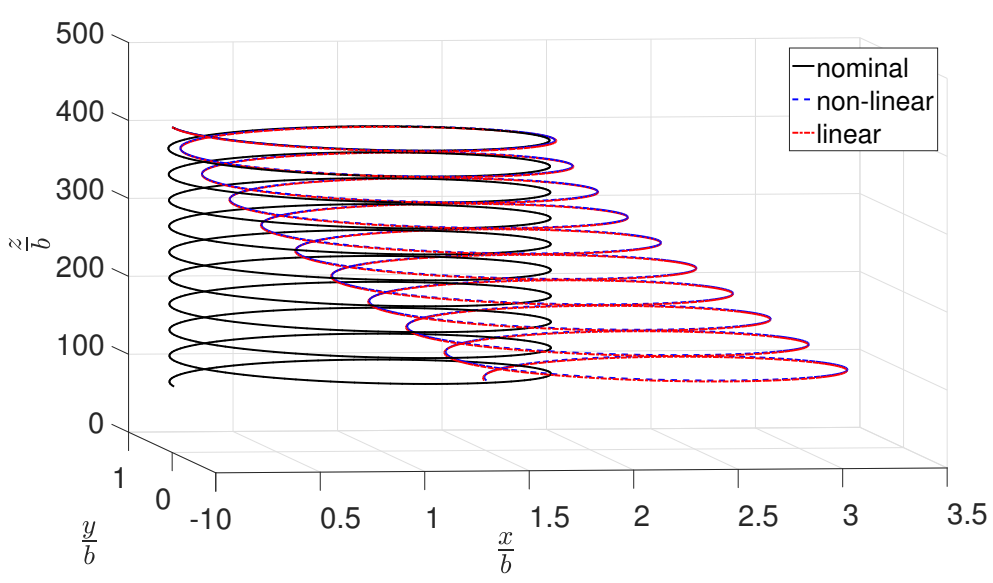

Figure 6.3: The nominal trajectory of an aircraft in a steep vertical spin is shown in black. The controlled trajectory of an aircraft to direct it along $\theta_{1 \mathrm{~d}}=10^{\circ}$ and $\theta_{2 \mathrm{~d}}=0.1^{\circ}$ is demonstrated by a nonlinear model in blue and by a linear model in red. 
Figure 6.3 shows the nominal trajectory and the controlled trajectory for an aircraft with $\lambda_{1}=10^{\circ}$ and $\lambda_{2}=0.1^{\circ}$. The simulation was run for $\tau=100$ in each case. The error in the desired cycle averaged direction of the inertial velocity is defined as:

$$
e_{\lambda_{1}}=\lambda_{1 \mathrm{w}}-\lambda_{1 \mathrm{~g}}
$$

where $\lambda_{1 \mathrm{w}}$ is the wanted $\lambda_{1}$ in degrees and $\lambda_{1 \mathrm{~g}}$ is the value of $\lambda_{1}$ we get via after the linear control is applied to a nonlinear simulation. For $\lambda_{1}=10^{\circ}$ and $\lambda_{2}=0.1^{\circ}$, the error in $\lambda_{1}$ by a nonlinear simulation is $5.5116^{\circ}$.

\subsection{Error Analysis}

Figures 6.4-6.6 show the variation of $\lambda_{1 \mathrm{~g}}$ with the desired azimuthal direction $\left(\lambda_{1 \mathrm{w}}\right)$, across varying desired inclination angles from the $z$ axis $\left(\lambda_{2 \mathrm{w}}\right)$, and varying times $\Delta T$ for which the constant control is applied. Each case was run for $\tau=10$, and the control inputs were bounded at $\pm 15^{\circ}$ from the nominal value. However, it must be noted that the linear assumption if valid for the control inputs varying less than $\pm 10^{\circ}$ from the nominal value.
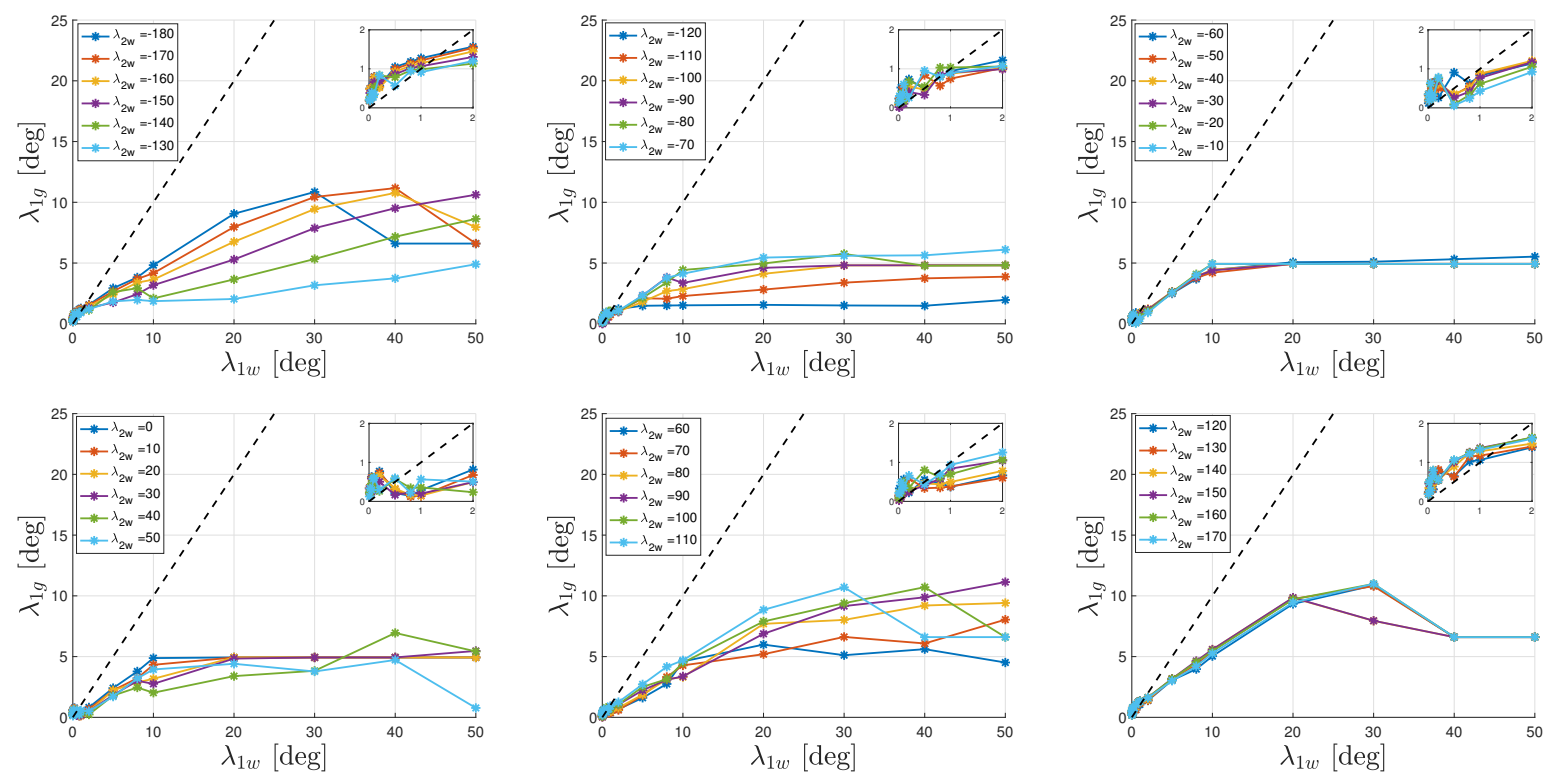

Figure 6.4: Variation of the achieved inclination angle $\lambda_{1 \mathrm{~g}}$ with varying desired inclination angles $\lambda_{1 \mathrm{w}}$ and desired azimuth angles $\lambda_{2 \mathrm{w}}$. Here the time interval for which the constant control is applied $\Delta T$ is $\frac{1}{5}$. The dashed black line is a slope 1 line for reference.

Each figure has an inset figure in it to show the variation of $\lambda_{1 \mathrm{~g}}$ for $\lambda_{1 \mathrm{w}}$ less than $2^{\circ}$. The errors are less than $1^{\circ}$ for $\lambda_{1 \mathrm{w}}$ less than $1^{\circ}$. The error in $\lambda_{1 \mathrm{~g}}$ increases with an increase in $\lambda_{1 \mathrm{w}}$. 

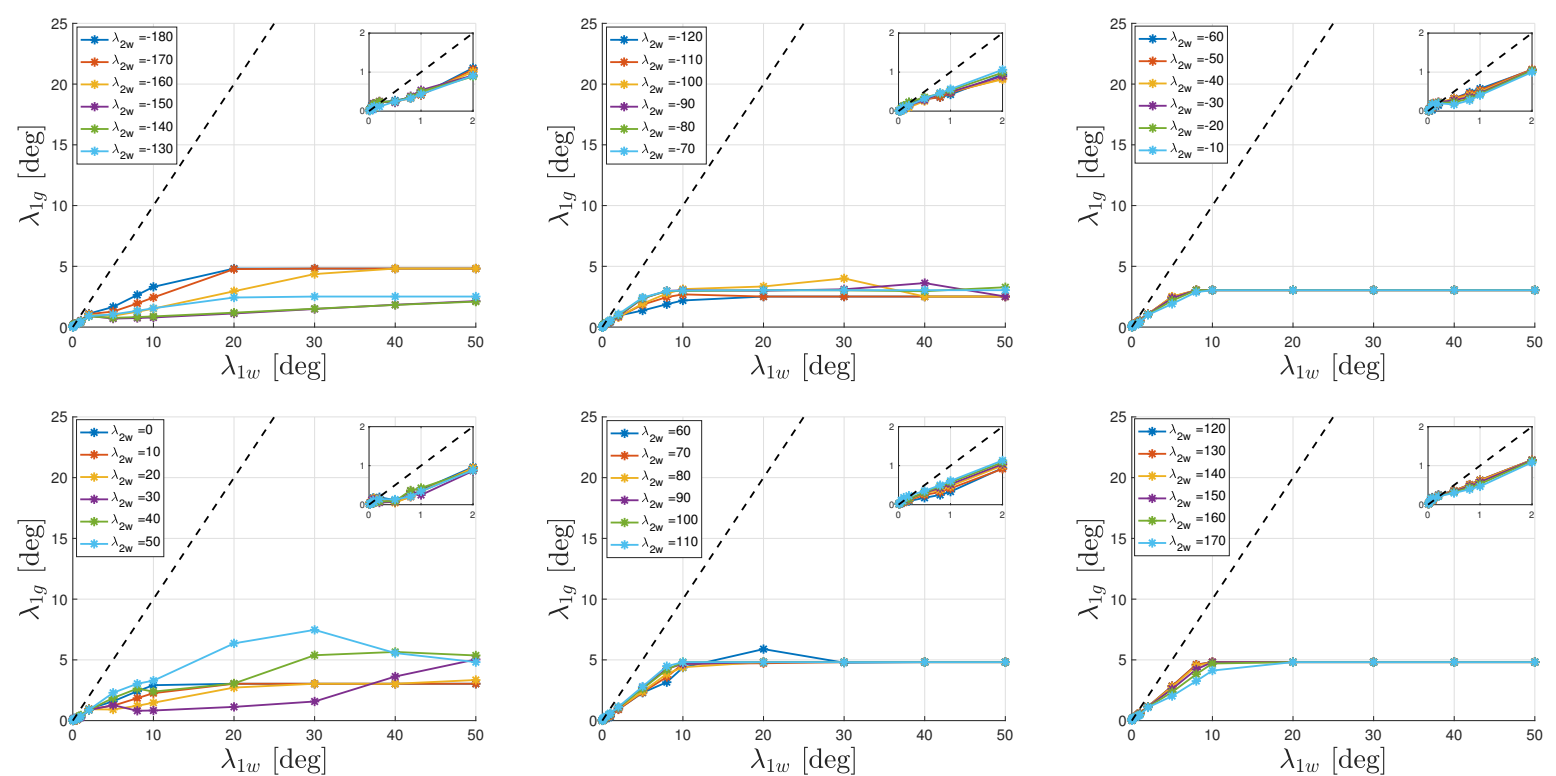

Figure 6.5: Variation of the achieved inclination angle $\lambda_{1 \mathrm{~g}}$ with varying desired inclination angles $\lambda_{1 \mathrm{w}}$ and desired azimuth angles $\lambda_{2 \mathrm{w}}$. Here the time interval for which the constant control is applied $\Delta T$ is $\frac{1}{10}$. The dashed black line is a slope 1 line for reference.
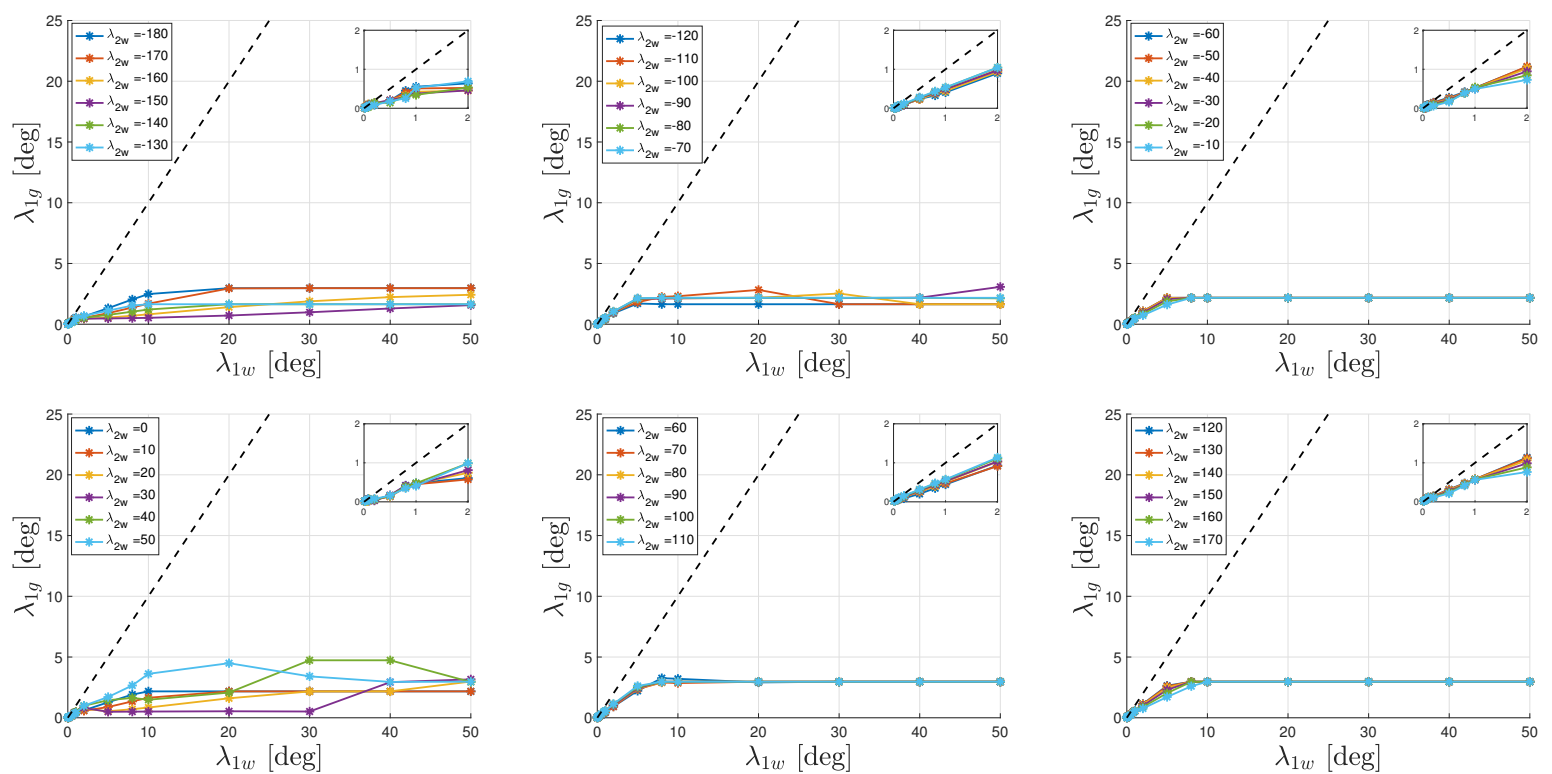

Figure 6.6: Variation of the achieved inclination angle $\lambda_{1 \mathrm{~g}}$ with varying desired inclination angles $\lambda_{1 \mathrm{w}}$ and desired azimuth angles $\lambda_{2 \mathrm{w}}$. Here the time interval for which the constant control is applied $\Delta T$ is $\frac{1}{15}$. The dashed black line is a slope 1 line for reference. 
The figures show that the maximum $\lambda_{1 \mathrm{~g}}$ achieved decreases with a decrease in $\Delta T$. It is about $11^{\circ}$ for $\Delta T=\frac{1}{5}$ and about $3^{\circ}$ for $\Delta T=\frac{1}{15}$. The error in $\lambda_{1}$ increases with a decrease in $\Delta T$. Also the error is lower for azimuth angles close to $180^{\circ}$.

Figures 6.7 - 6.9 show the variation in the achieved azimuth angle $\lambda_{2 \mathrm{~g}}$ with varying desired azimuth angles $\lambda_{2 \mathrm{w}}$, the desired inclination angles $\lambda_{1 \mathrm{w}}$ and the time interval over which the constant control input is applied $\Delta T$.

From the figures, it is evident that the full range of azimuth angles can be achieved. The errors in the achieved azimuth angle $\lambda_{2 \mathrm{w}}$ are large for desired inclination angles $\lambda_{1 \mathrm{w}}$ less than $1^{\circ}$. The errors for $\lambda_{1 \mathrm{w}}<1^{\circ}$ decrease with a decrease in $\Delta T$, but are almost equal for $\lambda_{1 \mathrm{w}}>1^{\circ}$. The errors are larger for azimuth angles close to $0^{\circ}$ and $180^{\circ}$.
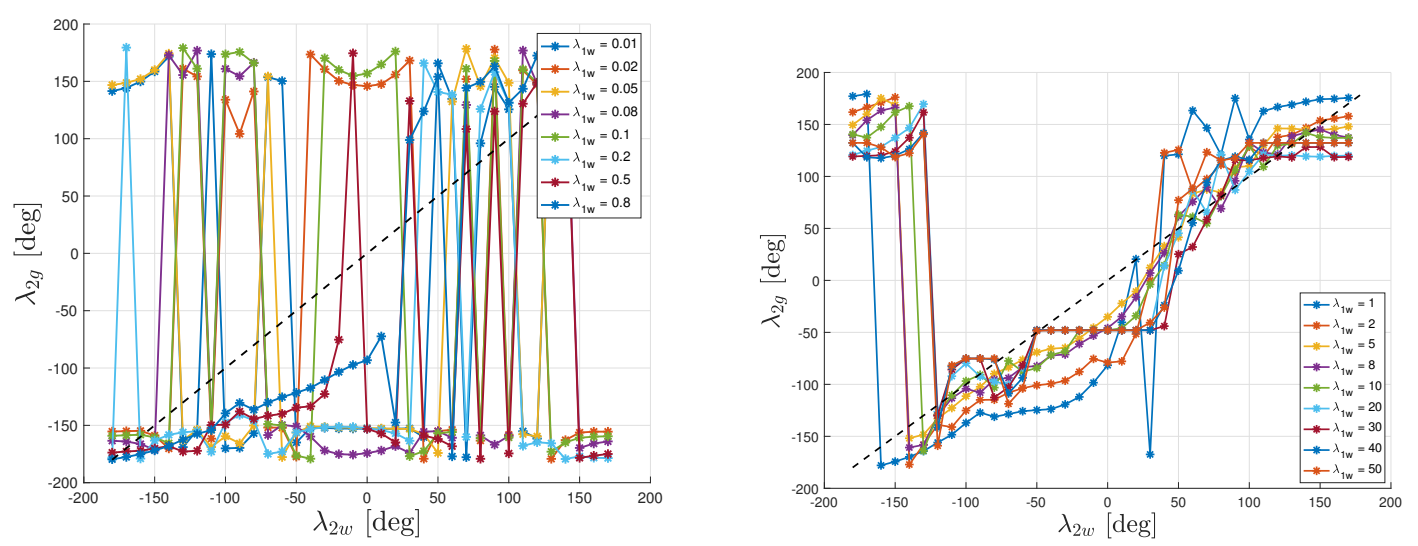

Figure 6.7: Variation of the achieved azimuth angle $\lambda_{2 \mathrm{~g}}$ with varying desired inclination angles $\lambda_{1 \mathrm{w}}$ and desired azimuth angles $\lambda_{2 w}$. Here the time interval for which the constant control is applied $\Delta T$ is $\frac{1}{5}$. The dashed black line is a slope 1 line for reference.

The errors in $\lambda_{1 \mathrm{~g}}$ and $\lambda_{2 \mathrm{~g}}$ result from mainly one of the following two reasons: (1) there is not enough control authority because the control deflections are close to their limiting values or (2) the perturbations in the state are too large and the linear assumption is no longer valid.

Interestingly, there is a trade-off between lower errors in $\lambda_{1 \mathrm{~g}}$ and $\lambda_{2 \mathrm{~g}}$. The errors in $\lambda_{1 \mathrm{~g}}$ seem to be because of not enough control authority, therefore for larger $\Delta T$ the errors are smaller. Whereas the errors in $\lambda_{2 \mathrm{~g}}$ seem to be because large perturbations and so the performance is poor for larger $\Delta T$.

Another trade-off is that a larger $\lambda_{1 \mathrm{w}}$ results in a larger component of the velocity in the $x-y$ plane and therefore faster changes in the cycle averaged inertial position of the aircraft projected onto the $x-y$ plane. However, this also produces larger uncertainties in the resulting $\lambda_{1 \mathrm{~g}}$. 

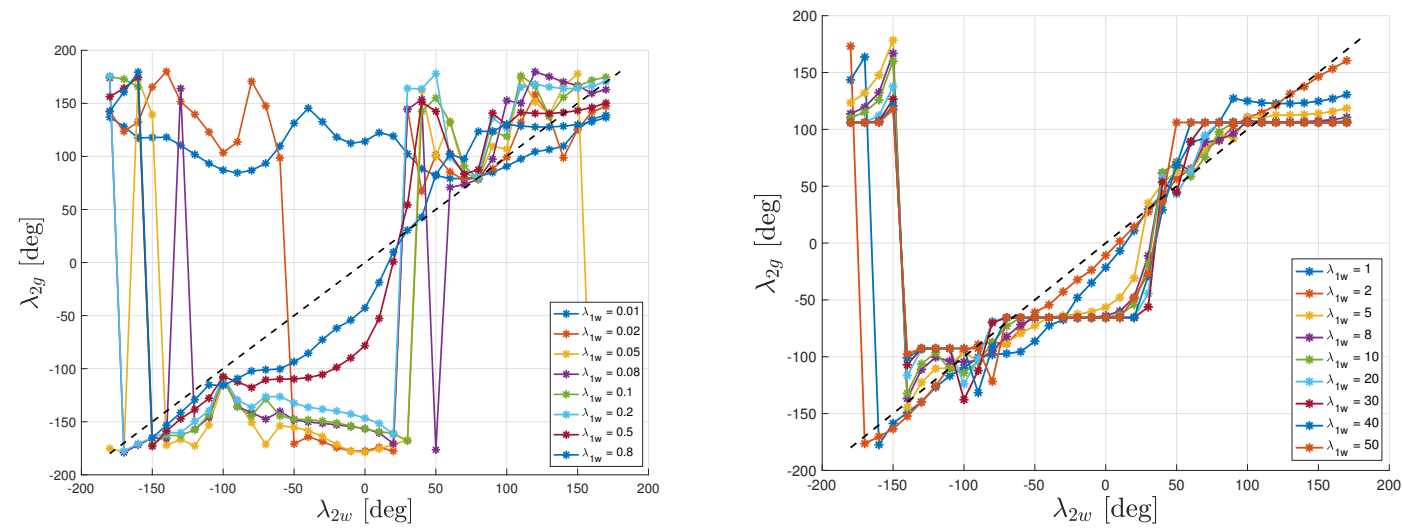

Figure 6.8: Variation of the achieved azimuth angle $\lambda_{2 \mathrm{~g}}$ with varying desired inclination angles $\lambda_{1 \mathrm{w}}$ and desired azimuth angles $\lambda_{2 \mathrm{w}}$. Here the time interval for which the constant control is applied $\Delta T$ is $\frac{1}{10}$. The dashed black line is a slope 1 line for reference.
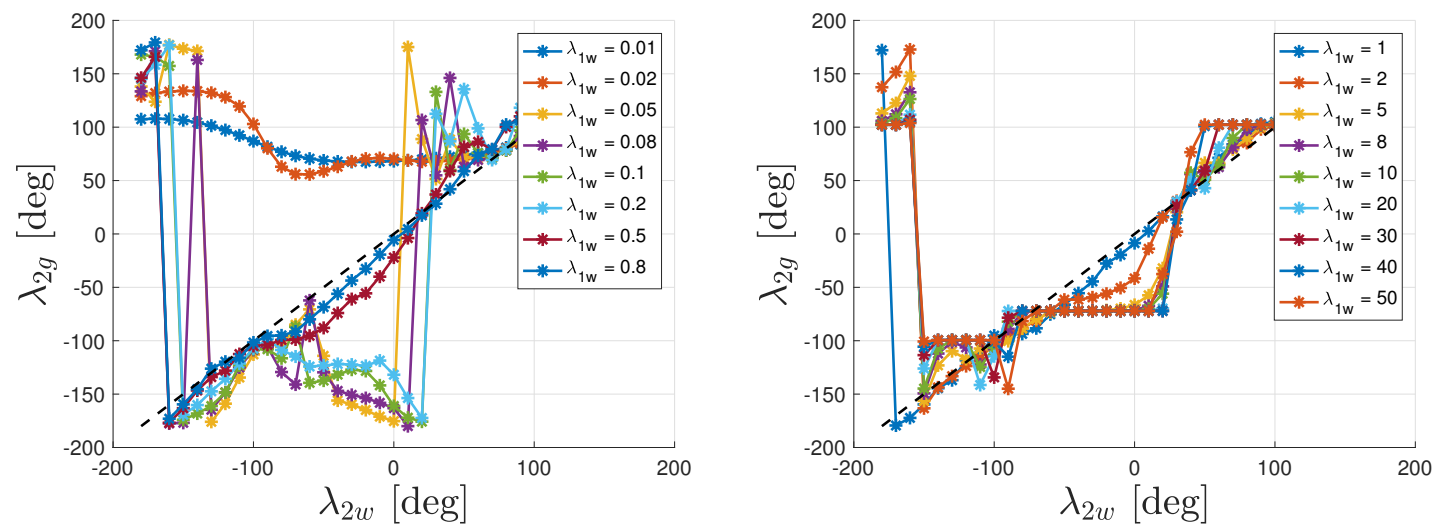

Figure 6.9: Variation of the achieved azimuth angle $\lambda_{2 \mathrm{~g}}$ with varying desired inclination angles $\lambda_{1 \mathrm{w}}$ and desired azimuth angles $\lambda_{2 \mathrm{w}}$. Here the time interval for which the constant control is applied $\Delta T$ is $\frac{1}{15}$. The dashed black line is a slope 1 line for reference. 


\section{Chapter 7}

\section{Conclusions and Future Work}

In this thesis I present a feedback control law that uses pulse width modulation (PWM) to direct the cycleaveraged inertial velocity vector of an aircraft in an unpowered, spinning descent. The linear control law is demonstrated in simulation for a nonlinear dynamical model of the GTM described in [23]. Errors in the desired azimuth and inclination angles are studied.

With the control law formulated, the entire range of azimuth angles can be achieved, whereas the maximum inclination angle was about $11^{\circ}$ and it decreased significantly with a decrease in $\Delta T$. The main sources of errors are the lack of control authority and large perturbations in the state variables that make the linear assumption invalid. A continuous control law based on an optimal control strategy should be able to circumvent some of these problems. In the context of flight termination, incorporating an outer guidance loop to direct the cycle-averaged heading based on inertial position may provide the ability to target a desired, safe impact location. 


\section{Appendix A}

\section{Aerodynamic Model}

$$
\begin{aligned}
C_{D} & =\theta_{1}+\theta_{2} \alpha+\theta_{3} \alpha \bar{q}+\theta_{4} \alpha \delta_{e}+\theta_{5} \alpha^{2}+\theta_{6} \alpha^{2} \bar{q}+\theta_{7} \alpha^{2} \delta_{e}+\theta_{8} \alpha^{3}+\theta_{9} \alpha^{3} \bar{q}+\theta_{10} \alpha^{4}, \\
C_{Y} & =\theta_{11} \beta+\theta_{12} \bar{p}+\theta_{13} \bar{r}+\theta_{14} \delta_{a}+\theta_{15} \delta_{r}, \\
C_{L} & =\theta_{16}+\theta_{17} \alpha+\theta_{18} \bar{q}+\theta_{19} \delta_{e}+\theta_{20} \alpha \bar{q}+\theta_{21} \alpha^{2}+\theta_{22} \alpha^{3}+\theta_{23} \alpha^{4}, \\
C_{l} & =\theta_{24} \beta+\theta_{25} \bar{p}+\theta_{26} \bar{r}+\theta_{27} \delta_{a}+\theta_{28} \delta_{r}, \\
C_{m} & =\theta_{29}+\theta_{30} \alpha+\theta_{31} \bar{q}+\theta_{32} \delta_{e}+\theta_{33} \alpha \bar{q}+\theta_{34} \alpha^{2} \bar{q}+\theta_{35} \alpha^{2} \delta_{e}+\theta_{36} \alpha^{3} \bar{q}+\theta_{37} \alpha^{3} \delta_{e}+\theta_{38} \alpha^{4}, \\
C_{n} & =\theta_{39} \beta+\theta_{40} \bar{p}+\theta_{41} \bar{r}+\theta_{42} \delta_{a}+\theta_{43} \delta_{r}+\theta_{44} \beta^{2}+\theta_{45} \beta^{3} .
\end{aligned}
$$

Here, $\theta_{i}$ are the fixed numerical coefficients which depend on the aircraft model considered, and nondimensional angular velocites are defined as $-\bar{p}=\frac{b p}{2 V_{0}}, \quad \bar{q}=\frac{c q}{2 V_{0}}, \quad \bar{r}=\frac{b r}{2 V_{0}}$.

The above definition of the coefficients are consistent with Ref. [23]. 


\section{Appendix B}

\section{Plots for Stable Equilibrium Spin Solutions}

We have compiled all the stable equilibrium solutions (except in Fig. B.1, which has unstable solutions also), with the radius of the helix varying from $1-5 \mathrm{~m}$, where the wing span of the aircraft is approximately $2 \mathrm{~m}$.

In Fig. B.1, the red circles are the unstable equilibria and the black dots at the stable equilibria. Every point on the figure corresponds to three control parameters $-\delta_{a}, \delta_{e}, \delta_{r}$. The figure is a 2D projection of the points on the respective control input planes. That is why there are some points with overlapping black dots and red circles corresponding to the same two control inputs plotted, but a different third control input value. The figure shows that the aileron input plays an important role in determining the stability of the equilibrium solution. For $\delta_{a}$ varying from 0 to -20 degrees, all the solutions are stable.
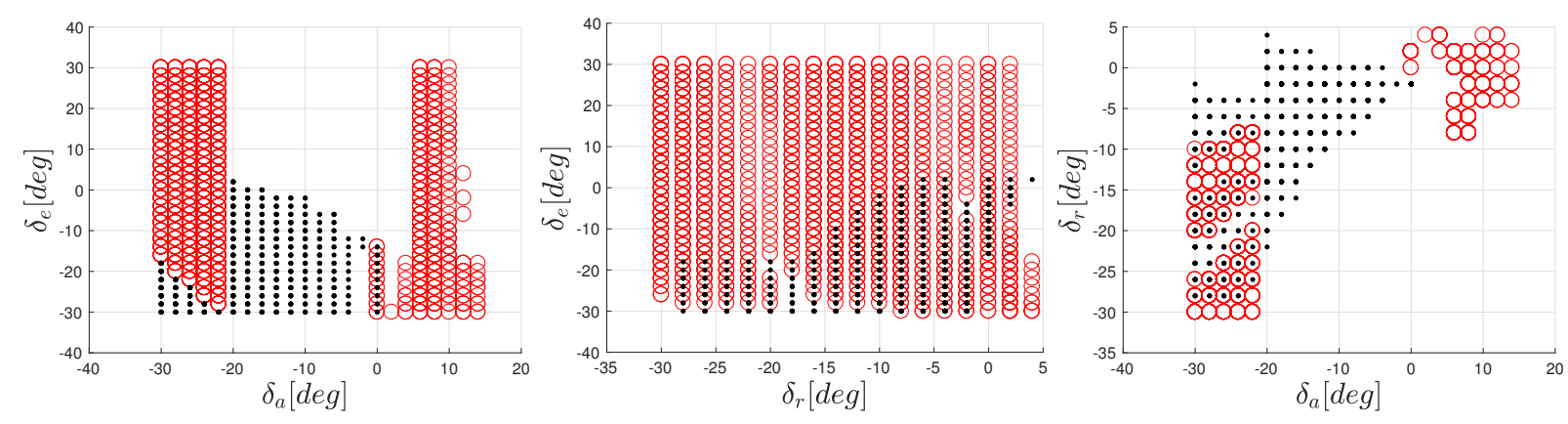

Figure B.1: Relation between the stability of the steady spin equilibria and the control inputs. The solutions are for the radius of the helix varying from $1-5 \mathrm{~m}$. Red circles: unstable equilibria, black dots: stable equilibria.

For the rest of the figures, green dots represent the variation of the variables with an aileron input, blue dots the variation with an elevator input and red dots the variation with a rudder input. Fig. B.2 show the variation of the angles $\phi$ and $\theta$ for stable equilibria with variations in the control inputs. Again each point 
corresponds to a set of three control inputs $-\delta_{a}, \delta_{e}, \delta_{r}$. There seem to be fewer stable equilibria solutions for higher rudder inputs. The angle $\phi$ is in general lower for higher values of the rudder input; there are no clear correlations of $\phi$ with the elevator and aileron inputs. The angle $\theta$ shows a high correlation with the elevator inputs, as would be expected. It is larger for smaller values of the elevator input.
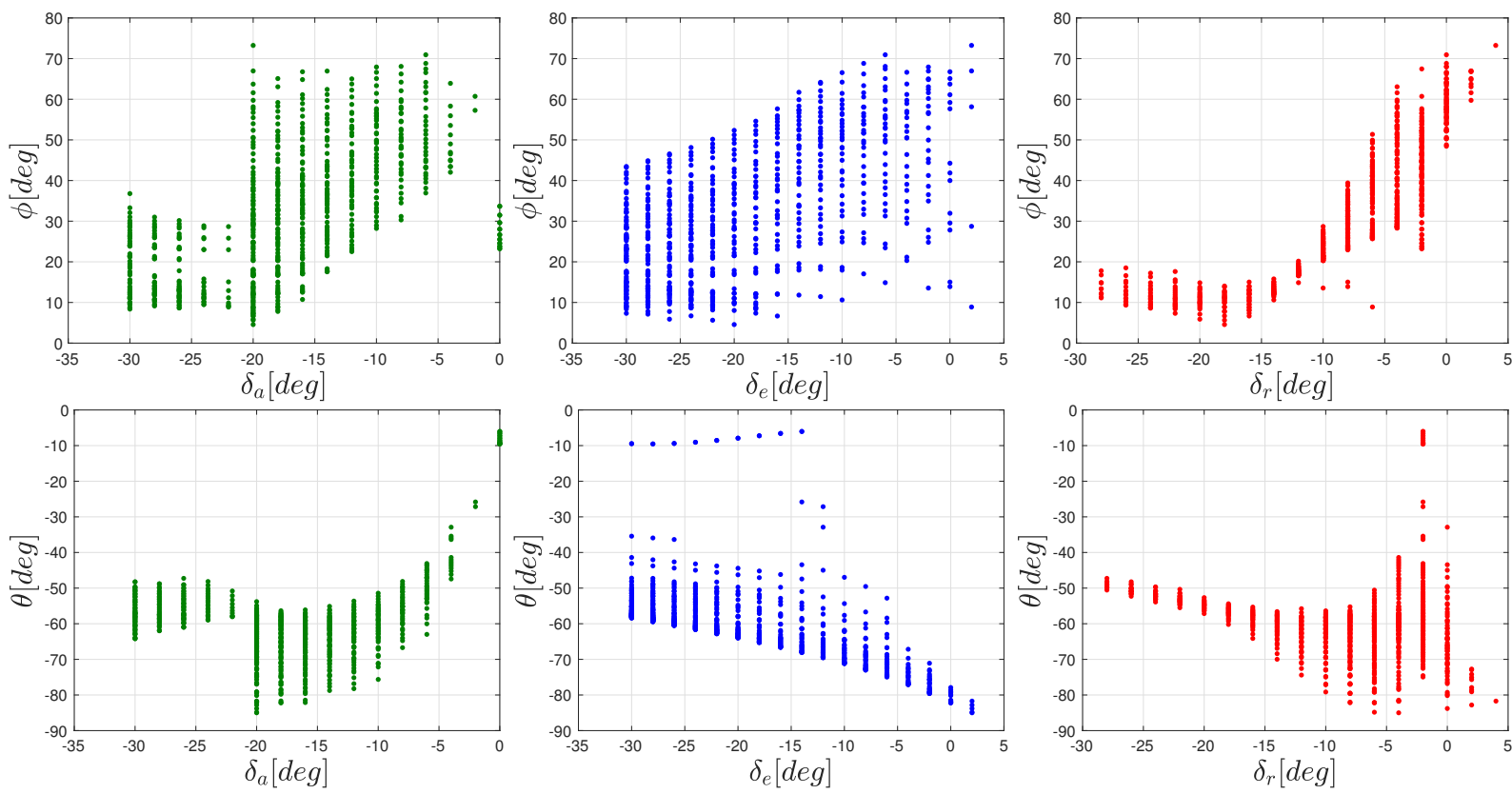

Figure B.2: Variation of $\phi$ and $\theta$ at stable spin equilibria, with the control inputs $\delta_{a}, \delta_{e}$ and $\delta_{r}$. The equilibria are restricted to the radius of the helix varying from $1-5 \mathrm{~m}$.

Fig. B. 3 shows the variation of the body velocities at the stable equilibria with the control inputs. There is a strong correlation between $u$ and $\delta_{e}$. The body-x velocity $u$ increases with a decrease in $\delta_{e}$. The body-z velocity $w$ shows a strong correlation with $\delta_{r}$ and increases with larger rudder deflections to negative angles. The body-y velocity $v$ doesn't show any strong trends with a variation in the equilibrium control inputs.

Fig. B.4 shows the variation of the body angular velocities at the stable spin equilibria with the control inputs. $p$ seems to increase with a decrease in $\delta_{e}, q$ shows no clear trends and $r$ increases with larger deflections in the rudder and aileron controls at negative angles.

Fig. B.5 shows the variation of the body angular speed $\omega$, linear speed $V$ and the 2-norm of the eigenvalues $\|\lambda\|_{2}$ at the stable spin equilibria with the control inputs. The angular speed $\omega$ increases with a decrease in the elevator deflections and with an increase in the rudder and aileron deflections. The angular speed shows a strong correlation with the elevator and is larger for smaller elevator deflections. The norm of the eigenvalue seems to depend the most on the aileron input. It increases with a decrease in the aileron deflection. It is largest for $\delta_{a}=0$. Note that $\omega \sim 0$ for that case, which implies the equilibria are most stable for a close to level flight condition. 

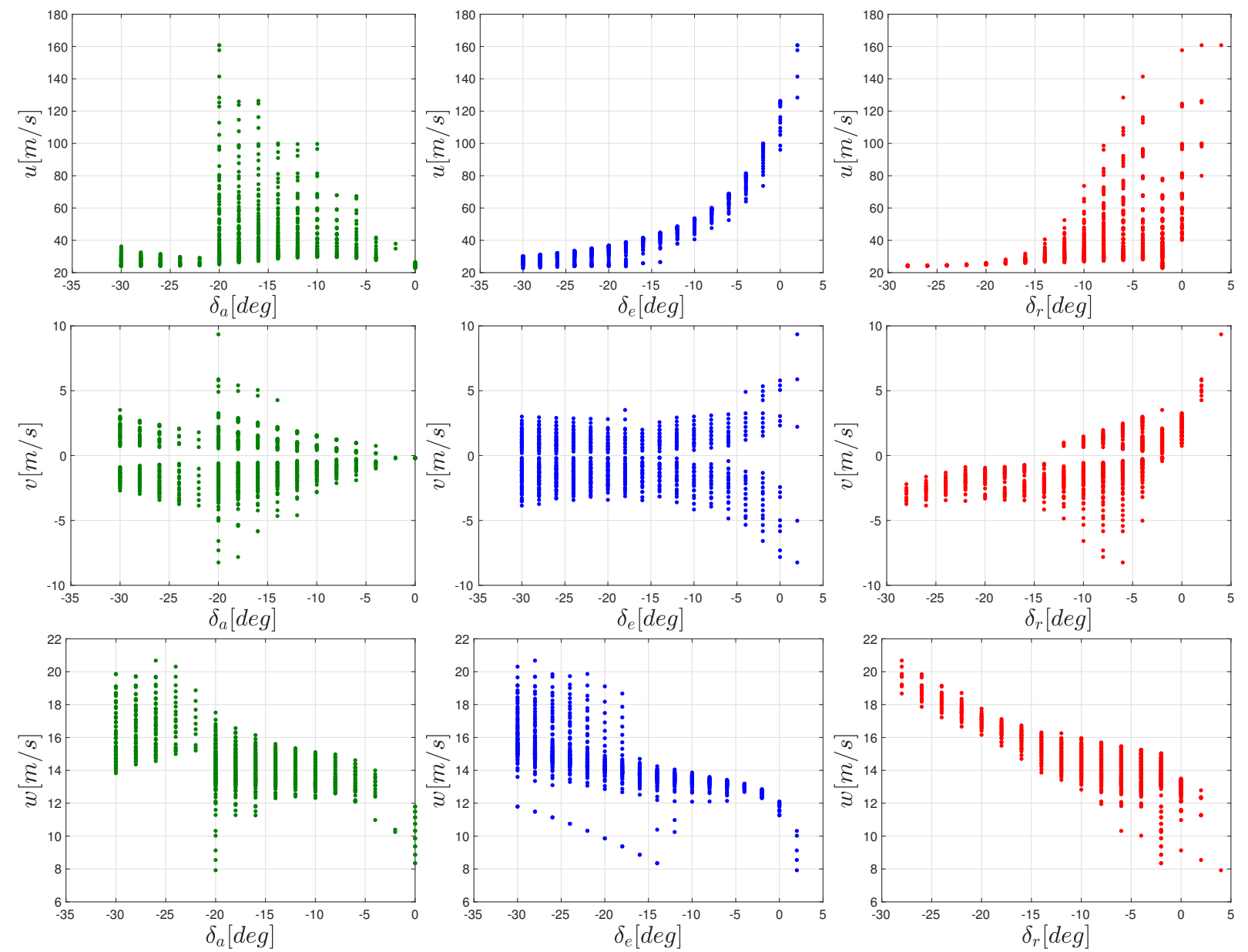

Figure B.3: Variation of the body velocities $u, v$ and $w$ at the stable spin equilibria, with the control inputs $\delta_{a}, \delta_{e}$ and $\delta_{r}$. The equilibria are restricted to the radius of the helix varying from $1-5 \mathrm{~m}$. 

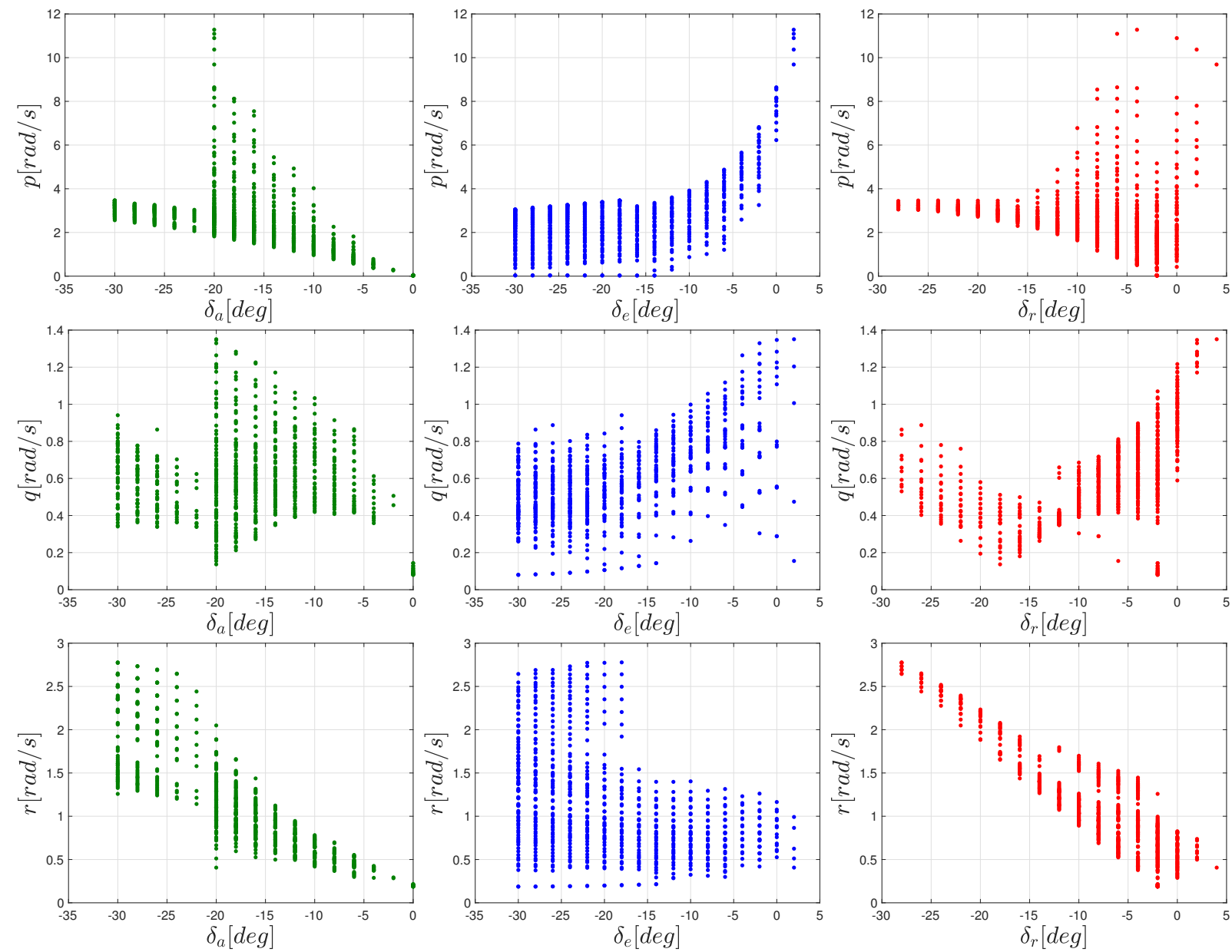

Figure B.4: Variation of the body angular velocities $p, q$ and $r$ at the stable spin equilibria, with the control inputs $\delta_{a}, \delta_{e}$ and $\delta_{r}$. The equilibria are restricted to the radius of the helix varying from $1-5 \mathrm{~m}$. 

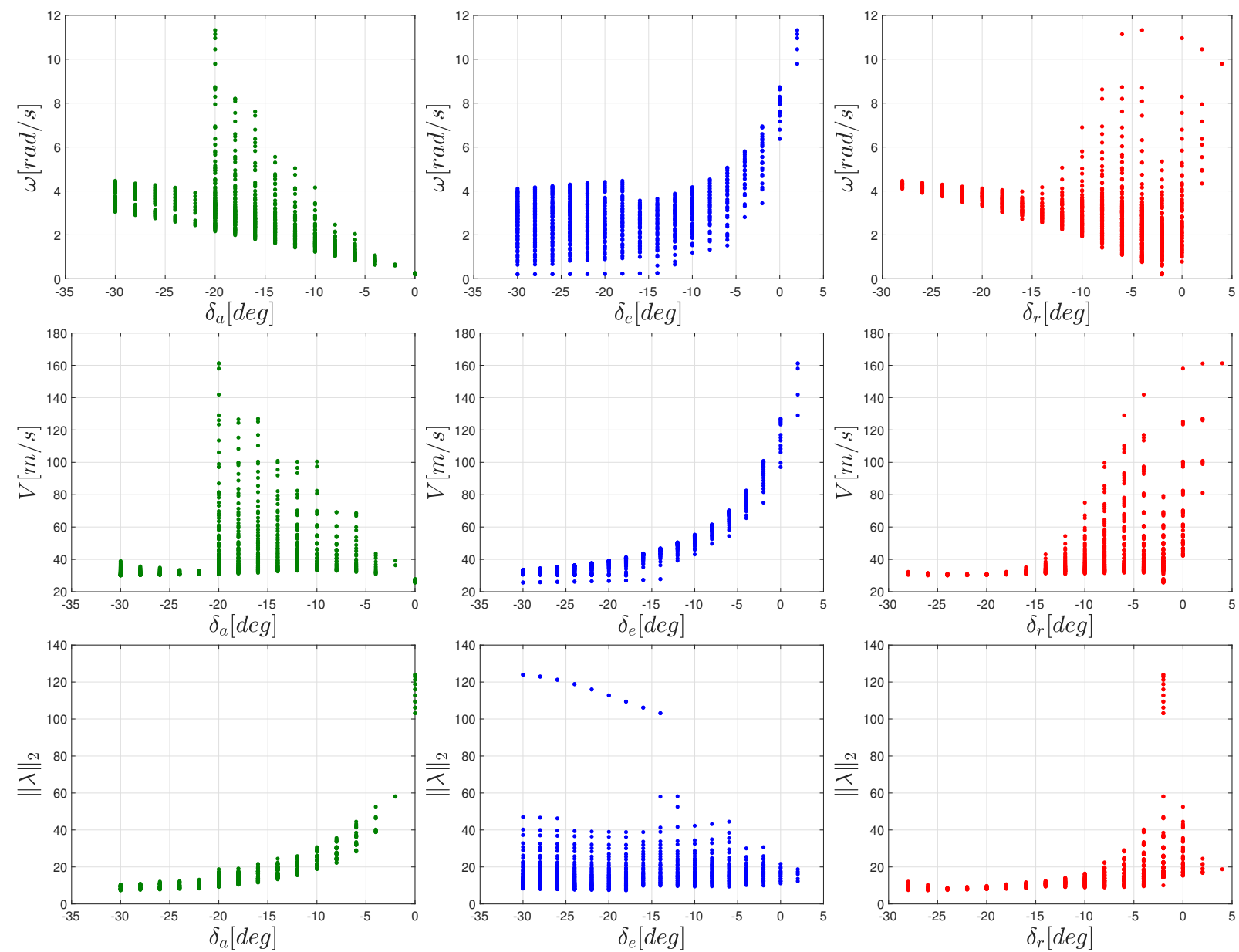

Figure B.5: Variation of the angular speed (which is equal to the yaw rate at equilibrium) $\omega$, the linear speed $V$ and the 2-norm of the eigenvalues $\|\lambda\|_{2}$ with the control inputs $\delta_{a}, \delta_{e}$ and $\delta_{r}$. The equilibria are restricted to the radius of the helix varying from $1-5 \mathrm{~m}$. 


\section{Appendix C}

\section{Plots for Stable and Unstable Equilibrium Spin Solutions}
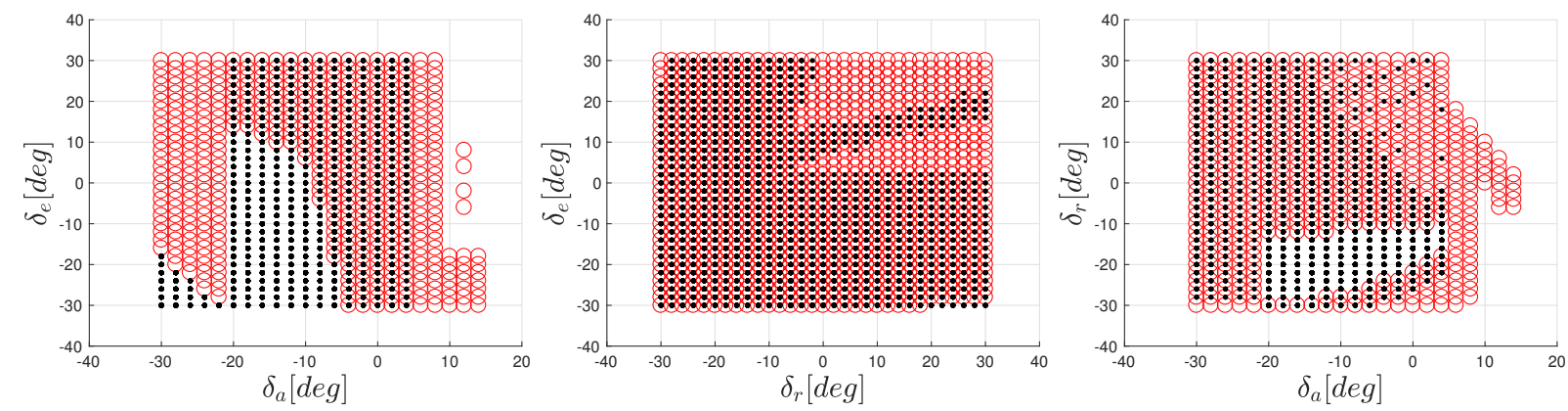

Figure C.1: Relation between the stability of the steady spin equilibria and the control inputs. Red circles: unstable equilibria, black dots: stable equilibria. The radius of the helical descent of the equilibria is restricted to $200 \mathrm{~m}$. 

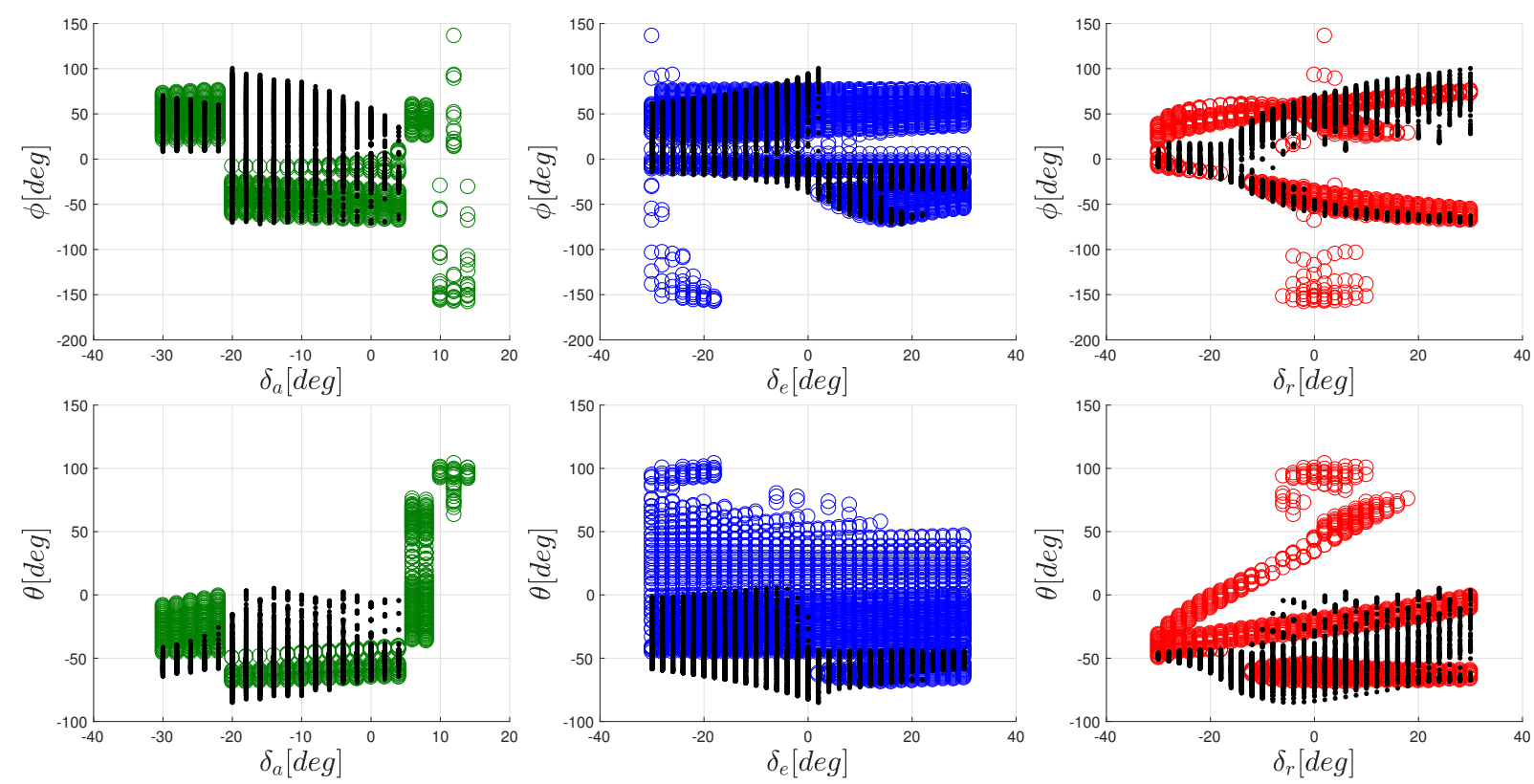

Figure C.2: Variation of $\phi$ and $\theta$ at stable spin equilibria, with the control inputs $\delta_{a}, \delta_{e}$ and $\delta_{r}$. The radius of the helical descent of the equilibria is restricted to $200 \mathrm{~m}$. 

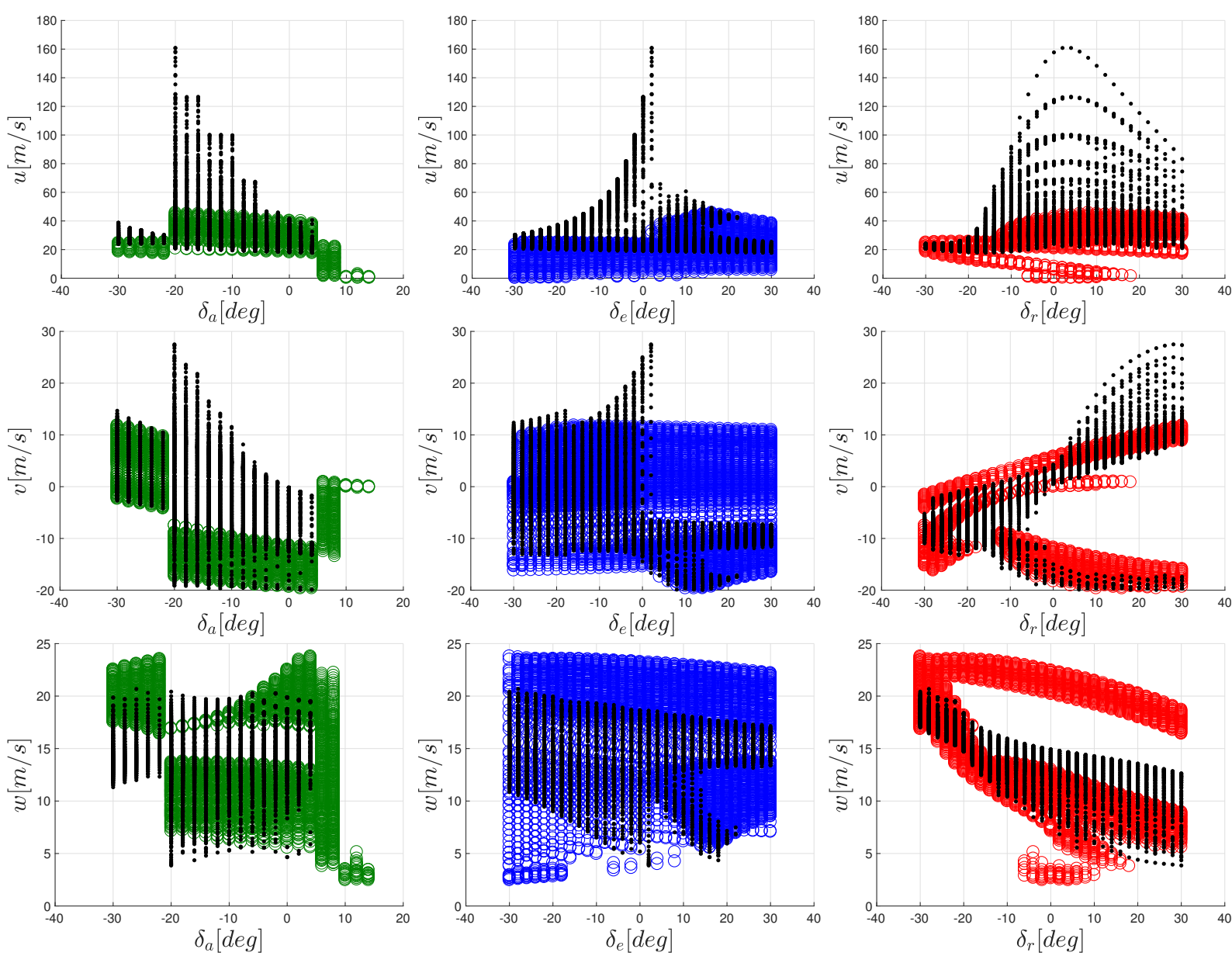

Figure C.3: Variation of the body velocities $u, v$ and $w$ at the stable spin equilibria, with the control inputs $\delta_{a}, \delta_{e}$ and $\delta_{r}$. The radius of the helical descent of the equilibria is restricted to $200 \mathrm{~m}$. 

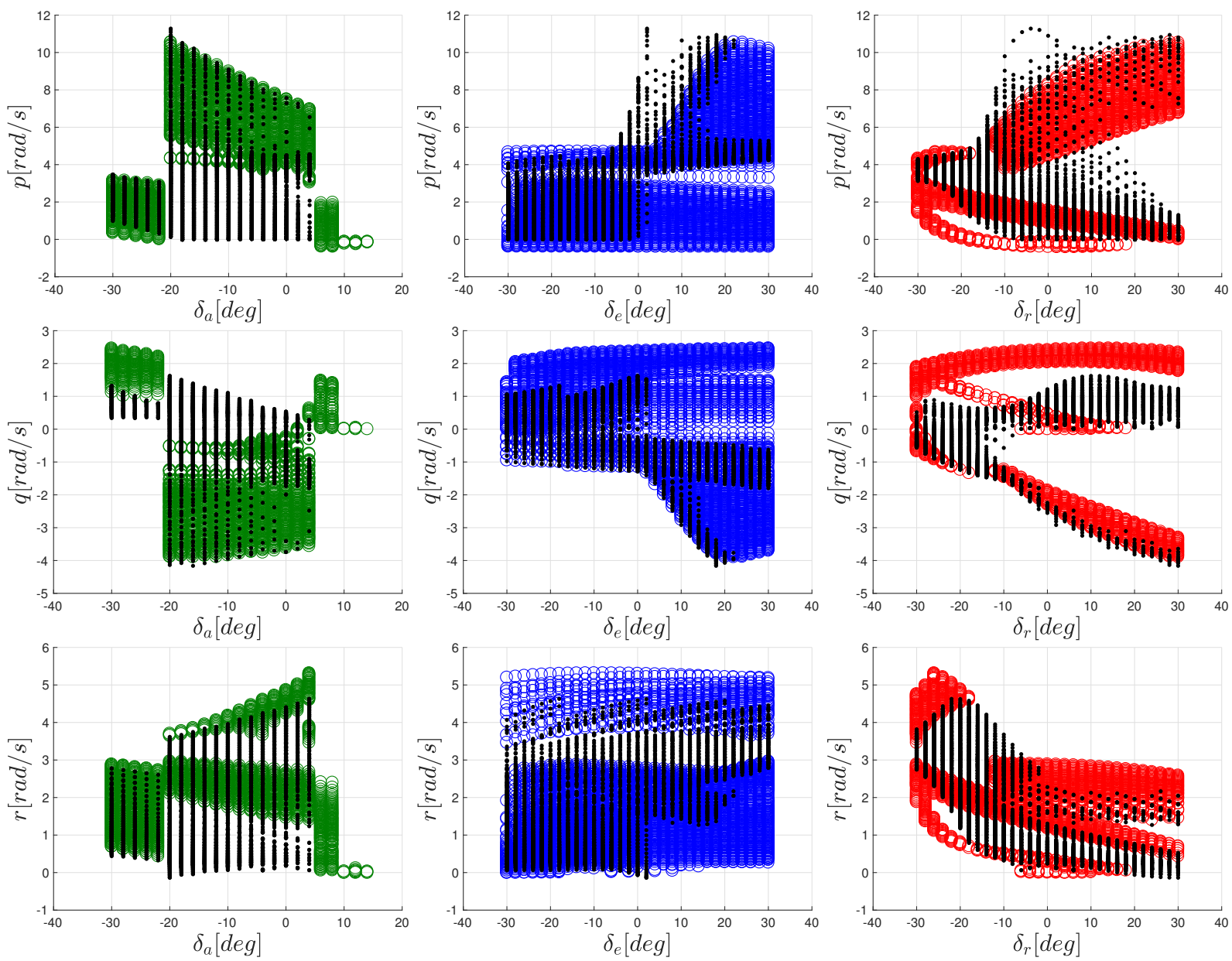

Figure C.4: Variation of the body angular velocities $p, q$ and $r$ at the stable spin equilibria, with the control inputs $\delta_{a}, \delta_{e}$ and $\delta_{r}$. The radius of the helical descent of the equilibria is restricted to $200 \mathrm{~m}$. 

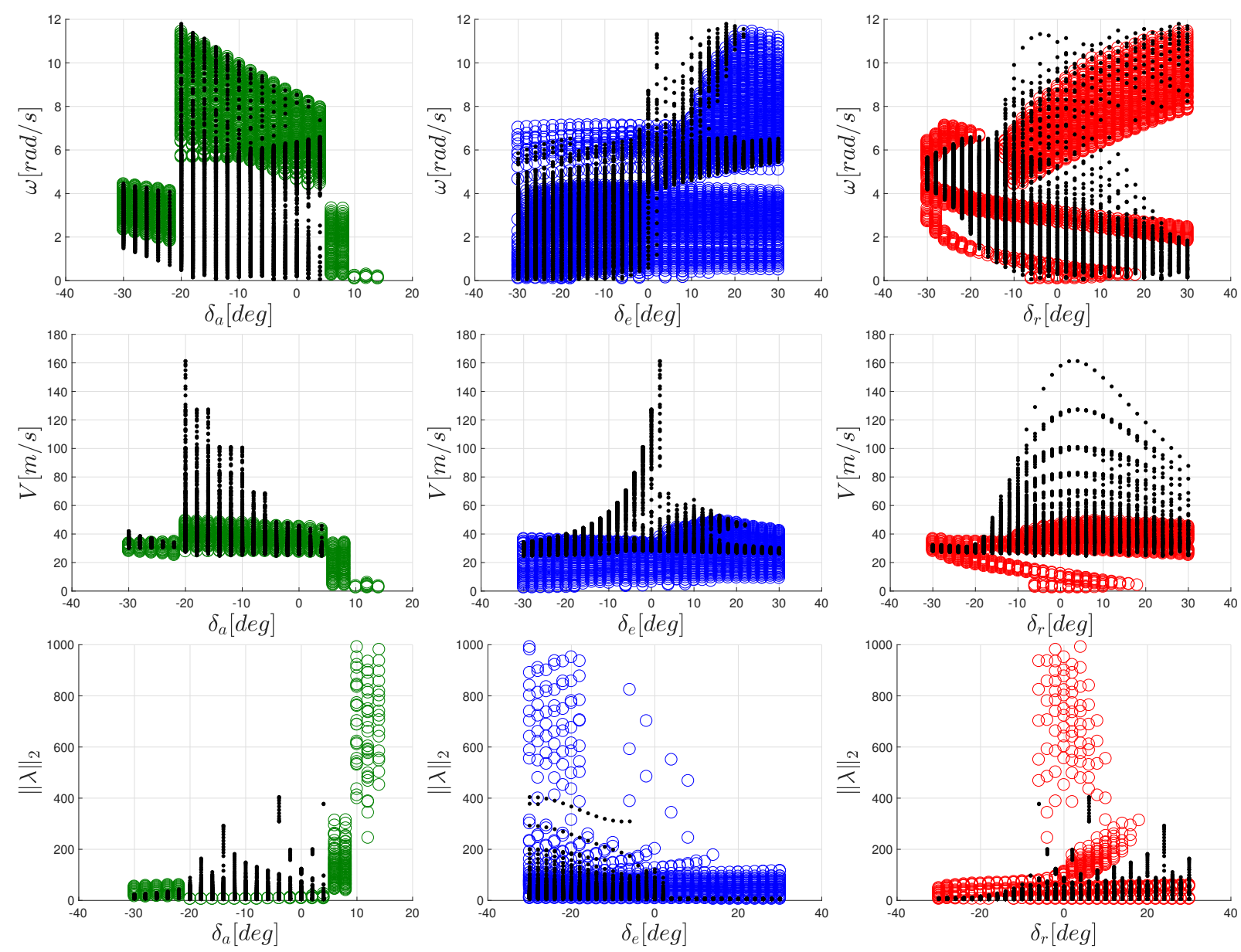

Figure C.5: Variation of the angular speed (which is equal to the yaw rate at equilibrium) $\omega$, the linear speed $V$ and the 2-norm of the eigenvalues $\|\lambda\|_{2}$ with the control inputs $\delta_{a}, \delta_{e}$ and $\delta_{r}$. The radius of the helical descent of the equilibria is restricted to $200 \mathrm{~m}$. 


\section{Bibliography}

[1] Malcolm J Abzug and E Eugene Larrabee. Airplane stability and control: a history of the technologies that made aviation possible, volume 14. Cambridge University Press, 2005.

[2] N Ananthkrishnan and Nandan K Sinha. Level flight trim and stability analysis using extended bifurcation and continuation procedure. Journal of Guidance Control and Dynamics, 24(6):1225-1227, 2001.

[3] Seth B Anderson. Historical overview of stall/spin characteristics of general aviation aircraft. Journal of Aircraft, 16(7):455-461, 1979.

[4] Anon. 2016-17 DBF Rules. AIAA, Reston, VA, 2016. http://www.aiaadbf.org/DBF-2017-Rules/.

[5] N Baghdadi, Mark H Lowenberg, and Askin T Isikveren. Analysis of flexible aircraft dynamics using bifurcation methods. Journal of Guidance Control and Dynamics, 34(3):795, 2011.

[6] P. N. Bandu. Performance, stability, dynamics, and control of airplanes. AIAA, Reston, VA, 2004.

[7] M. Bhatia, M. Patil, C. Woolsey, B. Stanford, and P. Beran. Stabilization of flapping-wing micro-air vehicles in gust environments. AIAA Journal of Guidance, Control, and Dynamics, 37(2):592-607, February 2014.

[8] William Bihrle and Billy Barnhart. Spin prediction techniques. Journal of Aircraft, 20(2):97-101, 1983.

[9] W Bihrle Jr, RS Hultberg, and W Mulcay. Rotary balance data for a typical single-engine low-wing general aviation design for an angle-of-attack range of 30 deg to $90 \mathrm{deg}, 1978$.

[10] Roger W Brockett. Finite dimensional linear systems. SIAM, 2015.

[11] Sanger M Burk Jr, James S Bowman Jr, and William L White. Spin-tunnel investigation of the spinning characteristics of typical single-engine general aviation airplane designs. i -low-wing model a: Efeects of tail configurations. NASA TP-1009, 1977. 
[12] Joseph R Chambers and Sue B Grafton. Aerodynamic characteristics of airplanes at high angles of attack. NASA TM-74097, 1977.

[13] Peter A Cummings. Continuation methods for qualitative analysis of aircraft dynamics, 2004.

[14] K. Cunningham, D. E. Cox, J. V. Foster, S. E. Riddick, and S. A. Laughter. Airstar beyond visual range UAS description and preliminary test results. In AIAA SciTech Conference, San Diego, CA, January 4-8 2016.

[15] DF Davidenko. On a new method of numerical solution of systems of nonlinear equations. In Dokl. Akad. Nauk SSSR, volume 88, pages 601-602, 1953.

[16] D. B. Doman, M. W. Oppenheimer, and D. O. Sigthorsson. Wingbeat shape modulation for flappingwing micro-air-vehicle control during hover. AIAA Journal of Guidance, Control, and Dynamics, 33(3):724-739, May 2010.

[17] Jean-Etienne T Dongmo. Aircraft stall recovery using nonlinear smooth feedback regulators with inputs constraints. In AIAA Guidance, Navigation, and Control Conference, page 6303, 2011.

[18] William Frederick Durand. Aerodynamic Theory: A General Review of Progress. Divisions AD. Julius Springer, 1934.

[19] Bernard Etkin. Dynamics of atmospheric flight. Courier Corporation, 2012.

[20] Stephen J Gill, Mark H Lowenberg, Simon A Neild, Bernd Krauskopf, Guilhem Puyou, and Etienne Coetzee. Upset dynamics of an airliner model: a nonlinear bifurcation analysis. Journal of aircraft, 2013.

[21] MG Goman, GI Zagainov, and AV Khramtsovsky. Application of bifurcation methods to nonlinear flight dynamics problems. Progress in Aerospace Sciences, 33(9-10):539-586, 1997.

[22] Mikhail G Goman, Andrew V Khramtsovsky, and Evgeny N Kolesnikov. Evaluation of aircraft performance and maneuverability by computation of attainable equilibrium sets. Journal of guidance, control, and dynamics, 31(2):329, 2008.

[23] J. A. Grauer and E. A. Morelli. A generic nonlinear aerodynamic model for aircraft. In AIAA Atmospheric Flight Mechanics Conference, Atlanta, GA, June 16-20 2014.

[24] Ph Guicheteau. Bifurcation theory in flight dynamics(an application to a real combat aircraft). Tiré à part- Office national d'études et de recherches aerospatiales, 1990.

[25] Kestrel User Guide. Kestrel autopilot (firmware version ma8. 2) \& virtual cockpit 2.4. 3, 2008. 
[26] RS Hultberg and W Mulcay. Rotary balance data for a typical single-engine general aviation design for an angle-of-attack range of $8 \mathrm{deg}$ to $90 \mathrm{deg}$. 1: Low-wing model a.[fluid flow and vortices data for general aviation aircraft to determine aerodynamic characteristics for various designs], 1980.

[27] MicroPilot Inc. Mp2028g installation and operation. Stony Mountain, Manitoba, 2005.

[28] CC Jahnke and FEC Culick. Application of bifurcation theory to the high-angle-of-attack dynamics of the f-14. Journal of Aircraft, 31(1):26-33, 1994.

[29] Thomas Jordan et al. Airstar: A uav platform for flight dynamics and control system testing. In 25th AIAA Aerodynamic Measurement Technology and Ground Testing Conference, 2006.

[30] HB Keller. Lectures on numerical methods in bifurcation problems. Applied Mathematics, 217:50, 1987.

[31] Herbert B Keller. Numerical solution of bifurcation and nonlinear eigenvalue ploblems. Applications of bifurcation theory, pages 359-384, 1977.

[32] D. A. Kennedy. Spins: Will your aircraft recover? Sport Aviation, pages 18-20, February 1975.

[33] B. Kinzig. Global hawk systems engineering case study. Technical report, Air Force Institute of Technology: Air Force Center for Systems Engineering, Wright-Patterson AFB, Ohio, 2010.

[34] RW Klopfenstein. Zeros of nonlinear functions. Journal of the ACM (JACM), 8(3):366-373, 1961.

[35] Yuri A Kuznetsov. Elements of applied bifurcation theory, volume 112. Springer Science \& Business Media, 2013.

[36] Harry G Kwatny, Jean-Etienne T Dongmo, Bor-Chin Chang, Gaurav Bajpai, Murat Yasar, and Christine Belcastro. Nonlinear analysis of aircraft loss of control. Journal of Guidance, Control, and Dynamics, 2013.

[37] FBJ Macmillen and JMT Thompson. Bifurcation analysis in the flight dynamics design process? a view from the aircraft industry. Philosophical Transactions-Royal Society of London series, A Mathematical, Physical and Engineering Sciences, pages 2321-2333, 1998.

[38] Raman K Mehra and James V Carroll. Global stability and control analysis of aircraft at high anglesof-attack. Technical report, Scientific Systems Inc, Cambridge - MA, 1979.

[39] Austin Matthew Murch. Aerodynamic modeling of post-stall and spin dynamics of large transport airplanes. $\mathrm{PhD}$ thesis, Georgia Institute of Technology, 2007.

[40] M. W. Oppenheimer, D. B. Doman, and D. O. Sigthorsson. Dynamics and control of a biomimetic vehicle using biased wingbeat forcing functions. AIAA Journal of Guidance, Control, and Dynamics, 34(1):204-217, January 2011. 
[41] A. A. Paranjape, S. J. Gill, N. Ananthkrishnan, and M. H. Lowenberg. Spin susceptibility of the nasa generic transport model. In AIAA Atmospheric Flight Mechanics (AFM) Conference, 2013.

[42] AA Paranjape and Narayan Ananthkrishnan. Analytical criterion for aircraft spin susceptibility. Journal of Aircraft, 47(5):1804-1807, 2010.

[43] Aditya Paranjape, Nandan Kumar Sinha, and Narayan Ananthkrishnan. Use of bifurcation and continuation methods for aircraft trim and stability analysis-a state-of-the-art. Journal of Aerospace Sciences and Technologies, 60(2):85, 2008.

[44] Wilson J Rugh. Linear system theory, volume 2. prentice hall Upper Saddle River, NJ, 1996.

[45] Nandan K Sinha and N Ananthkrishnan. Elementary flight dynamics with an introduction to bifurcation and continuation methods. CRC Press, 2013.

[46] R. S. Stansbury, C. W. Tanis, and T. A. Wilson. A technology survey of emergency recovery and flight termination systems for UAS. In AIAA Infotech@Aerospace Conference and AIAA Unmanned... Unlimited Conference, Seattle, WA, April 6-9 2009.

[47] MB Tischler and JB Barlow. Determination of the spin and recovery characteristics of a general aviation design. Journal of Aircraft, 18(4):238-244, 1981.

[48] B Vaglienti, R Hoag, and M Niculescu. Piccolo system users guide, cloud cap technology, 2008.

[49] Patroclos J Yangos and John P Yangos. Spin: angles and inertial moments. The Aeronautical Journal, 85(846):270-276, 1981. 